\title{
Multistep Hybrid Iterations for Systems of Generalized Equilibria with Constraints of Several Problems
}

\author{
Lu-Chuan Ceng, ${ }^{1}$ Cheng-Wen Liao, ${ }^{2}$ Chin-Tzong Pang, ${ }^{3}$ and Ching-Feng Wen ${ }^{4}$ \\ ${ }^{1}$ Scientific Computing Key Laboratory of Shanghai Universities, Department of Mathematics, Shanghai Normal University, \\ Shanghai 200234, China \\ ${ }^{2}$ Department of Food and Beverage Management, Vanung University, Chung-Li 320061, Taiwan \\ ${ }^{3}$ Department of Information Management, and Innovation Center for Big Data and Digital Convergence, Yuan Ze University, \\ Chung-Li 32003, Taiwan \\ ${ }^{4}$ Center for Fundamental Science, Kaohsiung Medical University, Kaohsiung 807, Taiwan
}

Correspondence should be addressed to Chin-Tzong Pang; imctpang@saturn.yzu.edu.tw

Received 24 January 2014; Accepted 11 February 2014; Published 8 May 2014

Academic Editor: Jen-Chih Yao

Copyright (c) $2014 \mathrm{Lu}$-Chuan Ceng et al. This is an open access article distributed under the Creative Commons Attribution License, which permits unrestricted use, distribution, and reproduction in any medium, provided the original work is properly cited.

\begin{abstract}
We first introduce and analyze one multistep iterative algorithm by hybrid shrinking projection method for finding a solution of the system of generalized equilibria with constraints of several problems: the generalized mixed equilibrium problem, finitely many variational inclusions, the minimization problem for a convex and continuously Fréchet differentiable functional, and the fixedpoint problem of an asymptotically strict pseudocontractive mapping in the intermediate sense in a real Hilbert space. We prove strong convergence theorem for the iterative algorithm under suitable conditions. On the other hand, we also propose another multistep iterative algorithm involving no shrinking projection method and derive its weak convergence under mild assumptions.
\end{abstract}

\section{Introduction}

Let $C$ be a nonempty closed convex subset of a real Hilbert space $H$ and $P_{C}$ the metric projection of $H$ onto $C$. Let $S$ : $C \rightarrow H$ be a nonlinear mapping on $C$. We denote by Fix $(S)$ the set of fixed points of $S$ and by $\mathbf{R}$ the set of all real numbers. A mapping $V$ is called strongly positive on $H$ if there exists a constant $\bar{\gamma}>0$ such that

$$
\langle V x, x\rangle \geq \bar{\gamma}\|x\|^{2}, \quad \forall x \in H .
$$

A mapping $S: C \rightarrow H$ is called $L$-Lipschitz continuous if there exists a constant $L \geq 0$ such that

$$
\|S x-S y\| \leq L\|x-y\|, \quad \forall x, y \in C .
$$

In particular, if $L=1$, then $A$ is called a nonexpansive mapping; if $L \in[0,1)$, then $S$ is called a contraction.

Let $A: C \rightarrow H$ be a nonlinear mapping on $C$. We consider the following variational inequality problem (VIP): find a point $x \in C$ such that

$$
\langle A x, y-x\rangle \geq 0, \quad \forall y \in C .
$$

The solution set of VIP (3) is denoted by VI $(C, A)$. We remark that VIP (3) was first discussed by Lions [1].

Let $\varphi: C \rightarrow \mathbf{R}$ be a real-valued function, $A: H \rightarrow H$ a nonlinear mapping, and $\Theta: C \times C \rightarrow \mathbf{R}$ a bifunction. In 2008, Peng and Yao [2] introduced the following generalized mixed equilibrium problem (GMEP) of finding $x \in C$ such that

$$
\Theta(x, y)+\varphi(y)-\varphi(x)+\langle A x, y-x\rangle \geq 0, \quad \forall y \in C .
$$

We denote the set of solutions of GMEP (4) by GMEP $(\Theta, \varphi, A)$. The GMEP $(4)$ is very general which includes, as special cases, the generalized equilibrium problem [3], the mixed equilibrium problem [4], and the equilibrium problem $[5,6]$.

In [2], it is assumed that $\Theta: C \times C \rightarrow \mathbf{R}$ is a bifunction satisfying conditions ( $\mathrm{H} 1)-(\mathrm{H} 4)$ and $\varphi: C \rightarrow \mathbf{R}$ is a lower semicontinuous and convex function with restriction (H5), where

(H1) $\Theta(x, x)=0$ for all $x \in C$; 
(H2) $\Theta$ is monotone; that is, $\Theta(x, y)+\Theta(y, x) \leq 0$ for any $x, y \in C$;

(H3) $\Theta$ is upper hemicontinuous; that is, for each $x, y, z \in C$,

$$
\limsup _{t \rightarrow 0^{+}} \Theta(t z+(1-t) x, y) \leq \Theta(x, y)
$$

(H4) $\Theta(x, \cdot)$ is convex and lower semicontinuous for each $x \in C$;

(H5) for each $x \in H$ and $r>0$, there exists a bounded subset $D_{x} \subset C$ and $y_{x} \in C$ such that for any $z \in C \backslash D_{x}$,

$$
\Theta\left(z, y_{x}\right)+\varphi\left(y_{x}\right)-\varphi(z)+\frac{1}{r}\left\langle y_{x}-z, z-x\right\rangle<0 .
$$

Given a positive number $r>0$. Let $S_{r}^{(\Theta, \varphi)}: H \rightarrow C$ be the solution set of the auxiliary mixed equilibrium problem; that is, for each $x \in H$,

$$
\begin{aligned}
& S_{r}^{(\Theta, \varphi)}(x) \\
& :=\{y \in C: \Theta(y, z)+\varphi(z)-\varphi(y) \\
& \left.\quad+\frac{1}{r}\left\langle K^{\prime}(y)-K^{\prime}(x), z-y\right\rangle \geq 0, \forall z \in C\right\} .
\end{aligned}
$$

In particular, whenever $K(x)=(1 / 2)\|x\|^{2}, \forall x \in H, S_{r}^{(\Theta, \varphi)}(x)$ is rewritten as $T_{r}^{(\Theta, \varphi)}(x)$. Further, if $\varphi=0$ additionally, then $T_{r}^{(\Theta, \varphi)}(x)$ is rewritten as $T_{r}^{\Theta}(x)$.

Let $\Theta_{1}, \Theta_{2}: C \times C \stackrel{\mathrm{R}}{\rightarrow}$ be two bifunctions satisfying conditions (H1)-(H4), and let $A_{1}, A_{2}: C \rightarrow H$ be two nonlinear mappings. Consider the following system of generalized equilibrium problems (SGEP): find $\left(x^{*}, y^{*}\right) \in$ $C \times C$ such that

$$
\begin{array}{r}
\Theta_{1}\left(x^{*}, x\right)+\left\langle A_{1} y^{*}, x-x^{*}\right\rangle+\frac{1}{v_{1}}\left\langle x^{*}-y^{*}, x-x^{*}\right\rangle \geq 0, \\
\forall x \in C, \\
\Theta_{2}\left(y^{*}, y\right)+\left\langle A_{2} x^{*}, y-y^{*}\right\rangle+\frac{1}{v_{2}}\left\langle y^{*}-x^{*}, y-y^{*}\right\rangle \geq 0, \\
\forall y \in C,
\end{array}
$$

where $v_{1}>0$ and $\nu_{2}>0$ are two constants. It is introduced and studied in [7]. Whenever $\Theta_{1} \equiv \Theta_{2} \equiv 0$, the SGEP reduces to a system of variational inequalities, which is considered and studied in [8]. It is worth to mention that the system of variational inequalities is a tool to solve the Nash equilibrium problem for noncooperative games. In 2010, Ceng and Yao [7] transformed the SGEP into a fixed-point problem of the mapping $G=T_{v_{1}}^{\Theta_{1}}\left(I-v_{1} A_{1}\right) T_{v_{2}}^{\Theta_{2}}\left(I-v_{2} A_{2}\right)$. Here, we denote the fixed point set of $G$ by $\operatorname{SGEP}(G)$.
Let $\left\{T_{n}\right\}_{n=1}^{\infty}$ be an infinite family of nonexpansive mappings on $H$ and $\left\{\lambda_{n}\right\}_{n=1}^{\infty}$ a sequence of nonnegative numbers in $[0,1]$. For any $n \geq 1$, define a mapping $W_{n}$ on $H$ as follows:

$$
\begin{aligned}
U_{n, n+1}= & I, \\
U_{n, n} & =\lambda_{n} T_{n} U_{n, n+1}+\left(1-\lambda_{n}\right) I, \\
U_{n, n-1} & =\lambda_{n-1} T_{n-1} U_{n, n}+\left(1-\lambda_{n-1}\right) I, \\
& \vdots \\
U_{n, k}= & \lambda_{k} T_{k} U_{n, k+1}+\left(1-\lambda_{k}\right) I, \\
U_{n, k-1}= & \lambda_{k-1} T_{k-1} U_{n, k}+\left(1-\lambda_{k-1}\right) I, \\
& \vdots \\
U_{n, 2}= & \lambda_{2} T_{2} U_{n, 3}+\left(1-\lambda_{2}\right) I, \\
W_{n}= & U_{n, 1}=\lambda_{1} T_{1} U_{n, 2}+\left(1-\lambda_{1}\right) I .
\end{aligned}
$$

Such a mapping $W_{n}$ is called the $W$-mapping generated by $T_{n}, T_{n-1}, \ldots, T_{1}$ and $\lambda_{n}, \lambda_{n-1}, \ldots, \lambda_{1}$.

Let $Q: H \rightarrow H$ be a contraction and $V$ a strongly positive bounded linear operator on $H$. Assume that $\varphi: H \rightarrow$ $\mathbf{R}$ is a lower semicontinuous and convex functional, such that $\Theta, \Theta_{1}, \Theta_{2}: H \times H \rightarrow \mathbf{R}$ satisfy conditions (H1)-(H4) and that $A, A_{1}, A_{2}: H \rightarrow H$ are inverse-strongly monotone. Very recently, Ceng et al. [9] introduced the following hybrid extragradient-like iterative algorithm:

$$
\begin{aligned}
z_{n}= & S_{r_{n}}^{(\Theta, \varphi)}\left(x_{n}-r_{n} A x_{n}\right), \\
x_{n+1}= & \alpha_{n}\left(u+\gamma Q x_{n}\right)+\beta_{n} x_{n} \\
& +\left(\left(1-\beta_{n}\right) I-\alpha_{n}(I+\mu V)\right) W_{n} G z_{n}, \quad \forall n \geq 0,
\end{aligned}
$$

for finding a common solution of GMEP (4), SGEP (8), and the fixed point problem of an infinite family of nonexpansive mappings $\left\{T_{n}\right\}_{n=1}^{\infty}$ on $H$, where $\left\{r_{n}\right\} \subset(0, \infty),\left\{\alpha_{n}\right\},\left\{\beta_{n}\right\} \subset$ $(0,1), v_{k} \in\left(0,2 \zeta_{k}\right), k=1,2$, and $x_{0}, u \in H$ are given. The authors proved the strong convergence of the sequence $\left\{x_{n}\right\}$ to a point $x^{*} \in \Omega:=\cap_{n=1}^{\infty} \operatorname{Fix}\left(T_{n}\right) \cap \operatorname{GMEP}(\Theta, \varphi, A) \cap \operatorname{SGEP}(G)$ under some suitable conditions. This point $x^{*}$ also solves the following optimization problem:

$$
\min _{x \in \Omega} \frac{\mu}{2}\langle V x, x\rangle+\frac{1}{2}\|x-u\|^{2}-h(x),
$$

where $h: H \rightarrow \mathbf{R}$ is the potential function of $\gamma Q$.

Let $B$ be a single-valued mapping of $C$ into $H$ and $R$ a multivalued mapping with $D(R)=C$. Consider the following variational inclusion: find a point $x \in C$ such that

$$
0 \in B x+R x \text {. }
$$

We denote by $I(B, R)$ the solution set of the variational inclusion (11).

Let $f: C \rightarrow \mathbf{R}$ be a convex and continuously Fréchet differentiable functional. Consider the convex minimization problem (CMP) of minimizing $f$ over the constraint set $C$

$$
\operatorname{minimize}\{f(x): x \in C\} \text {, }
$$


which was studied in [10-13]. We denote by $\Gamma$ the set of minimizers of CMP (12).

Let $C$ be a nonempty subset of a normed space $X$. A mapping $S: C \rightarrow C$ is called uniformly Lipschitzian if there exists a constant $\mathscr{L}>0$ such that

$$
\left\|S^{n} x-S^{n} y\right\| \leq \mathscr{L}\|x-y\|, \quad \forall n \geq 1, \forall x, y \in C .
$$

Recently, Kim and Xu [14] introduced the concept of asymptotically $k$-strict pseudocontractive mappings in a Hilbert space as below.

Definition 1. Let $C$ be a nonempty subset of a Hilbert space $H$. A mapping $S: C \rightarrow C$ is said to be an asymptotically $k$ strict pseudocontractive mapping with sequence $\left\{\gamma_{n}\right\}$ if there exists a constant $k \in[0,1)$ and a sequence $\left\{\gamma_{n}\right\}$ in $[0, \infty)$ with $\lim _{n \rightarrow \infty} \gamma_{n}=0$ such that

$$
\begin{array}{r}
\left\|S^{n} x-S^{n} y\right\|^{2} \leq\left(1+\gamma_{n}\right)\|x-y\|^{2} \\
+k\left\|x-S^{n} x-\left(y-S^{n} y\right)\right\|^{2}, \\
\forall n \geq 1, \forall x, y \in C .
\end{array}
$$

It is important to note that every asymptotically $k$ strict pseudocontractive mapping with sequence $\left\{\gamma_{n}\right\}$ is a uniformly $\mathscr{L}$-Lipschitzian mapping with $\mathscr{L}=\sup \{(k+$ $\left.\left.\sqrt{1+(1-k) \gamma_{n}}\right) /(1+k): n \geq 1\right\}$. Subsequently, Sahu et al. [15] considered the concept of asymptotically $k$-strict pseudocontractive mappings in the intermediate sense, which are not necessarily Lipschitzian.

Definition 2. Let $C$ be a nonempty subset of a Hilbert space $H$. A mapping $S: C \rightarrow C$ is said to be an asymptotically $k$ strict pseudocontractive mapping in the intermediate sense with sequence $\left\{\gamma_{n}\right\}$ if there exist a constant $k \in[0,1)$ and a sequence $\left\{\gamma_{n}\right\}$ in $[0, \infty)$ with $\lim _{n \rightarrow \infty} \gamma_{n}=0$ such that

$$
\begin{array}{r}
\limsup _{n \rightarrow \infty} \sup _{x, y \in C}\left(\left\|S^{n} x-S^{n} y\right\|^{2}-\left(1+\gamma_{n}\right)\|x-y\|^{2}\right. \\
\left.-k\left\|x-S^{n} x-\left(y-S^{n} y\right)\right\|^{2}\right) \leq 0 .
\end{array}
$$

Put $\quad c_{n}:=\max \left\{0, \sup _{x, y \in C}\left(\left\|S^{n} x-S^{n} y\right\|^{2}-(1+\right.\right.$ $\left.\left.\left.\gamma_{n}\right)\|x-y\|^{2}-k\left\|x-S^{n} x-\left(y-S^{n} y\right)\right\|^{2}\right)\right\}$. Then $c_{n} \geq 0(\forall n \geq$ $1), c_{n} \rightarrow 0(n \rightarrow \infty)$, and there holds the relation

$$
\begin{aligned}
&\left\|S^{n} x-S^{n} y\right\|^{2} \leq\left(1+\gamma_{n}\right)\|x-y\|^{2} \\
&+k\left\|x-S^{n} x-\left(y-S^{n} y\right)\right\|^{2}+c_{n}, \\
& \forall n \geq 1, \quad \forall x, y \in C .
\end{aligned}
$$

In this paper, we first introduce and analyze one multistep iterative algorithm by hybrid shrinking projection method for finding a solution of the SGEP (8) with constraints of several problems: the GMEP (4), the CMP (12), finitely many variational inclusions, and the fixed point problem of an asymptotically strict pseudocontractive mapping in the intermediate sense in a real Hilbert space. We prove strong convergence theorem for the iterative algorithm under suitable conditions. On the other hand, we also propose another multistep iterative algorithm involving no shrinking projection method and derive its weak convergence under mild assumptions. Our results improve and extend the corresponding results in the earlier and recent literatures.

\section{Preliminaries}

Throughout this paper, we assume that $H$ is a real Hilbert space whose inner product and norm are denoted by $\langle\cdot, \cdot\rangle$ and $\|\cdot\|$, respectively. Let $C$ be a nonempty closed convex subset of $H$. We write $x_{n} \rightarrow x$ to indicate that the sequence $\left\{x_{n}\right\}$ converges weakly to $x$ and $x_{n} \rightarrow x$ to indicate that the sequence $\left\{x_{n}\right\}$ converges strongly to $x$. Moreover, we use $\omega_{w}\left(x_{n}\right)$ to denote the weak $\omega$-limit set of the sequence $\left\{x_{n}\right\}$; that is,

$$
\begin{aligned}
& \omega_{w}\left(x_{n}\right) \\
& :=\left\{x \in H: x_{n_{i}} \rightarrow x \text { for some subsequence }\left\{x_{n_{i}}\right\} \text { of }\left\{x_{n}\right\}\right\} .
\end{aligned}
$$

Recall that a mapping $A: C \rightarrow H$ is called

(i) monotone if

$$
\langle A x-A y, x-y\rangle \geq 0, \quad \forall x, y \in C ;
$$

(ii) $\eta$-strongly monotone if there exists a constant $\eta>0$ such that

$$
\langle A x-A y, x-y\rangle \geq \eta\|x-y\|^{2}, \quad \forall x, y \in C ;
$$

(iii) $\alpha$-inverse-strongly monotone if there exists a constant $\alpha>0$ such that

$$
\langle A x-A y, x-y\rangle \geq \alpha\|A x-A y\|^{2}, \quad \forall x, y \in C .
$$

It is obvious that if $A$ is $\alpha$-inverse-strongly monotone, then $A$ is monotone and $(1 / \alpha)$-Lipschitz continuous.

The metric (or nearest point) projection from $H$ onto $C$ is the mapping $P_{C}: H \rightarrow C$ which assigns to each point $x \in H$ the unique point $P_{C} x \in C$ satisfying the property

$$
\left\|x-P_{C} x\right\|=\inf _{y \in C}\|x-y\|=: d(x, C) .
$$

Some important properties of projections are gathered in the following proposition.

Proposition 3. For given $x \in H$ and $z \in C$ :

(i) $z=P_{C} x \Leftrightarrow\langle x-z, y-z\rangle \leq 0, \forall y \in C$;

(ii) $z=P_{C} x \Leftrightarrow\|x-z\|^{2} \leq\|x-y\|^{2}-\|y-z\|^{2}, \forall y \in C$;

(iii) $\left\langle P_{C} x-P_{C} y, x-y\right\rangle \geq\left\|P_{C} x-P_{C} y\right\|^{2}, \forall y \in H$.

Consequently, $P_{C}$ is nonexpansive and monotone.

If $A$ is an $\alpha$-inverse-strongly monotone mapping of $C$ into $H$, then it is obvious that $A$ is $(1 / \alpha)$-Lipschitz continuous. We also have that if $\lambda \leq 2 \alpha$, then $I-\lambda A$ is a nonexpansive mapping from $C$ to $H$. 
Definition 4. A mapping $T: H \rightarrow H$ is said to be

(a) nonexpansive if

$$
\|T x-T y\| \leq\|x-y\|, \quad \forall x, y \in H
$$

(b) firmly nonexpansive if $2 T-I$ is nonexpansive, or equivalently, if $T$ is 1 -inverse strongly monotone (1ism),

$$
\langle x-y, T x-T y\rangle \geq\|T x-T y\|^{2}, \quad \forall x, y \in H
$$

alternatively, $T$ is firmly nonexpansive if and only if $T$ can be expressed as

$$
T=\frac{1}{2}(I+S)
$$

where $S: H \rightarrow H$ is nonexpansive; projections are firmly nonexpansive.

It can be easily seen that if $T$ is nonexpansive, then $I-T$ is monotone.

Definition 5. A mapping $T: H \rightarrow H$ is said to be an averaged mapping if it can be written as the average of the identity $I$ and a nonexpansive mapping; that is,

$$
T \equiv(1-\alpha) I+\alpha S
$$

where $\alpha \in(0,1)$ and $S: H \rightarrow H$ is nonexpansive. More precisely, when the last equality holds, we say that $T$ is $\alpha$ averaged. Thus firmly nonexpansive mappings (in particular, projections) are (1/2)-averaged mappings.

Proposition 6 (see [16]). Let $T: H \rightarrow H$ be a given mapping.

(i) $T$ is nonexpansive if and only if the complement $I-T$ is $(1 / 2)-i s m$.

(ii) If $T$ is $\nu$-ism, then for $\gamma>0, \gamma T$ is $(\nu / \gamma)$-ism.

(iii) $T$ is averaged if and only if the complement $I-T$ is $\nu$ ism for some $v>1 / 2$. Indeed, for $\alpha \in(0,1), T$ is $\alpha$ averaged if and only if $I-T$ is $(1 / 2 \alpha)$-ism.

Proposition 7 (see [16]). Let $S, T, V: H \rightarrow H$ be given operators.

(i) If $T=(1-\alpha) S+\alpha V$ for some $\alpha \in(0,1)$ and if $S$ is averaged and $V$ is nonexpansive, then $T$ is averaged.

(ii) $T$ is firmly nonexpansive if and only if the complement $I-T$ is firmly nonexpansive.

(iii) If $T=(1-\alpha) S+\alpha V$ for some $\alpha \in(0,1)$ and if $S$ is firmly nonexpansive and $V$ is nonexpansive, then $T$ is averaged.

(iv) The composite of finitely many averaged mappings is averaged. That is, if each of the mappings $\left\{T_{i}\right\}_{i=1}^{N}$ is averaged, then so is the composite $T_{1}, \ldots, T_{N}$. In particular, if $T_{1}$ is $\alpha_{1}$-averaged and $T_{2}$ is $\alpha_{2}$-averaged, where $\alpha_{1}, \alpha_{2} \in(0,1)$, then the composite $T_{1} T_{2}$ is $\alpha$ averaged, where $\alpha=\alpha_{1}+\alpha_{2}-\alpha_{1} \alpha_{2}$. (v) If the mappings $\left\{T_{i}\right\}_{i=1}^{N}$ are averaged and have a common fixed point, then

$$
\bigcap_{i=1}^{N} \operatorname{Fix}\left(T_{i}\right)=\operatorname{Fix}\left(T_{1}, \ldots, T_{N}\right) .
$$

The notation $\operatorname{Fix}(T)$ denotes the set of all fixed points of the mapping $T$; that is, $\operatorname{Fix}(T)=\{x \in H: T x=x\}$.

By using the technique in [4], we can readily obtain the following elementary result.

Proposition 8 (see [9, Lemma 1 and Proposition 1]). Let $C$ be a nonempty closed convex subset of a real Hilbert space $H$ and let $\varphi: C \rightarrow \mathbf{R}$ be a lower semicontinuous and convex function. Let $\Theta: C \times C \rightarrow \mathbf{R}$ be a bifunction satisfying the conditions (H1)-(H4). Assume that

(i) $K: H \rightarrow \mathbf{R}$ is strongly convex with constant $\sigma>0$ and the function $x \mapsto\left\langle y-x, K^{\prime}(x)\right\rangle$ is weakly upper semicontinuous for each $y \in H$;

(ii) for each $x \in H$ and $r>0$, there exists a bounded subset $D_{x} \subset C$ and $y_{x} \in C$ such that for any $z \in C \backslash D_{x}$,

$\Theta\left(z, y_{x}\right)+\varphi\left(y_{x}\right)-\varphi(z)+\frac{1}{r}\left\langle K^{\prime}(z)-K^{\prime}(x), y_{x}-z\right\rangle<0$.

Then the following hold:

(a) for each $x \in H, S_{r}^{(\Theta, \varphi)}(x) \neq \emptyset$;

(b) $S_{r}^{(\Theta, \varphi)}$ is single-valued;

(c) $S_{r}^{(\Theta, \varphi)}$ is nonexpansive if $K^{\prime}$ is Lipschitz continuous with constant $v>0$ and

$$
\begin{aligned}
& \left\langle K^{\prime}\left(x_{1}\right)-K^{\prime}\left(x_{2}\right), u_{1}-u_{2}\right\rangle \\
& \quad \leq\left\langle K^{\prime}\left(u_{1}\right)-K^{\prime}\left(u_{2}\right), u_{1}-u_{2}\right\rangle, \quad \forall\left(x_{1}, x_{2}\right) \in H \times H,
\end{aligned}
$$

where $u_{i}=S_{r}^{(\Theta, \varphi)}\left(x_{i}\right)$ for $i=1,2$;

(d) for all $s, t>0$ and $x \in H$

$$
\begin{aligned}
& \left\langle K^{\prime}\left(S_{s}^{(\Theta, \varphi)} x\right)-K^{\prime}\left(S_{t}^{(\Theta, \varphi)} x\right), S_{s}^{(\Theta, \varphi)} x-S_{t}^{(\Theta, \varphi)} x\right\rangle \\
& \quad \leq \frac{s-t}{s}\left\langle K^{\prime}\left(S_{s}^{(\Theta, \varphi)} x\right)-K^{\prime}(x), S_{s}^{(\Theta, \varphi)} x-S_{t}^{(\Theta, \varphi)} x\right\rangle ;
\end{aligned}
$$

(e) $\operatorname{Fix}\left(S_{r}^{(\Theta, \varphi)}\right)=\operatorname{MEP}(\Theta, \varphi)$;

(f) $\operatorname{MEP}(\Theta, \varphi)$ is closed and convex.

Remark 9. In Proposition 6, whenever $\Theta: C \times C \rightarrow \mathbf{R}$ is a bifunction satisfying the conditions $(\mathrm{H} 1)-(\mathrm{H} 4)$ and $K(x)=$ $(1 / 2)\|x\|^{2}, \forall x \in H$, we have for any $x, y \in H$,

$$
\left\|S_{r}^{(\Theta, \varphi)} x-S_{r}^{(\Theta, \varphi)} y\right\|^{2} \leq\left\langle S_{r}^{(\Theta, \varphi)} x-S_{r}^{(\Theta, \varphi)} y, x-y\right\rangle,
$$


$\left(S_{r}^{(\Theta, \varphi)}\right.$ is firmly nonexpansive) and

$$
\begin{array}{r}
\left\|S_{s}^{(\Theta, \varphi)} x-S_{t}^{(\Theta, \varphi)} x\right\| \leq \frac{|s-t|}{s}\left\|S_{s}^{(\Theta, \varphi)} x-x\right\|, \\
\forall s, t>0, x \in H .
\end{array}
$$

In this case, $S_{r}^{(\Theta, \varphi)}$ is rewritten as $T_{r}^{(\Theta, \varphi)}$. If, in addition, $\varphi \equiv 0$, then $T_{r}^{(\Theta, \varphi)}$ is rewritten as $T_{r}^{\Theta}$.

We need some facts and tools in a real Hilbert space $H$ which are listed as lemmas below.

Lemma 10. Let $X$ be a real inner product space. Then there holds the following inequality:

$$
\|x+y\|^{2} \leq\|x\|^{2}+2\langle y, x+y\rangle, \quad \forall x, y \in X .
$$

Lemma 11. Let $A: C \rightarrow H$ be a monotone mapping. In the context of the variational inequality problem the characterization of the projection (see Proposition 3(i)) implies

$$
u \in V I(C, A) \Longleftrightarrow u=P_{C}(u-\lambda A u), \quad \lambda>0 .
$$

Lemma 12 (see [17], demiclosedness principle). Let $C$ be a nonempty closed convex subset of a real Hilbert space $H$. Let $T$ be a nonexpansive self-mapping on $C$. Then $I-T$ is demiclosed. That is, whenever $\left\{x_{n}\right\}$ is a sequence in $C$ weakly converging to some $x \in C$ and the sequence $\left\{(I-T) x_{n}\right\}$ strongly converges to some $y$, it follows that $(I-T) x=y$. Here $I$ is the identity operator of $H$.

Lemma 13 (see [18, p. 80]). Let $\left\{a_{n}\right\}_{n=1}^{\infty},\left\{b_{n}\right\}_{n=1}^{\infty}$ and $\left\{\delta_{n}\right\}_{n=1}^{\infty}$ be sequences of nonnegative real numbers satisfying the inequality

$$
a_{n+1} \leq\left(1+\delta_{n}\right) a_{n}+b_{n}, \quad \forall n \geq 1 .
$$

If $\sum_{n=1}^{\infty} \delta_{n}<\infty$ and $\sum_{n=1}^{\infty} b_{n}<\infty$, then $\lim _{n \rightarrow \infty} a_{n}$ exists. If, in addition, $\left\{a_{n}\right\}_{n=1}^{\infty}$ has a subsequence which converges to zero, then $\lim _{n \rightarrow \infty} a_{n}=0$.

Recall that a Banach space $X$ is said to satisfy the Opial condition [17] if for any given sequence $\left\{x_{n}\right\} \subset X$ which converges weakly to an element $x \in X$, there holds the inequality

$$
\limsup _{n \rightarrow \infty}\left\|x_{n}-x\right\|<\limsup _{n \rightarrow \infty}\left\|x_{n}-y\right\|, \quad \forall y \in X, y \neq x .
$$

It is well known in [17] that every Hilbert space $H$ satisfies the Opial condition.

Lemma 14 (see [19, Proposition 3.1]). Let $C$ be a nonempty closed convex subset of a real Hilbert space $H$ and let $\left\{x_{n}\right\}$ be a sequence in $H$. Suppose that

$$
\left\|x_{n+1}-p\right\|^{2} \leq\left(1+\lambda_{n}\right)\left\|x_{n}-p\right\|^{2}+\delta_{n}, \quad \forall p \in C, n \geq 1,
$$

where $\left\{\lambda_{n}\right\}$ and $\left\{\delta_{n}\right\}$ are sequences of nonnegative real numbers such that $\sum_{n=1}^{\infty} \lambda_{n}<\infty$ and $\sum_{n=1}^{\infty} \delta_{n}<\infty$. Then $\left\{P_{C} x_{n}\right\}$ converges strongly in $C$.
Lemma 15 (see [20]). Let $C$ be a closed convex subset of a real Hilbert space $H$. Let $\left\{x_{n}\right\}$ be a sequence in $H$ and $u \in H$. Let $q=P_{C} u$. If $\left\{x_{n}\right\}$ is such that $\omega_{w}\left(x_{n}\right) \subset C$ and satisfies the condition

$$
\left\|x_{n}-u\right\| \leq\|u-q\|, \quad \forall n,
$$

then $x_{n} \rightarrow$ qas $n \rightarrow \infty$.

Lemma 16. Let $H$ be a real Hilbert space. Then the following hold:

(a) $\|x-y\|^{2}=\|x\|^{2}-\|y\|^{2}-2\langle x-y, y\rangle$ for all $x, y \in H$;

(b) $\|\lambda x+\mu y\|^{2}=\lambda\|x\|^{2}+\mu\|y\|^{2}-\lambda \mu\|x-y\|^{2}$ for all $x, y \in$ $H$ and $\lambda, \mu \in[0,1]$ with $\lambda+\mu=1$;

(c) if $\left\{x_{n}\right\}$ is a sequence in $H$ such that $x_{n} \rightarrow x$, it follows that

$\limsup _{n \rightarrow \infty}\left\|x_{n}-y\right\|^{2}=\limsup _{n \rightarrow \infty}\left\|x_{n}-x\right\|^{2}+\|x-y\|^{2}, \quad \forall y \in H$.

Assume that $R: D(R) \subset H \rightarrow 2^{H}$ is a maximal monotone mapping. Then, for $\lambda>0$, associated with $R$, the resolvent operator $J_{R, \lambda}$ can be defined as

$$
J_{R, \lambda} x=(I+\lambda R)^{-1} x, \quad \forall x \in H .
$$

In terms of Huang [21] (see also [22]), there holds the following property for the resolvent operator $J_{R, \lambda}: H \rightarrow \overline{D(R)}$.

Lemma 17. $J_{R, \lambda}$ is single-valued and firmly nonexpansive; that is,

$$
\left\langle J_{R, \lambda} x-J_{R, \lambda} y, x-y\right\rangle \geq\left\|J_{R, \lambda} x-J_{R, \lambda} y\right\|^{2}, \quad \forall x, y \in H .
$$

Consequently, $J_{R, \lambda}$ is nonexpansive and monotone.

Lemma 18 (see [23]). Let $R$ be a maximal monotone mapping with $D(R)=C$. Then for any given $\lambda>0, u \in C$ is a solution of problem (11) if and only if $u \in C$ satisfies

$$
u=J_{R, \lambda}(u-\lambda B u) .
$$

Lemma 19 (see [22]). Let $R$ be a maximal monotone mapping with $D(R)=C$ and let $B: C \rightarrow H$ be a strongly monotone, continuous, and single-valued mapping. Then for each $z \in H$, the equation $z \in(B+\lambda R) x$ has a unique solution $x_{\lambda}$ for $\lambda>0$.

Lemma 20 (see [23]). Let $R$ be a maximal monotone mapping with $D(R)=C$ and $B: C \rightarrow H$ a monotone, continuous, and single-valued mapping. Then $(I+\lambda(R+B)) C=H$ for each $\lambda>0$. In this case, $R+B$ is maximal monotone.

Lemma 21 (see [22, Lemma 2.5]). Let $H$ be a real Hilbert space. Given a nonempty closed convex subset of $H$ and points $x, y, z \in H$ and given also a real number $a \in \mathbf{R}$, the set

$$
\left\{v \in C:\|y-v\|^{2} \leq\|x-v\|^{2}+\langle z, v\rangle+a\right\}
$$

is convex (and closed). 
Recall that a set-valued mapping $T: D(T) \subset H \rightarrow 2^{H}$ is called monotone if for all $x, y \in D(T), f \in T x$ and $g \in T y$ imply

$$
\langle f-g, x-y\rangle \geq 0
$$

A set-valued mapping $T$ is called maximal monotone if $T$ is monotone and $(I+\lambda T) D(T)=H$ for each $\lambda>0$, where $I$ is the identity mapping of $H$. We denote by $G(T)$ the graph of $T$. It is known that a monotone mapping $T$ is maximal if and only if, for $(x, f) \in H \times H,\langle f-g, x-y\rangle \geq 0$ for every $(y, g) \in G(T)$ implies $f \in T x$. Let $A: C \rightarrow H$ be a monotone, $k$-Lipschitzcontinuous mapping and let $N_{C} v$ be the normal cone to $C$ at $v \in C$; that is,

$$
N_{C} v=\{w \in H:\langle v-u, w\rangle \geq 0, \forall u \in C\} .
$$

Define

$$
T v= \begin{cases}A v+N_{C} v, & \text { if } v \in C, \\ \emptyset, & \text { if } v \notin C .\end{cases}
$$

Then, $T$ is maximal monotone and $0 \in T v$ if and only if $v \in$ $\operatorname{VI}(C, A)$; see [24].

Lemma 22 (see [15, Lemma 2.6]). Let $C$ be a nonempty subset of a Hilbert space $H$ and $S: C \rightarrow C$ an asymptotically $k$-strict pseudocontractive mapping in the intermediate sense with sequence $\left\{\gamma_{n}\right\}$. Then

$$
\begin{aligned}
& \left\|S^{n} x-S^{n} y\right\| \\
& \leq \frac{1}{1-k}(k\|x-y\| \\
& \left.+\sqrt{\left(1+(1-k) \gamma_{n}\right)\|x-y\|^{2}+(1-k) c_{n}}\right)
\end{aligned}
$$

for all $x, y \in C$ and $n \geq 1$.

Lemma 23 (see [15, Lemma 2.7]). Let $C$ be a nonempty subset of a Hilbert space $H$ and $S: C \rightarrow C$ a uniformly continuous asymptotically $k$-strict pseudocontractive mapping in the intermediate sense with sequence $\left\{\gamma_{n}\right\}$. Let $\left\{x_{n}\right\}$ be a sequence in $C$ such that $\left\|x_{n}-x_{n+1}\right\| \rightarrow 0$ and $\left\|x_{n}-S^{n} x_{n}\right\| \rightarrow 0$ as $n \rightarrow \infty$. Then $\left\|x_{n}-S x_{n}\right\| \rightarrow 0$ as $n \rightarrow \infty$.

Lemma 24 (see [15, Proposition 3.1] demiclosedness principle). Let $C$ be a nonempty closed convex subset of a Hilbert space $H$ and $S: C \rightarrow C$ a continuous asymptotically $k$-strict pseudocontractive mapping in the intermediate sense with sequence $\left\{\gamma_{n}\right\}$. Then $I-S$ is demiclosed at zero in the sense that if $\left\{x_{n}\right\}$ is a sequence in $C$ such that $x_{n} \rightarrow x \in C$ and $\lim \sup _{m \rightarrow \infty} \lim \sup _{n \rightarrow \infty}\left\|x_{n}-S^{m} x_{n}\right\|=0$, then $(I-S) x=0$.

Lemma 25 (see [15, Proposition 3.2]). Let $C$ be a nonempty closed convex subset of a Hilbert space $H$ and $S: C \rightarrow C$ a continuous asymptotically $k$-strict pseudocontractive mapping in the intermediate sense with sequence $\left\{\gamma_{n}\right\}$ such that $\operatorname{Fix}(S) \neq \emptyset$. Then $\operatorname{Fix}(S)$ is closed and convex.

\section{Strong Convergence Theorem}

In this section, we will introduce and analyze one multistep iterative algorithm by hybrid shrinking projection method for finding a solution of the SGEP (8) with constraints of several problems: the GMEP (4), the CMP (12), finitely many variational inclusions, and the fixed point problem of an asymptotically strict pseudocontractive mapping in the intermediate sense in a real Hilbert space. We prove strong convergence theorem for the iterative algorithm under suitable conditions. This iterative algorithm is based on Korpelevich's extragradient method, strongly positive bounded linear operator approach, viscosity approximation method, averaged mapping approach to the GPA in [16], Mann-type iteration method, and shrinking projection method. The following proposition will play a key role in the proof of the main results in this paper.

Proposition CY (see [7]). Let $\Theta_{1}, \Theta_{2}: C \times C \rightarrow \mathbf{R}$ be two bifunctions satisfying conditions (H1)-(H4) and let $A_{k}$ : $C \rightarrow H$ be $\zeta_{k}$-inverse-strongly monotone for $k=1,2$. Let $\nu_{k} \in\left(0,2 \zeta_{k}\right)$ for $k=1,2$. Then, $\left(x^{*}, y^{*}\right) \in C \times C$ is a solution of SGEP (8) if and only if $x^{*}$ is a fixed point of the mapping $G: C \rightarrow C$ defined by $G=T_{v_{1}}^{\Theta_{1}}\left(I-\nu_{1} A_{1}\right) T_{v_{2}}^{\Theta_{2}}\left(I-\nu_{2} A_{2}\right)$, where $y^{*}=T_{v_{2}}^{\Theta_{2}}\left(I-v_{2} A_{2}\right) x^{*}$. Here, we denote by SGEP $(G)$ the fixed point set of $G$.

Theorem 26. Let $C$ be a nonempty closed convex subset of a real Hilbert space $H$. Let $N$ be an integer. Let $f: C \rightarrow \mathbf{R}$ be a convex functional with L-Lipschitz continuous gradient $\nabla f$. Let $\Theta, \Theta_{1}, \Theta_{2}$ be three bifunctions from $C \times C$ to $\mathbf{R}$ satisfying (H1)-(H4) and $\varphi: C \rightarrow \mathbf{R}$ a lower semicontinuous and convex functional. Let $R_{i}: C \rightarrow 2^{H}$ be a maximal monotone mapping and let $A, A_{k}: H \rightarrow H$ and $B_{i}: C \rightarrow H$ be $\zeta$-inverse strongly monotone, $\zeta_{k}$-inverse strongly monotone, and $\eta_{i}$-inverse-strongly monotone, respectively, for $k=1,2$ and $i=1,2, \ldots, N$. Let $S: C \rightarrow C$ be a uniformly continuous asymptotically $k$-strict pseudocontractive mapping in the intermediate sense for some $0 \leq k<1$ with sequence $\left\{\gamma_{n}\right\} \subset[0, \infty)$ such that $\lim _{n \rightarrow \infty} \gamma_{n}=0$ and $\left\{c_{n}\right\} \subset[0, \infty)$ such that $\lim _{n \rightarrow \infty} c_{n}=0$. Let $V$ be a $\bar{\gamma}$-strongly positive bounded linear operator and $Q: H \rightarrow H$ an l-Lipschitzian mapping with $\gamma l<\bar{\gamma}$. Assume that $\Omega:=\operatorname{GMEP}(\Theta, \varphi, A) \cap \operatorname{SGEP}(G) \cap$ $\cap_{i=1}^{N} I\left(B_{i}, R_{i}\right) \cap \operatorname{Fix}(S) \cap \Gamma$ is nonempty and bounded, where $G$ is defined as in Proposition CY. Let $\left\{r_{n}\right\}$ be a sequence in $[0,2 \zeta]$ and $\left\{\alpha_{n}\right\},\left\{\beta_{n}\right\}$, and $\left\{\delta_{n}\right\}$ sequences in $[0,1]$ such that $0<\alpha \leq \alpha_{n} \leq 1$ and $k \leq \delta_{n} \leq d<1$, and let $\nu_{k} \in\left(0,2 \zeta_{k}\right)$, $k=1,2$, and $\left\{\lambda_{i, n}\right\} \subset\left[a_{i}, b_{i}\right] \subset\left(0,2 \eta_{i}\right), \forall i \in\{1,2, \ldots, N\}$. Pick any $x_{0} \in H$ and set $C_{1}=C, x_{1}=P_{C_{1}} x_{0}$. Let $\left\{x_{n}\right\}$ be a sequence generated by the following algorithm:

$$
\begin{aligned}
u_{n}= & S_{r_{n}}^{(\Theta, \varphi)}\left(I-r_{n} A\right) x_{n}, \\
v_{n}= & J_{R_{N}, \lambda_{N, n}}\left(I-\lambda_{N, n} B_{N}\right) J_{R_{N-1}, \lambda_{N-1, n}} \\
& \quad \times\left(I-\lambda_{N-1, n} B_{N-1}\right), \ldots, J_{R_{1}, \lambda_{1, n}}\left(I-\lambda_{1, n} B_{1}\right) u_{n}, \\
& z_{n}=s_{n} \gamma Q x_{n}+\beta_{n} x_{n}+\left(\left(1-\beta_{n}\right) I-s_{n} V\right) T_{n} G v_{n},
\end{aligned}
$$




$$
\begin{aligned}
k_{n} & =\delta_{n} z_{n}+\left(1-\delta_{n}\right) S^{n} z_{n}, \\
y_{n} & =\left(1-\alpha_{n}\right) x_{n}+\alpha_{n} k_{n}, \\
C_{n+1} & =\left\{z \in C_{n}:\left\|y_{n}-z\right\|^{2} \leq\left\|x_{n}-z\right\|^{2}+\theta_{n}\right\}, \\
x_{n+1} & =P_{C_{n+1}} x_{0}, \quad \forall n \geq 1,
\end{aligned}
$$

where $P_{C}\left(I-\lambda_{n} \nabla f\right)=s_{n} I+\left(1-s_{n}\right) T_{n}$ (here $T_{n}$ is nonexpansive; $s_{n}=\left(2-\lambda_{n} L\right) / 4 \in(0,1 / 2)$ for each $\left.\lambda_{n} \in(0,2 / L)\right)$, and $\theta_{n}=\left(s_{n}+\gamma_{n}\right)\left(1+\gamma_{n}\right) \Delta_{n}+c_{n}, \Delta_{n}=\sup \left\{\left\|x_{n}-p\right\|^{2}+\right.$ $\left.\|(\gamma Q-V) p\|^{2} /(\bar{\gamma}-\gamma l): p \in \Omega\right\}<\infty$. Assume that the following conditions are satisfied:

(i) $K: H \rightarrow \mathbf{R}$ is strongly convex with constant $\sigma>0$ and its derivative $K^{\prime}$ is Lipschitz continuous with constant $\nu>0$ such that the function $x \mapsto\left\langle y-x, K^{\prime}(x)\right\rangle$ is weakly upper semicontinuous for each $y \in H$;

(ii) for each $x \in H$, there exist a bounded subset $D_{x} \subset C$ and $z_{x} \in C$ such that for any $y \notin D_{x}$,

$$
\begin{aligned}
& \Theta\left(y, z_{x}\right)+\varphi\left(z_{x}\right)-\varphi(y) \\
& \quad+\frac{1}{r}\left\langle K^{\prime}(y)-K^{\prime}(x), z_{x}-y\right\rangle<0 ;
\end{aligned}
$$

(iii) $s_{n} \in(0,1 / 2)$ for each $\lambda_{n} \in(0,2 / L)$, and $\lim _{n \rightarrow \infty} s_{n}=0$ $\left(\Leftrightarrow \lim _{n \rightarrow \infty} \lambda_{n}=2 / L\right)$;

(iv) $0<\liminf _{n \rightarrow \infty} \beta_{n} \leq \lim \sup _{n \rightarrow \infty} \beta_{n}<1$ and $0<$ $\liminf _{n \rightarrow \infty} r_{n} \leq \lim \sup _{n \rightarrow \infty} r_{n}<2 \zeta$.

Assume that $S_{r}^{(\Theta, \varphi)}$ is firmly nonexpansive. Then we have

(i) $\left\{x_{n}\right\}$ converges strongly as $\lambda_{n} \rightarrow(2 / L)\left(\Leftrightarrow s_{n} \rightarrow 0\right)$ to $x^{*}=P_{\Omega} x_{0}$;

(ii) $\left\{x_{n}\right\}$ converges strongly as $\lambda_{n} \rightarrow(2 / L)\left(\Leftrightarrow s_{n} \rightarrow 0\right)$ to $x^{*}=P_{\Omega} x_{0}$ provided that $\left\|x_{n}-z_{n}\right\|=o\left(s_{n}\right)$, which is the unique solution in $\Omega$ to the VIP

$$
\left\langle(\gamma Q-V) x^{*}, p-x^{*}\right\rangle \leq 0, \quad \forall p \in \Omega .
$$

Equivalently, $x^{*}=P_{\Omega}(I-V+\gamma Q) x^{*}$.

Proof. Since $\nabla f$ is $L$-Lipschitzian, it follows that $\nabla f$ is $1 / L$ ism; see [16]. By Proposition 6(ii) we know that for $\lambda>$ $0, \lambda \nabla f$ is $(1 / \lambda L)$-ism. So by Proposition 6(iii) we deduce that $I-\lambda \nabla f$ is $(\lambda L / 2)$-averaged. Now since the projection $P_{C}$ is $1 / 2$-averaged, it is easy to see from Proposition 7 (iv) that the composite $P_{C}(I-\lambda \nabla f)$ is $(2+\lambda L) / 4$-averaged for $\lambda \epsilon$ $(0,2 / L)$. Hence we obtain that for each $n \geq 1, P_{C}\left(I-\lambda_{n} \nabla f\right)$ is $\left(2+\lambda_{n} L\right) / 4$-averaged for each $\lambda_{n} \in(0,2 / L)$. Therefore, we can write

$$
P_{C}\left(I-\lambda_{n} \nabla f\right)=\frac{2-\lambda_{n} L}{4} I+\frac{2+\lambda_{n} L}{4} T_{n}=s_{n} I+\left(1-s_{n}\right) T_{n},
$$

where $T_{n}$ is nonexpansive and $s_{n}:=s_{n}\left(\lambda_{n}\right)=\left(2-\lambda_{n} L\right) / 4 \epsilon$ $(0,1 / 2)$ for each $\lambda_{n} \in(0,2 / L)$. It is clear that

$$
\lambda_{n} \longrightarrow \frac{2}{L} \Longleftrightarrow s_{n} \longrightarrow 0 .
$$

As $\lim _{n \rightarrow \infty} s_{n}=0,0<\liminf _{n \rightarrow \infty} \beta_{n} \leq \lim \sup _{n \rightarrow \infty} \beta_{n}<$ 1 and $0<\liminf _{n \rightarrow \infty} r_{n} \leq \lim \sup _{n \rightarrow \infty} r_{n}<2 \zeta$, we may assume, without loss of generality, that $\left\{\beta_{n}\right\} \subset[a, \widehat{a}] \subset(0,1)$, $\left\{r_{n}\right\} \subset[c, \widehat{c}] \subset(0,2 \zeta)$ and $\beta_{n}+s_{n}\|V\| \leq 1$ for all $n \geq 1$. Since $V$ is a $\bar{\gamma}$-strongly positive bounded linear operator on $H$, we know that

$$
\|V\|=\sup \{\langle V u, u\rangle: u \in H,\|u\|=1\} \geq \bar{\gamma}>\gamma l .
$$

Taking into account that $\beta_{n}+s_{n}\|V\| \leq 1$ for all $n \geq 1$, we have

$$
\begin{aligned}
\left\langle\left(\left(1-\beta_{n}\right) I-s_{n} V\right) u, u\right\rangle & =1-\beta_{n}-s_{n}\langle V u, u\rangle \\
& \geq 1-\beta_{n}-s_{n}\|V\| \\
& \geq 0 ;
\end{aligned}
$$

that is, $\left(1-\beta_{n}\right) I-s_{n} V$ is positive. It follows that

$$
\begin{aligned}
\|(1- & \left.\beta_{n}\right) I-s_{n} V \| \\
& =\sup \left\{\left\langle\left(\left(1-\beta_{n}\right) I-s_{n} V\right) u, u\right\rangle: u \in H,\|u\|=1\right\} \\
& =\sup \left\{1-\beta_{n}-s_{n}\langle V u, u\rangle: u \in H,\|u\|=1\right\} \\
& \leq 1-\beta_{n}-s_{n} \bar{\gamma} .
\end{aligned}
$$

Put

$$
\begin{aligned}
\Lambda_{n}^{i}= & J_{R_{i}, \lambda_{i, n}}\left(I-\lambda_{i, n} B_{i}\right) J_{R_{i-1}, \lambda_{i-1, n}} \\
& \times\left(I-\lambda_{i-1, n} B_{i-1}\right) \cdots J_{R_{1}, \lambda_{1, n}}\left(I-\lambda_{1, n} B_{1}\right)
\end{aligned}
$$

for all $i \in\{1,2, \ldots, N\}$, and $\Lambda_{n}^{0}=I$, where $I$ is the identity mapping on $H$. Then we have $v_{n}=\Lambda_{n}^{N} u_{n}$.

We divide the rest of the proof into several steps.

Step 1. We show that $\left\{x_{n}\right\}$ is well defined. It is obvious that $C_{n}$ is closed and convex. As the defining inequality in $C_{n}$ is equivalent to the inequality

$$
\left\langle 2\left(x_{n}-y_{n}\right), z\right\rangle \leq\left\|x_{n}\right\|^{2}-\left\|y_{n}\right\|^{2}+\theta_{n},
$$

by Lemma 21 we know that $C_{n}$ is convex for every $n \geq 1$. 
First of all, let us show that $\Omega \subset C_{n}$ for all $n \geq 1$. Suppose that $\Omega \subset C_{n}$ for some $n \geq 1$. Take $p \in \Omega$ arbitrarily. Since $p=S_{r_{n}}^{(\Theta, \varphi)}\left(p-r_{n} A p\right), A$ is $\zeta$-inverse strongly monotone and $0 \leq r_{n} \leq 2 \zeta$, we have, for any $n \geq 1$,

$$
\begin{aligned}
\left\|u_{n}-p\right\|^{2}= & \left\|S_{r_{n}}^{(\Theta, \varphi)}\left(I-r_{n} A\right) x_{n}-S_{r_{n}}^{(\Theta, \varphi)}\left(I-r_{n} A\right) p\right\|^{2} \\
\leq & \left\|\left(I-r_{n} A\right) x_{n}-\left(I-r_{n} A\right) p\right\|^{2} \\
= & \left\|\left(x_{n}-p\right)-r_{n}\left(A x_{n}-A p\right)\right\|^{2} \\
= & \left\|x_{n}-p\right\|^{2}-2 r_{n}\left\langle x_{n}-p, A x_{n}-A p\right\rangle \\
& +r_{n}^{2}\left\|A x_{n}-A p\right\|^{2} \\
\leq & \left\|x_{n}-p\right\|^{2}-2 r_{n} \zeta\left\|A x_{n}-A p\right\|^{2} \\
& +r_{n}^{2}\left\|A x_{n}-A p\right\|^{2} \\
= & \left\|x_{n}-p\right\|^{2}+r_{n}\left(r_{n}-2 \zeta\right)\left\|A x_{n}-A p\right\|^{2} \\
\leq & \left\|x_{n}-p\right\|^{2} .
\end{aligned}
$$

Since $p=J_{R_{i}, \lambda_{i, n}}\left(I-\lambda_{i, n} B_{i}\right) p, \Lambda_{n}^{i} p=p$ and $B_{i}$ is $\eta_{i}$-inverse strongly monotone, where $\lambda_{i, n} \in\left(0,2 \eta_{i}\right), i \in\{1,2, \ldots, N\}$, by Lemma 17 we deduce that for each $n \geq 1$,

$$
\begin{aligned}
\left\|v_{n}-p\right\|= & \| J_{R_{N}, \lambda_{N, n}}\left(I-\lambda_{N, n} B_{N}\right) \Lambda_{n}^{N-1} u_{n} \\
& -J_{R_{N}, \lambda_{N, n}}\left(I-\lambda_{N, n} B_{N}\right) \Lambda_{n}^{N-1} p \| \\
\leq & \left\|\left(I-\lambda_{N, n} B_{N}\right) \Lambda_{n}^{N-1} u_{n}-\left(I-\lambda_{N, n} B_{N}\right) \Lambda_{n}^{N-1} p\right\| \\
\leq & \left\|\Lambda_{n}^{N-1} u_{n}-\Lambda_{n}^{N-1} p\right\| \\
\vdots & \\
\leq & \left\|\Lambda_{n}^{0} x_{n}-\Lambda_{n}^{0} p\right\| \\
= & \left\|u_{n}-p\right\| .
\end{aligned}
$$

Combining (57) and (58), we have

$$
\left\|v_{n}-p\right\| \leq\left\|x_{n}-p\right\|
$$

Since $p=G p=T_{v_{1}}^{\Theta_{1}}\left(I-v_{1} A_{1}\right) T_{v_{2}}^{\Theta_{2}}\left(I-v_{2} A_{2}\right) p, A_{k}$ is $\zeta_{k}$ inverse-strongly monotone for $k=1,2$, and $0 \leq v_{k} \leq 2 \zeta_{k}$ for $k=1,2$, we deduce that, for any $n \geq 1$,

$$
\begin{aligned}
& \left\|G v_{n}-p\right\|^{2} \\
& =\| T_{v_{1}}^{\Theta_{1}}\left(I-v_{1} A_{1}\right) T_{v_{2}}^{\Theta_{2}}\left(I-v_{2} A_{2}\right) v_{n} \\
& -T_{\nu_{1}}^{\Theta_{1}}\left(I-v_{1} A_{1}\right) T_{v_{2}}^{\Theta_{2}}\left(I-v_{2} A_{2}\right) p \|^{2} \\
& \leq \|\left(I-v_{1} A_{1}\right) T_{v_{2}}^{\Theta_{2}}\left(I-v_{2} A_{2}\right) v_{n} \\
& \text { - }\left(I-v_{1} A_{1}\right) T_{v_{2}}^{\Theta_{2}}\left(I-v_{2} A_{2}\right) p \|^{2} \\
& =\|\left[T_{v_{2}}^{\Theta_{2}}\left(I-v_{2} A_{2}\right) v_{n}-T_{v_{2}}^{\Theta_{2}}\left(I-v_{2} A_{2}\right) p\right] \\
& -v_{1}\left[A_{1} T_{v_{2}}^{\Theta_{2}}\left(I-v_{2} A_{2}\right) v_{n}-A_{1} T_{v_{2}}^{\Theta_{2}}\left(I-v_{2} A_{2}\right) p\right] \|^{2} \\
& \leq\left\|T_{\nu_{2}}^{\Theta_{2}}\left(I-v_{2} A_{2}\right) v_{n}-T_{\nu_{2}}^{\Theta_{2}}\left(I-v_{2} A_{2}\right) p\right\|^{2}+v_{1}\left(\nu_{1}-2 \zeta_{1}\right) \\
& \times\left\|A_{1} T_{v_{2}}^{\Theta_{2}}\left(I-v_{2} A_{2}\right) v_{n}-A_{1} T_{v_{2}}^{\Theta_{2}}\left(I-v_{2} A_{2}\right) p\right\|^{2} \\
& \leq\left\|T_{v_{2}}^{\Theta_{2}}\left(I-v_{2} A_{2}\right) v_{n}-T_{v_{2}}^{\Theta_{2}}\left(I-v_{2} A_{2}\right) p\right\|^{2} \\
& \leq\left\|\left(I-v_{2} A_{2}\right) v_{n}-\left(\mathrm{I}-v_{2} A_{2}\right) p\right\|^{2} \\
& =\left\|\left(v_{n}-p\right)-v_{2}\left(A_{2} v_{n}-A_{2} p\right)\right\|^{2} \\
& \leq\left\|v_{n}-p\right\|^{2}+v_{2}\left(v_{2}-2 \zeta_{2}\right)\left\|A_{2} v_{n}-A_{2} p\right\|^{2} \\
& \leq\left\|v_{n}-p\right\|^{2} \text {. }
\end{aligned}
$$

(This shows that $G$ is nonexpansive.) Also, from (47), (54), (59), and (60), it follows that

$$
\begin{aligned}
& \left\|z_{n}-p\right\| \\
& =\| \beta_{n}\left(x_{n}-p\right)+s_{n} \gamma\left(Q x_{n}-Q p\right)+\left[\left(1-\beta_{n}\right) I-s_{n} V\right] \\
& \quad \times\left(T_{n} G v_{n}-p\right)+s_{n}(\gamma Q-V) p \| \\
& \leq \beta_{n}\left\|x_{n}-p\right\|+s_{n} \gamma\left\|Q x_{n}-Q p\right\| \\
& \quad+\left\|\left[\left(1-\beta_{n}\right) I-s_{n} V\right]\left(T_{n} G v_{n}-p\right)\right\|+s_{n}\|(\gamma Q-V) p\| \\
& \leq \beta_{n}\left\|x_{n}-p\right\|+s_{n} \gamma l\left\|x_{n}-p\right\|+\left(1-\beta_{n}-s_{n} \bar{\gamma}\right) \\
& \quad \times\left\|T_{n} G v_{n}-p\right\|+s_{n}\|(\gamma Q-V) p\| \\
& \leq \beta_{n}\left\|x_{n}-p\right\|+s_{n} \gamma l\left\|x_{n}-p\right\| \\
& \quad+\left(1-\beta_{n}-s_{n} \bar{\gamma}\right)\left\|G v_{n}-p\right\|+s_{n}\|(\gamma Q-V) p\| \\
& =\left(\beta_{n}+s_{n} \gamma l\right)\left\|x_{n}-p\right\|+\left(1-\beta_{n}-s_{n} \bar{\gamma}\right)\left\|G v_{n}-p\right\|
\end{aligned}
$$




$$
\begin{aligned}
& +s_{n}\|(\gamma Q-V) p\| \\
\leq & \left(\beta_{n}+s_{n} \gamma l\right)\left\|x_{n}-p\right\|+\left(1-\beta_{n}-s_{n} \bar{\gamma}\right)\left\|v_{n}-p\right\| \\
& +s_{n}\|(\gamma Q-V) p\| \\
\leq & \left(\beta_{n}+s_{n} \gamma l\right)\left\|x_{n}-p\right\|+\left(1-\beta_{n}-s_{n} \bar{\gamma}\right)\left\|x_{n}-p\right\| \\
& +s_{n}\|(\gamma Q-V) p\| \\
= & \left(1-s_{n}(\bar{\gamma}-\gamma l)\right)\left\|x_{n}-p\right\|+s_{n}\|(\gamma Q-V) p\| \\
= & \left(1-s_{n}(\bar{\gamma}-\gamma l)\right)\left\|x_{n}-p\right\|+s_{n}(\bar{\gamma}-\gamma l) \frac{\|(\gamma Q-V) p\|}{\bar{\gamma}-\gamma l},
\end{aligned}
$$

which hence yields

$$
\begin{aligned}
& \left\|z_{n}-p\right\|^{2} \\
& \leq\left(1-s_{n}(\bar{\gamma}-\gamma l)\right)\left\|x_{n}-p\right\|^{2}+s_{n}(\bar{\gamma}-\gamma l) \frac{\|(\gamma Q-V) p\|^{2}}{(\bar{\gamma}-\gamma l)^{2}} \\
& \leq\left\|x_{n}-p\right\|^{2}+s_{n} \frac{\|(\gamma Q-V) p\|^{2}}{\bar{\gamma}-\gamma l} .
\end{aligned}
$$

By Lemma 16(b), we deduce from (47) and (62) that

$$
\begin{aligned}
&\left\|k_{n}-p\right\|^{2} \\
&=\left\|\delta_{n}\left(z_{n}-p\right)+\left(1-\delta_{n}\right)\left(S^{n} z_{n}-p\right)\right\|^{2} \\
&=\delta_{n}\left\|z_{n}-p\right\|^{2}+\left(1-\delta_{n}\right)\left\|S^{n} z_{n}-p\right\|^{2} \\
& \quad-\delta_{n}\left(1-\delta_{n}\right)\left\|z_{n}-S^{n} z_{n}\right\|^{2} \\
& \leq \delta_{n}\left\|z_{n}-p\right\|^{2}+\left(1-\delta_{n}\right) \\
& \times\left[\left(1+\gamma_{n}\right)\left\|z_{n}-p\right\|^{2}+k\left\|z_{n}-S^{n} z_{n}\right\|^{2}+c_{n}\right] \\
&-\delta_{n}\left(1-\delta_{n}\right)\left\|z_{n}-S^{n} z_{n}\right\|^{2} \\
&= {\left[1+\gamma_{n}\left(1-\delta_{n}\right)\right]\left\|z_{n}-p\right\|^{2} } \\
&+\left(1-\delta_{n}\right)\left(k-\delta_{n}\right)\left\|z_{n}-S^{n} z_{n}\right\|^{2}+\left(1-\delta_{n}\right) c_{n} \\
& \leq\left(1+\gamma_{n}\right)\left\|z_{n}-p\right\|^{2}+\left(1-\delta_{n}\right)\left(k-\delta_{n}\right) \\
& \times\left\|z_{n}-S^{n} z_{n}\right\|^{2}+c_{n} \\
& \leq\left(1+\gamma_{n}\right)\left\|z_{n}-p\right\|^{2}+c_{n} \\
& \leq\left(1+\gamma_{n}\right)\left(\left\|x_{n}-p\right\|^{2}+s_{n} \frac{\|(\gamma Q-V) p\|^{2}}{\bar{\gamma}-\gamma l}\right)+c_{n} .
\end{aligned}
$$

So, from (47) and (63) we get

$$
\begin{aligned}
\| y_{n}- & p \|^{2} \\
= & \left\|\left(1-\alpha_{n}\right)\left(x_{n}-p\right)+\alpha_{n}\left(k_{n}-p\right)\right\|^{2} \\
\leq & \left(1-\alpha_{n}\right)\left\|x_{n}-p\right\|^{2}+\alpha_{n}\left\|k_{n}-p\right\|^{2} \\
\leq & \left(1-\alpha_{n}\right)\left\|x_{n}-p\right\|^{2} \\
& +\alpha_{n}\left[\left(1+\gamma_{n}\right)\right. \\
& \left.\quad \times\left(\left\|x_{n}-p\right\|^{2}+s_{n} \frac{\|(\gamma Q-V) p\|^{2}}{\bar{\gamma}-\gamma l}\right)+c_{n}\right] \\
\leq & \left(1+\gamma_{n}\right)\left(\left\|x_{n}-p\right\|^{2}+s_{n} \frac{\|(\gamma Q-V) p\|^{2}}{\bar{\gamma}-\gamma l}\right)+c_{n} \\
= & \left\|x_{n}-p\right\|^{2}+\gamma_{n}\left\|x_{n}-p\right\|^{2} \\
= & \left\|x_{n}-p\right\|^{2}+\left(s_{n}+\gamma_{n}\right)\left(1+\gamma_{n}\right) \Delta_{n}+c_{n} \\
& +\left(1+\theta_{n},\right. \\
& +\left(s_{n}+\gamma_{n}\right)\left(1+s_{n} \frac{\|(\gamma Q-V) p\|^{2}}{\bar{\gamma}-\gamma l}+c_{n}\right. \\
\leq & \left\|x_{n}-p\right\|^{2}+\gamma_{n}\left(1+\gamma_{n}\right)\left\|x_{n}-p\right\|^{2} \\
& +s_{n}\left(1+\gamma_{n}\right) \frac{\|(\gamma Q-V) p\|^{2}}{\bar{\gamma}-\gamma l}+c_{n} \\
\leq & \left\|x_{n}-p\right\|^{2}+\left(\gamma_{n}+s_{n}\right)\left(1+\gamma_{n}\right)\left\|x_{n}-p\right\|^{2} \\
& \\
& \\
& \\
&
\end{aligned}
$$

where $\theta_{n}=\left(s_{n}+\gamma_{n}\right)\left(1+\gamma_{n}\right) \Delta_{n}+c_{n}$ and $\Delta_{n}=\sup \left\{\left\|x_{n}-p\right\|^{2}+\right.$ $\left.\|(\gamma Q-V) p\|^{2} /(\bar{\gamma}-\gamma l): p \in \Omega\right\}<\infty$. Hence $p \in C_{n+1}$. This implies that $\Omega \subset C_{n}$ for all $n \geq 1$. Therefore, $\left\{x_{n}\right\}$ is well defined.

Step 2. We prove that $\left\|x_{n}-k_{n}\right\| \rightarrow 0,\left\|x_{n}-z_{n}\right\| \rightarrow 0$ and $\left\|S^{n} z_{n}-z_{n}\right\| \rightarrow 0$ as $n \rightarrow \infty$. 
Indeed, let $x^{*}=P_{\Omega} x_{0}$. From $x_{n}=P_{C_{n}} x_{0}$ and $x^{*} \in \Omega \subset$ $C_{n}$, we obtain

$$
\left\|x_{n}-x_{0}\right\| \leq\left\|x^{*}-x_{0}\right\|
$$

This implies that $\left\{x_{n}\right\}$ is bounded and hence $\left\{u_{n}\right\},\left\{v_{n}\right\},\left\{z_{n}\right\}$, $\left\{k_{n}\right\}$, and $\left\{y_{n}\right\}$ are also bounded. Since $x_{n+1} \in C_{n+1} \subset C_{n}$ and $x_{n}=P_{C_{n}} x_{0}$, we have

$$
\left\|x_{n}-x_{0}\right\| \leq\left\|x_{n+1}-x_{0}\right\|, \quad \forall n \geq 1
$$

Therefore $\lim _{n \rightarrow \infty}\left\|x_{n}-x_{0}\right\|$ exists. From $x_{n}=P_{C_{n}} x_{0}, x_{n+1} \in$ $C_{n+1} \subset C_{n}$, by Proposition 3(ii) we obtain

$$
\left\|x_{n+1}-x_{n}\right\|^{2} \leq\left\|x_{0}-x_{n+1}\right\|^{2}-\left\|x_{0}-x_{n}\right\|^{2}
$$

which implies

$$
\lim _{n \rightarrow \infty}\left\|x_{n+1}-x_{n}\right\|=0
$$

It follows from $x_{n+1} \in C_{n+1}$ that $\left\|y_{n}-x_{n+1}\right\|^{2} \leq$ $\left\|x_{n}-x_{n+1}\right\|^{2}+\theta_{n}$, and hence

$$
\begin{aligned}
\left\|x_{n}-y_{n}\right\|^{2} & \leq 2\left(\left\|x_{n}-x_{n+1}\right\|^{2}+\left\|x_{n+1}-y_{n}\right\|^{2}\right) \\
& \leq 2\left(\left\|x_{n}-x_{n+1}\right\|^{2}+\left\|x_{n}-x_{n+1}\right\|^{2}+\theta_{n}\right) \\
& =2\left(2\left\|x_{n}-x_{n+1}\right\|^{2}+\theta_{n}\right) .
\end{aligned}
$$

From (68) and $\lim _{n \rightarrow \infty} \theta_{n}=0$, we have

$$
\lim _{n \rightarrow \infty}\left\|x_{n}-y_{n}\right\|=0
$$

Since $y_{n}-x_{n}=\alpha_{n}\left(k_{n}-x_{n}\right)$ and $0<\alpha \leq \alpha_{n} \leq 1$, we have

$$
\alpha\left\|k_{n}-x_{n}\right\| \leq \alpha_{n}\left\|k_{n}-x_{n}\right\|=\left\|y_{n}-x_{n}\right\|,
$$

which immediately leads to

$$
\lim _{n \rightarrow \infty}\left\|k_{n}-x_{n}\right\|=0
$$

Also, utilizing Lemmas 10 and 16(b) we obtain from (47), (59), (60), and (63) that

$$
\begin{aligned}
& \left\|z_{n}-p\right\|^{2} \\
& =\left\|\beta_{n} x_{n}+s_{n} \gamma Q x_{n}+\left[\left(1-\beta_{n}\right) I-s_{n} V\right] T_{n} G v_{n}-p\right\|^{2} \\
& =\| \beta_{n}\left(x_{n}-p\right)+\left(1-\beta_{n}\right)\left(T_{n} G v_{n}-p\right) \\
& +s_{n}\left(\gamma Q x_{n}-V T_{n} G v_{n}\right) \|^{2} \\
& \leq\left\|\beta_{n}\left(x_{n}-p\right)+\left(1-\beta_{n}\right)\left(T_{n} G v_{n}-p\right)\right\|^{2} \\
& +2 s_{n}\left\langle\left(\gamma Q x_{n}-V T_{n} G v_{n}\right), z_{n}-p\right\rangle \\
& =\beta_{n}\left\|x_{n}-p\right\|^{2}+\left(1-\beta_{n}\right)\left\|T_{n} G v_{n}-p\right\|^{2} \\
& -\beta_{n}\left(1-\beta_{n}\right)\left\|x_{n}-T_{n} G v_{n}\right\|^{2} \\
& +2 s_{n}\left\langle\left(\gamma Q x_{n}-V T_{n} G v_{n}\right), z_{n}-p\right\rangle \\
& \leq \beta_{n}\left\|x_{n}-p\right\|^{2}+\left(1-\beta_{n}\right)\left\|G v_{n}-p\right\|^{2} \\
& -\beta_{n}\left(1-\beta_{n}\right)\left\|x_{n}-T_{n} G v_{n}\right\|^{2} \\
& +2 s_{n}\left\|\gamma Q x_{n}-V T_{n} G v_{n}\right\|\left\|z_{n}-p\right\| \\
& \leq \beta_{n}\left\|x_{n}-p\right\|^{2}+\left(1-\beta_{n}\right)\left\|v_{n}-p\right\|^{2} \\
& -\beta_{n}\left(1-\beta_{n}\right)\left\|x_{n}-T_{n} G v_{n}\right\|^{2} \\
& +2 s_{n}\left\|\gamma Q x_{n}-V T_{n} G v_{n}\right\|\left\|z_{n}-p\right\| \\
& \leq \beta_{n}\left\|x_{n}-p\right\|^{2}+\left(1-\beta_{n}\right)\left\|x_{n}-p\right\|^{2} \\
& -\beta_{n}\left(1-\beta_{n}\right)\left\|x_{n}-T_{n} G v_{n}\right\|^{2} \\
& +2 s_{n}\left\|\gamma Q x_{n}-V T_{n} G v_{n}\right\|\left\|z_{n}-p\right\| \\
& =\left\|x_{n}-p\right\|^{2}-\beta_{n}\left(1-\beta_{n}\right)\left\|x_{n}-T_{n} G v_{n}\right\|^{2} \\
& +2 s_{n}\left\|\gamma Q x_{n}-V T_{n} G v_{n}\right\|\left\|z_{n}-p\right\| \text {, }
\end{aligned}
$$

and hence

$$
\begin{aligned}
& \left\|y_{n}-p\right\|^{2} \\
& \leq\left(1-\alpha_{n}\right)\left\|x_{n}-p\right\|^{2}+\alpha_{n}\left\|k_{n}-p\right\|^{2} \\
& \leq\left(1-\alpha_{n}\right)\left\|x_{n}-p\right\|^{2}+\alpha_{n}\left[\left(1+\gamma_{n}\right)\left\|z_{n}-p\right\|^{2}+c_{n}\right] \\
& \leq\left(1-\alpha_{n}\right)\left\|x_{n}-p\right\|^{2} \\
& \quad+\alpha_{n}\left[( 1 + \gamma _ { n } ) \left(\left\|x_{n}-p\right\|^{2}\right.\right. \\
& \quad-\beta_{n}\left(1-\beta_{n}\right)\left\|x_{n}-T_{n} G v_{n}\right\|^{2} \\
& \left.\left.\quad+2 s_{n}\left\|\gamma Q x_{n}-V T_{n} G v_{n}\right\|\left\|z_{n}-p\right\|\right)+c_{n}\right]
\end{aligned}
$$




$$
\begin{aligned}
\leq & \left(1-\alpha_{n}\right)\left\|x_{n}-p\right\|^{2}+\alpha_{n}\left(1+\gamma_{n}\right) \\
& \times\left(\left\|x_{n}-p\right\|^{2}-\beta_{n}\left(1-\beta_{n}\right)\left\|x_{n}-T_{n} G v_{n}\right\|^{2}\right. \\
& \left.\quad+2 s_{n}\left\|\gamma \mathrm{Q} x_{n}-V T_{n} G v_{n}\right\|\left\|z_{n}-p\right\|\right)+c_{n} \\
\leq & \left(1-\alpha_{n}\right)\left\|x_{n}-p\right\|^{2}+\alpha_{n}\left(1+\gamma_{n}\right)\left\|x_{n}-p\right\|^{2} \\
& -\alpha_{n}\left(1+\gamma_{n}\right) \beta_{n}\left(1-\beta_{n}\right)\left\|x_{n}-T_{n} G v_{n}\right\|^{2} \\
& +\left(1+\gamma_{n}\right) 2 s_{n}\left\|\gamma Q x_{n}-V T_{n} G v_{n}\right\|\left\|z_{n}-p\right\|+c_{n} \\
\leq & \left(1+\gamma_{n}\right)\left\|x_{n}-p\right\|^{2}-\alpha_{n}\left(1+\gamma_{n}\right) \\
& \times \beta_{n}\left(1-\beta_{n}\right)\left\|x_{n}-T_{n} G v_{n}\right\|^{2} \\
& +2 s_{n}\left(1+\gamma_{n}\right)\left\|\gamma Q x_{n}-V T_{n} G v_{n}\right\|\left\|z_{n}-p\right\|+c_{n} .
\end{aligned}
$$

So, it follows that

$$
\begin{aligned}
\alpha(1 & \left.+\gamma_{n}\right) a(1-\widehat{a})\left\|x_{n}-T_{n} G v_{n}\right\|^{2} \\
\leq & \alpha_{n}\left(1+\gamma_{n}\right) \beta_{n}\left(1-\beta_{n}\right)\left\|x_{n}-T_{n} G v_{n}\right\|^{2} \\
\leq & \left\|x_{n}-p\right\|^{2}-\left\|y_{n}-p\right\|^{2}+\gamma_{n}\left\|x_{n}-p\right\|^{2} \\
& +2 s_{n}\left(1+\gamma_{n}\right)\left\|\gamma Q x_{n}-V T_{n} G v_{n}\right\|\left\|z_{n}-p\right\|+c_{n} \\
\leq & \left\|x_{n}-y_{n}\right\|\left(\left\|x_{n}-p\right\|+\left\|y_{n}-p\right\|\right) \\
& +\gamma_{n}\left\|x_{n}-p\right\|^{2}+2 s_{n}\left(1+\gamma_{n}\right) \\
& \times\left\|\gamma Q x_{n}-V T_{n} G v_{n}\right\|\left\|z_{n}-p\right\|+c_{n} .
\end{aligned}
$$

Since $\lim _{n \rightarrow \infty} s_{n}=0, \lim _{n \rightarrow \infty} \gamma_{n}=0$ and $\lim _{n \rightarrow \infty} c_{n}=0$, it follows from (70) and the boundedness of $\left\{x_{n}\right\},\left\{y_{n}\right\},\left\{z_{n}\right\}$, and $\left\{v_{n}\right\}$ that

$$
\lim _{n \rightarrow \infty}\left\|x_{n}-T_{n} G v_{n}\right\|=0
$$

Note that

$$
\begin{aligned}
& \left\|z_{n}-x_{n}\right\| \\
& \quad=\left\|\left(1-\beta_{n}\right)\left(T_{n} G v_{n}-x_{n}\right)+s_{n}\left(\gamma Q x_{n}-V T_{n} G v_{n}\right)\right\| \\
& \quad \leq\left(1-\beta_{n}\right)\left\|T_{n} G v_{n}-x_{n}\right\|+s_{n}\left\|\gamma Q x_{n}-V T_{n} G v_{n}\right\| \\
& \quad \leq\left\|T_{n} G v_{n}-x_{n}\right\|+s_{n}\left\|\gamma Q x_{n}-V T_{n} G v_{n}\right\| .
\end{aligned}
$$

Hence, it follows from (76) and $\lim _{n \rightarrow \infty} s_{n}=0$ that

$$
\lim _{n \rightarrow \infty}\left\|x_{n}-z_{n}\right\|=0 \text {. }
$$

Note that

$$
\left\|k_{n}-z_{n}\right\| \leq\left\|k_{n}-x_{n}\right\|+\left\|x_{n}-z_{n}\right\| .
$$

Thus, we deduce from (72) and (78) that

$$
\lim _{n \rightarrow \infty}\left\|k_{n}-z_{n}\right\|=0 \text {. }
$$

Since $k_{n}-z_{n}=\left(1-\delta_{n}\right)\left(S^{n} z_{n}-z_{n}\right)$ and $k \leq \delta_{n} \leq d<1$, we have

$$
(1-d)\left\|S^{n} z_{n}-z_{n}\right\| \leq\left(1-\delta_{n}\right)\left\|S^{n} z_{n}-z_{n}\right\|=\left\|k_{n}-z_{n}\right\|,
$$

which together with (80), yields

$$
\lim _{n \rightarrow \infty}\left\|S^{n} z_{n}-z_{n}\right\|=0
$$

Step 3. We prove that $\left\|x_{n}-u_{n}\right\| \rightarrow 0,\left\|x_{n}-v_{n}\right\| \rightarrow 0, \| v_{n}-$ $G v_{n}\|\rightarrow 0,\| v_{n}-P_{C}(I-(2 / L) \nabla f) v_{n} \| \rightarrow 0$ and $\left\|z_{n}-S z_{n}\right\| \rightarrow 0$ as $n \rightarrow \infty$.

Indeed, from (58), (60), and $\gamma l<\bar{\gamma}$, it follows that

$$
\begin{aligned}
& \left\|z_{n}-p\right\|^{2} \\
& =\| \beta_{n}\left(x_{n}-p\right)+s_{n} \gamma\left(Q x_{n}-Q p\right) \\
& +\left[\left(1-\beta_{n}\right) I-s_{n} V\right]\left(T_{n} G v_{n}-p\right)+s_{n}(\gamma Q-V) p \|^{2} \\
& \leq \| \beta_{n}\left(x_{n}-p\right)+s_{n} \gamma\left(Q x_{n}-Q p\right) \\
& +\left[\left(1-\beta_{n}\right) I-s_{n} V\right]\left(T_{n} G v_{n}-p\right) \|^{2} \\
& +2 s_{n}\left\langle(\gamma Q-V) p, z_{n}-p\right\rangle \\
& \leq\left[\beta_{n}\left\|x_{n}-p\right\|+s_{n} \gamma\left\|Q x_{n}-Q p\right\|\right. \\
& \left.+\left(1-\beta_{n}-s_{n} \bar{\gamma}\right)\left\|T_{n} G v_{n}-p\right\|\right]^{2} \\
& +2 s_{n}\left\langle(\gamma Q-V) p, z_{n}-p\right\rangle \\
& \leq\left[\beta_{n}\left\|x_{n}-p\right\|+s_{n} \gamma l\left\|x_{n}-p\right\|+\left(1-\beta_{n}-s_{n} \bar{\gamma}\right)\left\|G v_{n}-p\right\|\right]^{2} \\
& +2 s_{n}\left\langle(\gamma Q-V) p, z_{n}-p\right\rangle \\
& =\left[\left(\beta_{n}+s_{n} \gamma l\right)\left\|x_{n}-p\right\|+\left(1-\beta_{n}-s_{n} \bar{\gamma}\right)\left\|G v_{n}-p\right\|\right]^{2} \\
& +2 s_{n}\left\langle(\gamma Q-V) p, z_{n}-p\right\rangle \\
& \leq\left[\left(\beta_{n}+s_{n} \bar{\gamma}\right)\left\|x_{n}-p\right\|+\left(1-\beta_{n}-s_{n} \bar{\gamma}\right)\left\|G v_{n}-p\right\|\right]^{2} \\
& +2 s_{n}\left\langle(\gamma Q-V) p, z_{n}-p\right\rangle \\
& \leq\left(\beta_{n}+s_{n} \bar{\gamma}\right)\left\|x_{n}-p\right\|^{2}+\left(1-\beta_{n}-s_{n} \bar{\gamma}\right)\left\|G v_{n}-p\right\|^{2} \\
& +2 s_{n}\|(\gamma Q-V) p\|\left\|z_{n}-p\right\| \\
& \leq\left(\beta_{n}+s_{n} \bar{\gamma}\right)\left\|x_{n}-p\right\|^{2}+\left(1-\beta_{n}-s_{n} \bar{\gamma}\right)\left\|v_{n}-p\right\|^{2} \\
& +2 s_{n}\|(\gamma Q-V) p\|\left\|z_{n}-p\right\| \\
& \leq\left(\beta_{n}+s_{n} \bar{\gamma}\right)\left\|x_{n}-p\right\|^{2}+\left(1-\beta_{n}-s_{n} \bar{\gamma}\right)\left\|u_{n}-p\right\|^{2} \\
& +2 s_{n}\|(\gamma Q-V) p\|\left\|z_{n}-p\right\| .
\end{aligned}
$$


Next let us show that

$$
\lim _{n \rightarrow \infty}\left\|x_{n}-u_{n}\right\|=0
$$

For $p \in \Omega$, we find that

$$
\begin{aligned}
\left\|u_{n}-p\right\|^{2} & =\left\|S_{r_{n}}^{(\Theta, \varphi)}\left(I-r_{n} A\right) x_{n}-S_{r_{n}}^{(\Theta, \varphi)}\left(I-r_{n} A\right) p\right\|^{2} \\
& \leq\left\|\left(I-r_{n} A\right) x_{n}-\left(I-r_{n} A\right) p\right\|^{2} \\
& =\left\|x_{n}-p-r_{n}\left(A x_{n}-A p\right)\right\|^{2} \\
& \leq\left\|x_{n}-p\right\|^{2}+r_{n}\left(r_{n}-2 \zeta\right)\left\|A x_{n}-A p\right\|^{2} .
\end{aligned}
$$

Combining (83) and (85), we obtain

$$
\begin{aligned}
& \left\|z_{n}-p\right\|^{2} \\
& \leq\left(\beta_{n}+s_{n} \bar{\gamma}\right)\left\|x_{n}-p\right\|^{2}+\left(1-\beta_{n}-s_{n} \bar{\gamma}\right)\left\|u_{n}-p\right\|^{2} \\
& \quad+2 s_{n}\|(\gamma Q-V) p\|\left\|z_{n}-p\right\| \\
& \leq\left(\beta_{n}+s_{n} \bar{\gamma}\right)\left\|x_{n}-p\right\|^{2}+\left(1-\beta_{n}-s_{n} \bar{\gamma}\right) \\
& \quad \times\left[\left\|x_{n}-p\right\|^{2}+r_{n}\left(r_{n}-2 \zeta\right)\left\|A x_{n}-A p\right\|^{2}\right] \\
& \quad+2 s_{n}\|(\gamma Q-V) p\|\left\|z_{n}-p\right\| \\
& =\left\|x_{n}-p\right\|^{2}+\left(1-\beta_{n}-s_{n} \bar{\gamma}\right) r_{n}\left(r_{n}-2 \zeta\right)\left\|A x_{n}-A p\right\|^{2} \\
& \quad+2 s_{n}\|(\gamma Q-V) p\|\left\|z_{n}-p\right\|,
\end{aligned}
$$

which immediately implies that

$$
\begin{aligned}
& \left(1-\widehat{a}-s_{n} \bar{\gamma}\right) c(2 \zeta-\widehat{c})\left\|A x_{n}-A p\right\|^{2} \\
& \leq\left(1-\beta_{n}-s_{n} \bar{\gamma}\right) r_{n}\left(2 \zeta-r_{n}\right)\left\|A x_{n}-A p\right\|^{2} \\
& \leq\left\|x_{n}-p\right\|^{2}-\left\|z_{n}-p\right\|^{2}+2 s_{n}\|(\gamma Q-V) p\|\left\|z_{n}-p\right\| \\
& \leq\left\|x_{n}-z_{n}\right\|\left(\left\|x_{n}-p\right\|+\left\|z_{n}-p\right\|\right) \\
& \quad+2 s_{n}\|(\gamma Q-V) p\|\left\|z_{n}-p\right\| .
\end{aligned}
$$

Since $\lim _{n \rightarrow \infty} s_{n}=0$ and $\left\{x_{n}\right\}$ and $\left\{z_{n}\right\}$ are bounded sequences, it follows from (78) that

$$
\lim _{n \rightarrow \infty}\left\|A x_{n}-A p\right\|=0
$$

Furthermore, from the firm nonexpansivity of $S_{r_{n}}^{(\Theta, \varphi)}$, we have

$$
\begin{aligned}
& \left\|u_{n}-p\right\|^{2} \\
& =\left\|S_{r_{n}}^{(\Theta, \varphi)}\left(I-r_{n} A\right) x_{n}-S_{r_{n}}^{(\Theta, \varphi)}\left(I-r_{n} A\right) p\right\|^{2} \\
& \leq\left\langle\left(I-r_{n} A\right) x_{n}-\left(I-r_{n} A\right) p, u_{n}-p\right\rangle \\
& =\frac{1}{2}\left[\left\|\left(I-r_{n} A\right) x_{n}-\left(I-r_{n} A\right) p\right\|^{2}+\left\|u_{n}-p\right\|^{2}\right. \\
& \left.\quad-\left\|\left(I-r_{n} A\right) x_{n}-\left(I-r_{n} A\right) p-\left(u_{n}-p\right)\right\|^{2}\right] \\
& \leq \frac{1}{2}\left[\left\|x_{n}-p\right\|^{2}+\left\|u_{n}-p\right\|^{2}\right. \\
& \left.\quad-\left\|x_{n}-u_{n}-r_{n}\left(A x_{n}-A p\right)\right\|^{2}\right] \\
& =\frac{1}{2}\left[\left\|x_{n}-p\right\|^{2}+\left\|u_{n}-p\right\|^{2}-\left\|x_{n}-u_{n}\right\|^{2}\right. \\
& \left.\quad+2 r_{n}\left\langle A x_{n}-A p, x_{n}-u_{n}\right\rangle-r_{n}^{2}\left\|A x_{n}-A p\right\|^{2}\right],
\end{aligned}
$$

which leads to

$$
\begin{aligned}
\left\|u_{n}-p\right\|^{2} \leq & \left\|x_{n}-p\right\|^{2}-\left\|x_{n}-u_{n}\right\|^{2} \\
& +2 r_{n}\left\|A x_{n}-A p\right\|\left\|x_{n}-u_{n}\right\| .
\end{aligned}
$$

From (83) and (90), we have

$$
\left\|z_{n}-p\right\|^{2}
$$

$$
\begin{aligned}
\leq & \left(\beta_{n}+s_{n} \bar{\gamma}\right)\left\|x_{n}-p\right\|^{2}+\left(1-\beta_{n}-s_{n} \bar{\gamma}\right)\left\|u_{n}-p\right\|^{2} \\
& +2 s_{n}\|(\gamma Q-V) p\|\left\|z_{n}-p\right\| \\
\leq & \left(\beta_{n}+s_{n} \bar{\gamma}\right)\left\|x_{n}-p\right\|^{2}+\left(1-\beta_{n}-s_{n} \bar{\gamma}\right) \\
& \times\left[\left\|x_{n}-p\right\|^{2}-\left\|x_{n}-u_{n}\right\|^{2}\right. \\
& \left.+2 r_{n}\left\|A x_{n}-A p\right\|\left\|x_{n}-u_{n}\right\|\right] \\
& +2 s_{n}\|(\gamma Q-V) p\|\left\|z_{n}-p\right\| \\
\leq & \left\|x_{n}-p\right\|^{2}-\left(1-\beta_{n}-s_{n} \bar{\gamma}\right)\left\|x_{n}-u_{n}\right\|^{2} \\
& +2 r_{n}\left\|A x_{n}-A p\right\|\left\|x_{n}-u_{n}\right\| \\
& +2 s_{n}\|(\gamma Q-V) p\|\left\|z_{n}-p\right\|,
\end{aligned}
$$


which hence implies that

$$
\begin{aligned}
& \left(1-\widehat{a}-s_{n} \bar{\gamma}\right)\left\|x_{n}-u_{n}\right\|^{2} \\
& \leq\left(1-\beta_{n}-s_{n} \bar{\gamma}\right)\left\|x_{n}-u_{n}\right\|^{2} \\
& \leq\left\|x_{n}-p\right\|^{2}-\left\|z_{n}-p\right\|^{2}+2 r_{n}\left\|A x_{n}-A p\right\|\left\|x_{n}-u_{n}\right\| \\
& \quad+2 s_{n}\|(\gamma Q-V) p\|\left\|z_{n}-p\right\| \\
& \leq\left\|x_{n}-z_{n}\right\|\left(\left\|x_{n}-p\right\|+\left\|z_{n}-p\right\|\right) \\
& \quad+2 r_{n}\left\|A x_{n}-A p\right\|\left\|x_{n}-u_{n}\right\| \\
& \quad+2 s_{n}\|(\gamma Q-V) p\|\left\|z_{n}-p\right\| .
\end{aligned}
$$

Since $\lim _{n \rightarrow \infty} s_{n}=0$ and $\left\{x_{n}\right\},\left\{u_{n}\right\}$, and $\left\{z_{n}\right\}$ are bounded sequences, it follows from (78) and (88) that (84) holds.

Next we show that $\lim _{n \rightarrow \infty}\left\|B_{i} \Lambda_{n}^{i} u_{n}-B_{i} p\right\|=0, i=$ $1,2, \ldots, N$. As a matter of fact, observe that

$$
\begin{aligned}
& \left\|\Lambda_{n}^{i} u_{n}-p\right\|^{2} \\
& =\left\|J_{R_{i}, \lambda_{i, n}}\left(I-\lambda_{i, n} B_{i}\right) \Lambda_{n}^{i-1} u_{n}-J_{R_{i}, \lambda_{i, n}}\left(I-\lambda_{i, n} B_{i}\right) p\right\|^{2} \\
& \leq\left\|\left(I-\lambda_{i, n} B_{i}\right) \Lambda_{n}^{i-1} u_{n}-\left(I-\lambda_{i, n} B_{i}\right) p\right\|^{2} \\
& \leq\left\|\Lambda_{n}^{i-1} u_{n}-p\right\|^{2}+\lambda_{i, n}\left(\lambda_{i, n}-2 \eta_{i}\right)\left\|B_{i} \Lambda_{n}^{i-1} u_{n}-B_{i} p\right\|^{2} \\
& \leq\left\|u_{n}-p\right\|^{2}+\lambda_{i, n}\left(\lambda_{i, n}-2 \eta_{i}\right)\left\|B_{i} \Lambda_{n}^{i-1} u_{n}-B_{i} p\right\|^{2} \\
& \leq\left\|x_{n}-p\right\|^{2}+\lambda_{i, n}\left(\lambda_{i, n}-2 \eta_{i}\right)\left\|B_{i} \Lambda_{n}^{i-1} u_{n}-B_{i} p\right\|^{2} .
\end{aligned}
$$

Combining (60), (83), and (93), we have

$$
\begin{aligned}
&\left\|z_{n}-p\right\|^{2} \\
& \leq\left(\beta_{n}+s_{n} \bar{\gamma}\right)\left\|x_{n}-p\right\|^{2}+\left(1-\beta_{n}-s_{n} \bar{\gamma}\right)\left\|v_{n}-p\right\|^{2} \\
&+2 s_{n}\|(\gamma Q-V) p\|\left\|z_{n}-p\right\| \\
& \leq\left(\beta_{n}+s_{n} \bar{\gamma}\right)\left\|x_{n}-p\right\|^{2}+\left(1-\beta_{n}-s_{n} \bar{\gamma}\right)\left\|\Lambda_{n}^{i} u_{n}-p\right\|^{2} \\
&+2 s_{n}\|(\gamma Q-V) p\|\left\|z_{n}-p\right\| \\
& \leq\left(\beta_{n}+s_{n} \bar{\gamma}\right)\left\|x_{n}-p\right\|^{2}+\left(1-\beta_{n}-s_{n} \bar{\gamma}\right) \\
& \times\left[\left\|x_{n}-p\right\|^{2}+\lambda_{i, n}\left(\lambda_{i, n}-2 \eta_{i}\right)\left\|B_{i} \Lambda_{n}^{i-1} u_{n}-B_{i} p\right\|^{2}\right] \\
&+2 s_{n}\|(\gamma Q-V) p\|\left\|z_{n}-p\right\| \\
&=\left\|x_{n}-p\right\|^{2}+\left(1-\beta_{n}-s_{n} \bar{\gamma}\right) \lambda_{i, n}\left(\lambda_{i, n}-2 \eta_{i}\right) \\
& \times\left\|B_{i} \Lambda_{n}^{i-1} u_{n}-B_{i} p\right\|^{2}+2 s_{n}\|(\gamma Q-V) p\|\left\|z_{n}-p\right\|,
\end{aligned}
$$

which together with $\left\{\lambda_{i, n}\right\} \subset\left[a_{i}, b_{i}\right] \subset\left(0,2 \eta_{i}\right)$, for all $i \in$ $\{1,2, \ldots, N\}$, implies that

$$
\begin{aligned}
& \left(1-\widehat{a}-s_{n} \bar{\gamma}\right) a_{i}\left(2 \eta_{i}-b_{i}\right)\left\|B_{i} \Lambda_{n}^{i-1} u_{n}-B_{i} p\right\|^{2} \\
& \leq\left(1-\beta_{n}-s_{n} \bar{\gamma}\right) \lambda_{i, n}\left(\lambda_{i, n}-2 \eta_{i}\right)\left\|B_{i} \Lambda_{n}^{i-1} u_{n}-B_{i} p\right\|^{2} \\
& \leq\left\|x_{n}-p\right\|^{2}-\left\|z_{n}-p\right\|^{2}+2 s_{n}\|(\gamma Q-V) p\|\left\|z_{n}-p\right\| \\
& \leq\left\|x_{n}-z_{n}\right\|\left(\left\|x_{n}-p\right\|+\left\|z_{n}-p\right\|\right) \\
& \quad+2 s_{n}\|(\gamma Q-V) p\|\left\|z_{n}-p\right\| .
\end{aligned}
$$

Since $\lim _{n \rightarrow \infty} s_{n}=0$ and $\left\{x_{n}\right\}$ and $\left\{z_{n}\right\}$ are bounded sequences, it follows from (78) that

$$
\lim _{n \rightarrow \infty}\left\|B_{i} \Lambda_{n}^{i-1} u_{n}-B_{i} p\right\|=0, \quad i=1,2, \ldots, N
$$

By Lemma 16(a) and Lemma 17, we obtain

$$
\begin{aligned}
& \left\|\Lambda_{n}^{i} u_{n}-p\right\|^{2} \\
& =\left\|J_{R_{i}, \lambda_{i, n}}\left(I-\lambda_{i, n} B_{i}\right) \Lambda_{n}^{i-1} u_{n}-J_{R_{i}, \lambda_{i, n}}\left(I-\lambda_{i, n} B_{i}\right) p\right\|^{2} \\
& \leq\left\langle\left(I-\lambda_{i, n} B_{i}\right) \Lambda_{n}^{i-1} u_{n}-\left(I-\lambda_{i, n} B_{i}\right) p, \Lambda_{n}^{i} u_{n}-p\right\rangle \\
& =\frac{1}{2}\left(\left\|\left(I-\lambda_{i, n} B_{i}\right) \Lambda_{n}^{i-1} u_{n}-\left(I-\lambda_{i, n} B_{i}\right) p\right\|^{2}+\left\|\Lambda_{n}^{i} u_{n}-p\right\|^{2}\right. \\
& \quad-\|\left(I-\lambda_{i, n} B_{i}\right) \Lambda_{n}^{i-1} u_{n} \\
& \left.\quad-\left(I-\lambda_{i, n} B_{i}\right) p-\left(\Lambda_{n}^{i} u_{n}-p\right) \|^{2}\right) \\
& \leq \frac{1}{2}\left(\left\|\Lambda_{n}^{i-1} u_{n}-p\right\|^{2}+\left\|\Lambda_{n}^{i} u_{n}-p\right\|^{2}\right. \\
& \left.\quad-\left\|\Lambda_{n}^{i-1} u_{n}-\Lambda_{n}^{i} u_{n}-\lambda_{i, n}\left(B_{i} \Lambda_{n}^{i-1} u_{n}-B_{i} p\right)\right\|^{2}\right) \\
& \leq \frac{1}{2}\left(\left\|u_{n}-p\right\|^{2}+\left\|\Lambda_{n}^{i} u_{n}-p\right\|^{2}\right. \\
& \left.\quad-\left\|\Lambda_{n}^{i-1} u_{n}-\Lambda_{n}^{i} u_{n}-\lambda_{i, n}\left(B_{i} \Lambda_{n}^{i-1} u_{n}-B_{i} p\right)\right\|^{2}\right) \\
& \leq \frac{1}{2}\left(\left\|x_{n}-p\right\|^{2}+\left\|\Lambda_{n}^{i} u_{n}-p\right\|^{2}\right. \\
& \left.\quad-\left\|\Lambda_{n}^{i-1} u_{n}-\Lambda_{n}^{i} u_{n}-\lambda_{i, n}\left(B_{i} \Lambda_{n}^{i-1} u_{n}-B_{i} p\right)\right\|^{2}\right),
\end{aligned}
$$


which implies

$$
\begin{aligned}
& \left\|\Lambda_{n}^{i} u_{n}-p\right\|^{2} \\
& \leq\left\|x_{n}-p\right\|^{2}-\left\|\Lambda_{n}^{i-1} u_{n}-\Lambda_{n}^{i} u_{n}-\lambda_{i, n}\left(B_{i} \Lambda_{n}^{i-1} u_{n}-B_{i} p\right)\right\|^{2} \\
& =\left\|x_{n}-p\right\|^{2}-\left\|\Lambda_{n}^{i-1} u_{n}-\Lambda_{n}^{i} u_{n}\right\|^{2}-\lambda_{i, n}^{2}\left\|B_{i} \Lambda_{n}^{i-1} u_{n}-B_{i} p\right\|^{2} \\
& \quad+2 \lambda_{i, n}\left\langle\Lambda_{n}^{i-1} u_{n}-\Lambda_{n}^{i} u_{n}, B_{i} \Lambda_{n}^{i-1} u_{n}-B_{i} p\right\rangle \\
& \leq\left\|x_{n}-p\right\|^{2}-\left\|\Lambda_{n}^{i-1} u_{n}-\Lambda_{n}^{i} u_{n}\right\|^{2} \\
& \quad+2 \lambda_{i, n}\left\|\Lambda_{n}^{i-1} u_{n}-\Lambda_{n}^{i} u_{n}\right\|\left\|B_{i} \Lambda_{n}^{i-1} u_{n}-B_{i} p\right\| .
\end{aligned}
$$

Combining (60), (83), and (98), we have

$$
\begin{aligned}
\| z_{n}- & p \|^{2} \\
\leq & \left(\beta_{n}+s_{n} \bar{\gamma}\right)\left\|x_{n}-p\right\|^{2}+\left(1-\beta_{n}-s_{n} \bar{\gamma}\right)\left\|v_{n}-p\right\|^{2} \\
& +2 s_{n}\|(\gamma Q-V) p\|\left\|z_{n}-p\right\| \\
\leq & \left(\beta_{n}+s_{n} \bar{\gamma}\right)\left\|x_{n}-p\right\|^{2}+\left(1-\beta_{n}-s_{n} \bar{\gamma}\right)\left\|\Lambda_{n}^{i} u_{n}-p\right\|^{2} \\
& +2 s_{n}\|(\gamma Q-V) p\|\left\|z_{n}-p\right\| \\
\leq & \left(\beta_{n}+s_{n} \bar{\gamma}\right)\left\|x_{n}-p\right\|^{2}+\left(1-\beta_{n}-s_{n} \bar{\gamma}\right) \\
& \times\left[\left\|x_{n}-p\right\|^{2}-\left\|\Lambda_{n}^{i-1} u_{n}-\Lambda_{n}^{i} u_{n}\right\|^{2}\right. \\
& \left.+2 \lambda_{i, n}\left\|\Lambda_{n}^{i-1} u_{n}-\Lambda_{n}^{i} u_{n}\right\|\left\|B_{i} \Lambda_{n}^{i-1} u_{n}-B_{i} p\right\|\right] \\
& +2 s_{n}\|(\gamma Q-V) p\|\left\|z_{n}-p\right\| \\
\leq & \left\|x_{n}-p\right\|^{2}-\left(1-\beta_{n}-s_{n} \bar{\gamma}\right)\left\|\Lambda_{n}^{i-1} u_{n}-\Lambda_{n}^{i} u_{n}\right\|^{2} \\
& +2 \lambda_{i, n}\left\|\Lambda_{n}^{i-1} u_{n}-\Lambda_{n}^{i} u_{n}\right\|\left\|B_{i} \Lambda_{n}^{i-1} u_{n}-B_{i} p\right\| \\
& +2 s_{n}\|(\gamma Q-V) p\|\left\|z_{n}-p\right\| .
\end{aligned}
$$

So, we conclude that

$$
\begin{aligned}
& \left(1-\widehat{a}-s_{n} \bar{\gamma}\right)\left\|\Lambda_{n}^{i-1} u_{n}-\Lambda_{n}^{i} u_{n}\right\|^{2} \\
& \leq\left(1-\beta_{n}-s_{n} \bar{\gamma}\right)\left\|\Lambda_{n}^{i-1} u_{n}-\Lambda_{n}^{i} u_{n}\right\|^{2} \\
& \leq\left\|x_{n}-p\right\|^{2}-\left\|z_{n}-p\right\|^{2} \\
& \quad+2 \lambda_{i, n}\left\|\Lambda_{n}^{i-1} u_{n}-\Lambda_{n}^{i} u_{n}\right\|\left\|B_{i} \Lambda_{n}^{i-1} u_{n}-B_{i} p\right\| \\
& \quad+2 s_{n}\|(\gamma Q-V) p\|\left\|z_{n}-p\right\| \\
& \leq \\
& \quad\left\|x_{n}-z_{n}\right\|\left(\left\|x_{n}-p\right\|+\left\|z_{n}-p\right\|\right) \\
& \quad+2 b_{i}\left\|\Lambda_{n}^{i-1} u_{n}-\Lambda_{n}^{i} u_{n}\right\|\left\|B_{i} \Lambda_{n}^{i-1} u_{n}-B_{i} p\right\| \\
& \quad+2 s_{n}\|(\gamma Q-V) p\|\left\|z_{n}-p\right\| .
\end{aligned}
$$

Since $\lim _{n \rightarrow \infty} s_{n}=0$ and $\left\{x_{n}\right\},\left\{z_{n}\right\}$, and $\left\{u_{n}\right\}$ are bounded, from (78) and (96) we get

$$
\lim _{n \rightarrow \infty}\left\|\Lambda_{n}^{i-1} u_{n}-\Lambda_{n}^{i} u_{n}\right\|=0
$$

From (101) we get

$$
\begin{aligned}
\left\|u_{n}-v_{n}\right\|= & \left\|\Lambda_{n}^{0} u_{n}-\Lambda_{n}^{N} u_{n}\right\| \\
\leq & \left\|\Lambda_{n}^{0} u_{n}-\Lambda_{n}^{1} u_{n}\right\|+\left\|\Lambda_{n}^{1} u_{n}-\Lambda_{n}^{2} u_{n}\right\| \\
& +\cdots+\left\|\Lambda_{n}^{N-1} u_{n}-\Lambda_{n}^{N} u_{n}\right\| \\
& \longrightarrow 0 \text { as } n \longrightarrow \infty .
\end{aligned}
$$

Taking into account that $\left\|x_{n}-v_{n}\right\| \leq\left\|x_{n}-u_{n}\right\|+\left\|u_{n}-v_{n}\right\|$, we conclude from (84) and (102) that

$$
\lim _{n \rightarrow \infty}\left\|x_{n}-v_{n}\right\|=0
$$

On the other hand, for simplicity, we write $\tilde{p}=T_{\nu_{2}}^{\Theta_{2}}(I-$ $\left.v_{2} A_{2}\right) p, \widetilde{v}_{n}=T_{v_{2}}^{\Theta_{2}}\left(I-v_{2} A_{2}\right) v_{n}$ and $w_{n}=G v_{n}=T_{v_{1}}^{\Theta_{1}}(I-$ $\left.v_{1} A_{1}\right) \widetilde{v}_{n}$ for all $n \geq 1$. Then

$$
\begin{aligned}
p & =G p=T_{v_{1}}^{\Theta_{1}}\left(I-v_{1} A_{1}\right) \tilde{p} \\
& =T_{v_{1}}^{\Theta_{1}}\left(I-v_{1} A_{1}\right) T_{v_{2}}^{\Theta_{2}}\left(I-v_{2} A_{2}\right) p .
\end{aligned}
$$

We now show that $\lim _{n \rightarrow \infty}\left\|G v_{n}-v_{n}\right\|=0$; that is, $\lim _{n \rightarrow \infty}\left\|w_{n}-v_{n}\right\|=0$. As a matter of fact, for $p \in \Omega$, it follows from (59), (60), and (83) that

$$
\begin{aligned}
\| z_{n}- & p \|^{2} \\
\leq & \left(\beta_{n}+s_{n} \bar{\gamma}\right)\left\|x_{n}-p\right\|^{2}+\left(1-\beta_{n}-s_{n} \bar{\gamma}\right)\left\|G v_{n}-p\right\|^{2} \\
& +2 s_{n}\|(\gamma Q-V) p\|\left\|z_{n}-p\right\| \\
= & \left(\beta_{n}+s_{n} \bar{\gamma}\right)\left\|x_{n}-p\right\|^{2}+\left(1-\beta_{n}-s_{n} \bar{\gamma}\right)\left\|w_{n}-p\right\|^{2} \\
& +2 s_{n}\|(\gamma Q-V) p\|\left\|z_{n}-p\right\|
\end{aligned}
$$




$$
\begin{aligned}
& \leq\left(\beta_{n}+s_{n} \bar{\gamma}\right)\left\|x_{n}-p\right\|^{2}+\left(1-\beta_{n}-s_{n} \bar{\gamma}\right) \\
& \times\left[\left\|\widetilde{v}_{n}-\tilde{p}\right\|^{2}+v_{1}\left(\nu_{1}-2 \zeta_{1}\right)\left\|A_{1} \widetilde{v}_{n}-A_{1} \tilde{p}\right\|^{2}\right] \\
& +2 s_{n}\|(\gamma Q-V) p\|\left\|z_{n}-p\right\| \\
& \leq\left(\beta_{n}+s_{n} \bar{\gamma}\right)\left\|x_{n}-p\right\|^{2}+\left(1-\beta_{n}-s_{n} \bar{\gamma}\right) \\
& \times\left[\left\|v_{n}-p\right\|^{2}+v_{2}\left(v_{2}-2 \zeta_{2}\right)\left\|A_{2} v_{n}-A_{2} p\right\|^{2}\right. \\
& \left.+v_{1}\left(v_{1}-2 \zeta_{1}\right)\left\|A_{1} \widetilde{v}_{n}-A_{1} \tilde{p}\right\|^{2}\right] \\
& +2 s_{n}\|(\gamma Q-V) p\|\left\|z_{n}-p\right\| \\
& \leq\left(\beta_{n}+s_{n} \bar{\gamma}\right)\left\|x_{n}-p\right\|^{2}+\left(1-\beta_{n}-s_{n} \bar{\gamma}\right) \\
& \times\left[\left\|x_{n}-p\right\|^{2}+v_{2}\left(v_{2}-2 \zeta_{2}\right)\left\|A_{2} v_{n}-A_{2} p\right\|^{2}\right. \\
& \left.+v_{1}\left(v_{1}-2 \zeta_{1}\right)\left\|A_{1} \widetilde{v}_{n}-A_{1} \tilde{p}\right\|^{2}\right] \\
& +2 s_{n}\|(\gamma Q-V) p\|\left\|z_{n}-p\right\| \\
& =\left\|x_{n}-p\right\|^{2}+\left(1-\beta_{n}-s_{n} \bar{\gamma}\right) \\
& \times\left[v_{2}\left(v_{2}-2 \zeta_{2}\right)\left\|A_{2} v_{n}-A_{2} p\right\|^{2}\right. \\
& \left.+v_{1}\left(v_{1}-2 \zeta_{1}\right)\left\|A_{1} \widetilde{v}_{n}-A_{1} \tilde{p}\right\|^{2}\right] \\
& +2 s_{n}\|(\gamma Q-V) p\|\left\|z_{n}-p\right\|,
\end{aligned}
$$

which immediately yields

$$
\begin{gathered}
\left(1-\widehat{a}-s_{n} \bar{\gamma}\right)\left[v_{2}\left(2 \zeta_{2}-v_{2}\right)\left\|A_{2} v_{n}-A_{2} p\right\|^{2}\right. \\
\left.+v_{1}\left(2 \zeta_{1}-v_{1}\right)\left\|A_{1} \widetilde{v}_{n}-A_{1} \tilde{p}\right\|^{2}\right] \\
\leq\left(1-\beta_{n}-s_{n} \bar{\gamma}\right)\left[v_{2}\left(2 \zeta_{2}-v_{2}\right)\left\|A_{2} v_{n}-A_{2} p\right\|^{2}\right. \\
\left.+v_{1}\left(2 \zeta_{1}-v_{1}\right)\left\|A_{1} \widetilde{v}_{n}-A_{1} \tilde{p}\right\|^{2}\right] \\
\leq\left\|x_{n}-p\right\|^{2}-\left\|z_{n}-p\right\|^{2}+2 s_{n}\|(\gamma Q-V) p\|\left\|z_{n}-p\right\| \\
\leq\left\|x_{n}-z_{n}\right\|\left(\left\|x_{n}-p\right\|+\left\|z_{n}-p\right\|\right) \\
\quad+2 s_{n}\|(\gamma Q-V) p\|\left\|z_{n}-p\right\| .
\end{gathered}
$$

Since $\lim _{n \rightarrow \infty} s_{n}=0$ and $\left\{x_{n}\right\}$ and $\left\{z_{n}\right\}$ are bounded, from (78) we get

$$
\lim _{n \rightarrow \infty}\left\|A_{2} v_{n}-A_{2} p\right\|=0, \quad \lim _{n \rightarrow \infty}\left\|A_{1} \widetilde{v}_{n}-A_{1} \widetilde{p}\right\|=0 .
$$

Also, in terms of the firm nonexpansivity of $T_{v_{k}}^{\Theta_{k}}$ and the $\zeta_{k^{-}}$ inverse strong monotonicity of $A_{k}$ for $k=1,2$, we obtain from $v_{k} \in\left(0,2 \zeta_{k}\right), k=1,2$ and (60) that

$$
\begin{aligned}
& \left\|\widetilde{v}_{n}-\tilde{p}\right\|^{2} \\
& =\left\|T_{v_{2}}^{\Theta_{2}}\left(I-v_{2} A_{2}\right) v_{n}-T_{v_{2}}^{\Theta_{2}}\left(I-v_{2} A_{2}\right) p\right\|^{2} \\
& \leq\left\langle\left(I-v_{2} A_{2}\right) v_{n}-\left(I-v_{2} A_{2}\right) p, \widetilde{v}_{n}-\tilde{p}\right\rangle \\
& =\frac{1}{2}\left[\left\|\left(I-v_{2} A_{2}\right) v_{n}-\left(I-v_{2} A_{2}\right) p\right\|^{2}+\left\|\widetilde{v}_{n}-\tilde{p}\right\|^{2}\right. \\
& \left.\quad-\left\|\left(I-v_{2} A_{2}\right) v_{n}-\left(I-v_{2} A_{2}\right) p-\left(\widetilde{v}_{n}-\tilde{p}\right)\right\|^{2}\right] \\
& \leq \frac{1}{2}\left[\left\|v_{n}-p\right\|^{2}+\left\|\widetilde{v}_{n}-\tilde{p}\right\|^{2}\right. \\
& \left.\quad-\left\|\left(v_{n}-\widetilde{v}_{n}\right)-v_{2}\left(A_{2} v_{n}-A_{2} p\right)-(p-\widetilde{p})\right\|^{2}\right] \\
& =\frac{1}{2}\left[\left\|v_{n}-p\right\|^{2}+\left\|\widetilde{v}_{n}-\tilde{p}\right\|^{2}-\left\|\left(v_{n}-\widetilde{v}_{n}\right)-(p-\widetilde{p})\right\|^{2}\right. \\
& \quad+2 v_{2}\left\langle\left(v_{n}-\widetilde{v}_{n}\right)-(p-\tilde{p}), A_{2} v_{n}-A_{2} p\right\rangle \\
& \left.\quad-v_{2}^{2}\left\|A_{2} v_{n}-A_{2} p\right\|^{2}\right],
\end{aligned}
$$

$\left\|w_{n}-p\right\|^{2}$

$$
=\left\|T_{v_{1}}^{\Theta_{1}}\left(I-v_{1} A_{1}\right) \widetilde{v}_{n}-T_{v_{1}}^{\Theta_{1}}\left(I-v_{1} A_{1}\right) \tilde{p}\right\|^{2}
$$$$
\leq\left\langle\left(I-v_{1} A_{1}\right) \widetilde{v}_{n}-\left(I-v_{1} A_{1}\right) \tilde{p}, w_{n}-p\right\rangle
$$$$
=\frac{1}{2}\left[\left\|\left(I-v_{1} A_{1}\right) \widetilde{v}_{n}-\left(I-v_{1} A_{1}\right) \tilde{p}\right\|^{2}+\left\|w_{n}-p\right\|^{2}\right.
$$$$
\left.-\left\|\left(I-v_{1} A_{1}\right) \widetilde{v}_{n}-\left(I-v_{1} A_{1}\right) \tilde{p}-\left(w_{n}-p\right)\right\|^{2}\right]
$$$$
\leq \frac{1}{2}\left[\left\|\widetilde{v}_{n}-\tilde{p}\right\|^{2}+\left\|w_{n}-p\right\|^{2}-\left\|\left(\widetilde{v}_{n}-w_{n}\right)+(p-\tilde{p})\right\|^{2}\right.
$$$$
+2 v_{1}\left\langle A_{1} \widetilde{v}_{n}-A_{1} \widetilde{p},\left(\widetilde{v}_{n}-w_{n}\right)+(p-\widetilde{p})\right\rangle
$$$$
\left.-v_{1}^{2}\left\|A_{1} \widetilde{v}_{n}-A_{1} \tilde{p}\right\|^{2}\right]
$$

$\leq \frac{1}{2}\left[\left\|v_{n}-p\right\|^{2}+\left\|w_{n}-p\right\|^{2}-\left\|\left(\widetilde{v}_{n}-w_{n}\right)+(p-\widetilde{p})\right\|^{2}\right.$

$$
\left.+2 v_{1}\left\langle A_{1} \widetilde{v}_{n}-A_{1} \widetilde{p},\left(\widetilde{v}_{n}-w_{n}\right)+(p-\widetilde{p})\right\rangle\right] .
$$


Thus, we have

$$
\begin{aligned}
\left\|\widetilde{v}_{n}-\widetilde{p}\right\|^{2} \leq & \left\|v_{n}-p\right\|^{2}-\left\|\left(v_{n}-\widetilde{v}_{n}\right)-(p-\widetilde{p})\right\|^{2} \\
& +2 v_{2}\left\langle\left(v_{n}-\widetilde{v}_{n}\right)-(p-\widetilde{p}), A_{2} v_{n}-A_{2} p\right\rangle \\
& \quad-v_{2}^{2}\left\|A_{2} v_{n}-A_{2} p\right\|^{2}, \\
\left\|w_{n}-p\right\|^{2} \leq & \left\|v_{n}-p\right\|^{2}-\left\|\left(\widetilde{v}_{n}-w_{n}\right)+(p-\widetilde{p})\right\|^{2} \\
& +2 v_{1}\left\|A_{1} \widetilde{v}_{n}-A_{1} \widetilde{p}\right\|\left\|\left(\widetilde{v}_{n}-w_{n}\right)+(p-\widetilde{p})\right\| .
\end{aligned}
$$

Consequently, from (59), (105), and (109) it follows that

$$
\begin{aligned}
& \left\|z_{n}-p\right\|^{2} \\
& \leq\left(\beta_{n}+s_{n} \bar{\gamma}\right)\left\|x_{n}-p\right\|^{2}+\left(1-\beta_{n}-s_{n} \bar{\gamma}\right) \\
& \times\left[\left\|\widetilde{v}_{n}-\tilde{p}\right\|^{2}+v_{1}\left(v_{1}-2 \zeta_{1}\right)\left\|A_{1} \widetilde{v}_{n}-A_{1} \tilde{p}\right\|^{2}\right] \\
& +2 s_{n}\|(\gamma Q-V) p\|\left\|z_{n}-p\right\| \\
& \leq\left(\beta_{n}+s_{n} \bar{\gamma}\right)\left\|x_{n}-p\right\|^{2}+\left(1-\beta_{n}-s_{n} \bar{\gamma}\right)\left\|\widetilde{v}_{n}-\tilde{p}\right\|^{2} \\
& +2 s_{n}\|(\gamma Q-V) p\|\left\|z_{n}-p\right\| \\
& \leq\left(\beta_{n}+s_{n} \bar{\gamma}\right)\left\|x_{n}-p\right\|^{2}+\left(1-\beta_{n}-s_{n} \bar{\gamma}\right) \\
& \times\left[\left\|v_{n}-p\right\|^{2}-\left\|\left(v_{n}-\widetilde{v}_{n}\right)-(p-\widetilde{p})\right\|^{2}\right. \\
& +2 v_{2}\left\langle\left(v_{n}-\widetilde{v}_{n}\right)-(p-\widetilde{p}), A_{2} v_{n}-A_{2} p\right\rangle \\
& \left.-v_{2}^{2}\left\|A_{2} v_{n}-A_{2} p\right\|^{2}\right] \\
& +2 s_{n}\|(\gamma Q-V) p\|\left\|z_{n}-p\right\| \\
& \leq\left(\beta_{n}+s_{n} \bar{\gamma}\right)\left\|x_{n}-p\right\|^{2}+\left(1-\beta_{n}-s_{n} \bar{\gamma}\right) \\
& \times\left[\left\|x_{n}-p\right\|^{2}-\left\|\left(v_{n}-\widetilde{v}_{n}\right)-(p-\widetilde{p})\right\|^{2}\right. \\
& \left.+2 v_{2}\left\|\left(v_{n}-\widetilde{v}_{n}\right)-(p-\widetilde{p})\right\|\left\|A_{2} v_{n}-A_{2} p\right\|\right] \\
& +2 s_{n}\|(\gamma Q-V) p\|\left\|z_{n}-p\right\| \\
& \leq\left\|x_{n}-p\right\|^{2}-\left(1-\beta_{n}-s_{n} \bar{\gamma}\right)\left\|\left(v_{n}-\widetilde{v}_{n}\right)-(p-\widetilde{p})\right\|^{2} \\
& +2 v_{2}\left\|\left(v_{n}-\widetilde{v}_{n}\right)-(p-\tilde{p})\right\|\left\|A_{2} v_{n}-A_{2} p\right\| \\
& +2 s_{n}\|(\gamma Q-V) p\|\left\|z_{n}-p\right\|,
\end{aligned}
$$

which hence leads to

$$
\begin{aligned}
& \left(1-\widehat{a}-s_{n} \bar{\gamma}\right)\left\|\left(v_{n}-\widetilde{v}_{n}\right)-(p-\widetilde{p})\right\|^{2} \\
& \leq\left(1-\beta_{n}-s_{n} \bar{\gamma}\right)\left\|\left(v_{n}-\widetilde{v}_{n}\right)-(p-\widetilde{p})\right\|^{2} \\
& \leq\left\|x_{n}-p\right\|^{2}-\left\|z_{n}-p\right\|^{2} \\
& \quad+2 v_{2}\left\|\left(v_{n}-\widetilde{v}_{n}\right)-(p-\tilde{p})\right\|\left\|A_{2} v_{n}-A_{2} p\right\| \\
& \quad+2 s_{n}\|(\gamma Q-V) p\|\left\|z_{n}-p\right\| \\
& \leq\left\|x_{n}-z_{n}\right\|\left(\left\|x_{n}-p\right\|+\left\|z_{n}-p\right\|\right) \\
& \quad+2 v_{2}\left\|\left(v_{n}-\widetilde{v}_{n}\right)-(p-\widetilde{p})\right\|\left\|A_{2} v_{n}-A_{2} p\right\| \\
& \quad+2 s_{n}\|(\gamma Q-V) p\|\left\|z_{n}-p\right\| .
\end{aligned}
$$

Since $\lim _{n \rightarrow \infty} s_{n}=0$ and $\left\{x_{n}\right\},\left\{z_{n}\right\},\left\{v_{n}\right\}$, and $\left\{\widetilde{v}_{n}\right\}$ are bounded sequences, we conclude from (78) and (107) that

$$
\lim _{n \rightarrow \infty}\left\|\left(v_{n}-\widetilde{v}_{n}\right)-(p-\widetilde{p})\right\|=0
$$

Furthermore, from (59), (105), and (110) it follows that

$$
\begin{aligned}
& \left\|z_{n}-p\right\|^{2} \\
& \leq\left(\beta_{n}+s_{n} \bar{\gamma}\right)\left\|x_{n}-p\right\|^{2}+\left(1-\beta_{n}-s_{n} \bar{\gamma}\right) \\
& \quad \times\left\|w_{n}-p\right\|^{2}+2 s_{n}\|(\gamma Q-V) p\|\left\|z_{n}-p\right\| \\
& \leq\left(\beta_{n}+s_{n} \bar{\gamma}\right)\left\|x_{n}-p\right\|^{2}+\left(1-\beta_{n}-s_{n} \bar{\gamma}\right) \\
& \quad \times\left[\left\|v_{n}-p\right\|^{2}-\left\|\left(\widetilde{v}_{n}-w_{n}\right)+(p-\tilde{p})\right\|^{2}\right. \\
& \left.\quad+2 v_{1}\left\|A_{1} \widetilde{v}_{n}-A_{1} \tilde{p}\right\|\left\|\left(\widetilde{v}_{n}-w_{n}\right)+(p-\tilde{p})\right\|\right] \\
& \quad+2 s_{n}\|(\gamma Q-V) p\|\left\|z_{n}-p\right\| \\
& \leq\left(\beta_{n}+s_{n} \bar{\gamma}\right)\left\|x_{n}-p\right\|^{2}+\left(1-\beta_{n}-s_{n} \bar{\gamma}\right) \\
& \quad \times\left[\left\|x_{n}-p\right\|^{2}-\left\|\left(\widetilde{v}_{n}-w_{n}\right)+(p-\tilde{p})\right\|^{2}\right. \\
& \left.\quad+2 v_{1}\left\|A_{1} \widetilde{v}_{n}-A_{1} \tilde{p}\right\|\left\|\left(\widetilde{v}_{n}-w_{n}\right)+(p-\tilde{p})\right\|\right] \\
& \quad+2 s_{n}\|(\gamma Q-V) p\|\left\|z_{n}-p\right\| \\
& \leq\left\|x_{n}-p\right\|^{2}-\left(1-\beta_{n}-s_{n} \bar{\gamma}\right)\left\|\left(\widetilde{v}_{n}-w_{n}\right)+(p-\tilde{p})\right\|^{2} \\
& \quad+2 v_{1}\left\|A_{1} \widetilde{v}_{n}-A_{1} \tilde{p}\right\|\left\|\left(\widetilde{v}_{n}-w_{n}\right)+(p-\tilde{p})\right\| \\
& \quad+2 s_{n}\|(\gamma Q-V) p\|\left\|z_{n}-p\right\|,
\end{aligned}
$$


which hence yields

$$
\begin{aligned}
&\left(1-\widehat{a}-s_{n} \bar{\gamma}\right)\left\|\left(\widetilde{v}_{n}-w_{n}\right)+(p-\tilde{p})\right\|^{2} \\
& \leq\left(1-\beta_{n}-s_{n} \bar{\gamma}\right)\left\|\left(\widetilde{v}_{n}-w_{n}\right)+(p-\widetilde{p})\right\|^{2} \\
& \leq\left\|x_{n}-p\right\|^{2}-\left\|z_{n}-p\right\|^{2} \\
&+2 v_{1}\left\|A_{1} \widetilde{v}_{n}-A_{1} \tilde{p}\right\|\left\|\left(\widetilde{v}_{n}-w_{n}\right)+(p-\widetilde{p})\right\| \\
&+2 s_{n}\|(\gamma Q-V) p\|\left\|z_{n}-p\right\| \\
& \leq\left\|x_{n}-z_{n}\right\|\left(\left\|x_{n}-p\right\|+\left\|z_{n}-p\right\|\right) \\
&+2 v_{1}\left\|A_{1} \widetilde{v}_{n}-A_{1} \tilde{p}\right\|\left\|\left(\widetilde{v}_{n}-w_{n}\right)+(p-\widetilde{p})\right\| \\
&+2 s_{n}\|(\gamma Q-V) p\|\left\|z_{n}-p\right\| .
\end{aligned}
$$

Since $\lim _{n \rightarrow \infty} s_{n}=0$ and $\left\{x_{n}\right\},\left\{z_{n}\right\},\left\{w_{n}\right\}$, and $\left\{\widetilde{v}_{n}\right\}$ are bounded sequences, we conclude from (78) and (107) that

$$
\lim _{n \rightarrow \infty}\left\|\left(\widetilde{v}_{n}-w_{n}\right)+(p-\tilde{p})\right\|=0
$$

Note that

$$
\left\|v_{n}-w_{n}\right\| \leq\left\|\left(v_{n}-\widetilde{v}_{n}\right)-(p-\tilde{p})\right\|+\left\|\left(\widetilde{v}_{n}-w_{n}\right)+(p-\tilde{p})\right\| .
$$

Hence from (113) and (116) we get

$$
\lim _{n \rightarrow \infty}\left\|v_{n}-G v_{n}\right\|=\lim _{n \rightarrow \infty}\left\|v_{n}-w_{n}\right\|=0 .
$$

Observe that

$$
\begin{aligned}
\left\|v_{n}-T_{n} v_{n}\right\| & \leq\left\|v_{n}-x_{n}\right\|+\left\|x_{n}-T_{n} G v_{n}\right\|+\left\|T_{n} G v_{n}-T_{n} v_{n}\right\| \\
& \leq\left\|v_{n}-x_{n}\right\|+\left\|x_{n}-T_{n} G v_{n}\right\|+\left\|G v_{n}-v_{n}\right\| .
\end{aligned}
$$

Hence, from (76), (103), and (118) we have

$$
\lim _{n \rightarrow \infty}\left\|v_{n}-T_{n} v_{n}\right\|=0
$$

It is clear that

$$
\begin{aligned}
\left\|P_{C}\left(I-\lambda_{n} \nabla f\right) v_{n}-v_{n}\right\| & =\left\|s_{n} v_{n}+\left(1-s_{n}\right) T_{n} v_{n}-v_{n}\right\| \\
& =\left(1-s_{n}\right)\left\|T_{n} v_{n}-v_{n}\right\| \\
& \leq\left\|T_{n} v_{n}-v_{n}\right\|,
\end{aligned}
$$

where $s_{n}=\left(2-\lambda_{n} L\right) / 4 \in(0,1 / 2)$ for each $\lambda_{n} \in(0,2 / L)$. Hence we have

$$
\begin{aligned}
&\left\|P_{C}\left(I-\frac{2}{L} \nabla f\right) v_{n}-v_{n}\right\| \\
& \leq\left\|P_{C}\left(I-\frac{2}{L} \nabla f\right) v_{n}-P_{C}\left(I-\lambda_{n} \nabla f\right) v_{n}\right\| \\
& \quad+\left\|P_{C}\left(I-\lambda_{n} \nabla f\right) v_{n}-v_{n}\right\| \\
& \leq\left\|\left(I-\frac{2}{L} \nabla f\right) v_{n}-\left(I-\lambda_{n} \nabla f\right) v_{n}\right\| \\
& \quad+\left\|P_{C}\left(I-\lambda_{n} \nabla f\right) v_{n}-v_{n}\right\| \\
& \leq\left(\frac{2}{L}-\lambda_{n}\right)\left\|\nabla f\left(v_{n}\right)\right\|+\left\|T_{n} v_{n}-v_{n}\right\| .
\end{aligned}
$$

From the boundedness of $\left\{v_{n}\right\}, s_{n} \rightarrow 0\left(\Leftrightarrow \lambda_{n} \rightarrow 2 / L\right)$ and $\left\|T_{n} v_{n}-v_{n}\right\| \rightarrow 0$ (due to (120)), it follows that

$$
\lim _{n \rightarrow \infty}\left\|v_{n}-P_{C}\left(I-\frac{2}{L} \nabla f\right) v_{n}\right\|=0 .
$$

In addition, from (68) and (78), we have

$$
\begin{aligned}
\left\|z_{n+1}-z_{n}\right\| & \leq\left\|z_{n+1}-x_{n+1}\right\|+\left\|x_{n+1}-x_{n}\right\|+\left\|x_{n}-z_{n}\right\| \\
& \longrightarrow 0 \text { as } n \longrightarrow \infty .
\end{aligned}
$$

We note that

$$
\begin{aligned}
& \left\|S^{n} z_{n}-S^{n+1} z_{n}\right\| \\
& \leq\left\|S^{n} z_{n}-z_{n}\right\|+\left\|z_{n}-z_{n+1}\right\|+\left\|z_{n+1}-S^{n+1} z_{n+1}\right\| \\
& \quad+\left\|S^{n+1} z_{n+1}-S^{n+1} z_{n}\right\| .
\end{aligned}
$$

From (82), (124), and Lemma 22, we obtain

$$
\lim _{n \rightarrow \infty}\left\|S^{n} z_{n}-S^{n+1} z_{n}\right\|=0 .
$$

In the meantime, we note that

$$
\begin{aligned}
\left\|z_{n}-S z_{n}\right\| \leq & \left\|z_{n}-S^{n} z_{n}\right\|+\left\|S^{n} z_{n}-S^{n+1} z_{n}\right\| \\
& +\left\|S^{n+1} z_{n}-S z_{n}\right\| .
\end{aligned}
$$

From (82), (126), and the uniform continuity of $S$, we have

$$
\lim _{n \rightarrow \infty}\left\|z_{n}-S z_{n}\right\|=0 .
$$

Step 4. We prove that $x_{n} \rightarrow x^{*}=P_{\Omega} x_{0}$ as $n \rightarrow \infty$.

Indeed, since $\left\{x_{n}\right\}$ is bounded, there exists a subsequence $\left\{x_{n_{i}}\right\}$ which converges weakly to some $w$. From (78), (84), (103), and (101), we have that $z_{n_{i}} \rightarrow w, u_{n_{i}} \rightarrow w, v_{n_{i}} \rightarrow w$ and $\Lambda_{n_{i}}^{m} u_{n_{i}} \rightarrow w$, where $m \in\{1,2, \ldots, N\}$. Since $S$ is uniformly 
continuous, by (128) we get $\lim _{n \rightarrow \infty}\left\|z_{n}-S^{m} z_{n}\right\|=0$ for any $m \geq 1$. Hence from Lemma 24, we obtain $w \in \operatorname{Fix}(S)$. In the meantime, utilizing Lemma 12, we deduce from $v_{n_{i}} \rightarrow w$, $x_{n_{i}} \rightarrow w$, (118), and (123) that $w \in \operatorname{SGEP}(G)$ and $w \in$ $\operatorname{Fix}\left(P_{C}(I-(2 / L) \nabla f)\right)=V I(C, \nabla f)=\Gamma$. Next we prove that $w \in \cap_{m=1}^{N} I\left(B_{m}, R_{m}\right)$. As a matter of fact, since $B_{m}$ is $\eta_{m^{-}}$ inverse strongly monotone, $B_{m}$ is a monotone and Lipschitz continuous mapping. It follows from Lemma 20 that $R_{m}+B_{m}$ is maximal monotone. Let $(v, g) \in G\left(R_{m}+B_{m}\right)$; that is, $g-$ $B_{m} v \in R_{m} v$. Again, since $\Lambda_{n}^{m} u_{n}=J_{R_{m}, \lambda_{m, n}}\left(I-\lambda_{m, n} B_{m}\right) \Lambda_{n}^{m-1} u_{n}$, $n \geq 1, m \in\{1,2, \ldots, N\}$, we have

$$
\Lambda_{n}^{m-1} u_{n}-\lambda_{m, n} B_{m} \Lambda_{n}^{m-1} u_{n} \in\left(I+\lambda_{m, n} R_{m}\right) \Lambda_{n}^{m} u_{n}
$$

that is,

$$
\frac{1}{\lambda_{m, n}}\left(\Lambda_{n}^{m-1} u_{n}-\Lambda_{n}^{m} u_{n}-\lambda_{m, n} B_{m} \Lambda_{n}^{m-1} u_{n}\right) \in R_{m} \Lambda_{n}^{m} u_{n}
$$

In terms of the monotonicity of $R_{m}$, we get

$$
\begin{aligned}
& \left\langle v-\Lambda_{n}^{m} u_{n}, g-B_{m} v-\frac{1}{\lambda_{m, n}}\right. \\
& \left.\quad \times\left(\Lambda_{n}^{m-1} u_{n}-\Lambda_{n}^{m} u_{n}-\lambda_{m, n} B_{m} \Lambda_{n}^{m-1} u_{n}\right)\right\rangle \geq 0,
\end{aligned}
$$

and hence

$$
\begin{aligned}
\left\langle v-\Lambda_{n}^{m} u_{n}, g\right\rangle & \left\langle v-\Lambda_{n}^{m} u_{n}, B_{m} v+\frac{1}{\lambda_{m, n}}\right. \\
\geq & \left.\times\left(\Lambda_{n}^{m-1} u_{n}-\Lambda_{n}^{m} u_{n}-\lambda_{m, n} B_{m} \Lambda_{n}^{m-1} u_{n}\right)\right\rangle \\
= & \left\langle v-\Lambda_{n}^{m} u_{n}, B_{m} v-B_{m} \Lambda_{n}^{m} u_{n}+B_{m} \Lambda_{n}^{m} u_{n}\right. \\
& \left.\quad-B_{m} \Lambda_{n}^{m-1} u_{n}+\frac{1}{\lambda_{m, n}}\left(\Lambda_{n}^{m-1} u_{n}-\Lambda_{n}^{m} u_{n}\right)\right\rangle \\
\geq & \left\langle v-\Lambda_{n}^{m} u_{n}, B_{m} \Lambda_{n}^{m} u_{n}-B_{m} \Lambda_{n}^{m-1} u_{n}\right\rangle \\
& +\left\langle v-\Lambda_{n}^{m} u_{n}, \frac{1}{\lambda_{m, n}}\left(\Lambda_{n}^{m-1} u_{n}-\Lambda_{n}^{m} u_{n}\right)\right\rangle .
\end{aligned}
$$

In particular,

$$
\begin{aligned}
\langle v- & \left.\Lambda_{n_{i}}^{m} u_{n_{i}}, g\right\rangle \\
\geq & \left\langle v-\Lambda_{n_{i}}^{m} u_{n_{i}}, B_{m} \Lambda_{n_{i}}^{m} u_{n_{i}}-B_{m} \Lambda_{n_{i}}^{m-1} u_{n_{i}}\right\rangle \\
& +\left\langle v-\Lambda_{n_{i}}^{m} u_{n_{i}}, \frac{1}{\lambda_{m, n_{i}}}\left(\Lambda_{n_{i}}^{m-1} u_{n_{i}}-\Lambda_{n_{i}}^{m} u_{n_{i}}\right)\right\rangle .
\end{aligned}
$$

Since $\left\|\Lambda_{n}^{m} u_{n}-\Lambda_{n}^{m-1} u_{n}\right\| \rightarrow 0$ (due to (90)) and $\| B_{m} \Lambda_{n}^{m} u_{n}-$ $B_{m} \Lambda_{n}^{m-1} u_{n} \| \rightarrow 0$ (due to the Lipschitz continuity of $B_{m}$ ), we conclude from $\Lambda_{n_{i}}^{m} u_{n_{i}} \rightarrow w$ and $\left\{\lambda_{m, n}\right\} \subset\left[a_{m}, b_{m}\right] \subset$ $\left(0,2 \eta_{m}\right)$ that

$$
\lim _{i \rightarrow \infty}\left\langle v-\Lambda_{n_{i}}^{m} u_{n_{i}}, g\right\rangle=\langle v-w, g\rangle \geq 0
$$

It follows from the maximal monotonicity of $B_{m}+R_{m}$ that $0 \in\left(R_{m}+B_{m}\right) w$; that is, $w \in I\left(B_{m}, R_{m}\right)$. Therefore, $w \in$ $\cap_{m=1}^{N} I\left(B_{m}, R_{m}\right)$.

Next, we show that $w \in \operatorname{GMEP}(\Theta, \varphi, A)$. In fact, from $u_{n}=S_{r_{n}}^{(\Theta, \varphi)}\left(I-r_{n} A\right) x_{n}$, we know that

$$
\begin{aligned}
& \Theta\left(u_{n}, y\right)+\varphi(y)-\varphi\left(u_{n}\right)+\left\langle A x_{n}, y-u_{n}\right\rangle \\
& +\frac{1}{r_{n}}\left\langle K^{\prime}\left(u_{n}\right)-K^{\prime}\left(x_{n}\right), y-u_{n}\right\rangle \geq 0, \quad \forall y \in C .
\end{aligned}
$$

From (H2) it follows that

$$
\begin{aligned}
\varphi(y) & -\varphi\left(u_{n}\right)+\left\langle A x_{n}, y-u_{n}\right\rangle \\
& +\frac{1}{r_{n}}\left\langle K^{\prime}\left(u_{n}\right)-K^{\prime}\left(x_{n}\right), y-u_{n}\right\rangle \\
\geq & \Theta\left(y, u_{n}\right), \quad \forall y \in C .
\end{aligned}
$$

Replacing $n$ by $n_{i}$, we have

$$
\begin{aligned}
& \varphi(y)-\varphi\left(u_{n_{i}}\right)+\left\langle A x_{n_{i}}, y-u_{n_{i}}\right\rangle \\
&+\left\langle\frac{K^{\prime}\left(u_{n_{i}}\right)-K^{\prime}\left(x_{n_{i}}\right)}{r_{n_{i}}}, y-u_{n_{i}}\right\rangle \geq \Theta\left(y, u_{n_{i}}\right), \\
& \forall y \in C .
\end{aligned}
$$

Put $u_{t}=t y+(1-t) w$ for all $t \in(0,1]$ and $y \in C$. Then, from (137) we have

$$
\begin{aligned}
\left\langle u_{t}-\right. & \left.u_{n_{i}}, A u_{t}\right\rangle \\
\geq & \left\langle u_{t}-u_{n_{i}}, A u_{t}\right\rangle-\varphi\left(u_{t}\right)+\varphi\left(u_{n_{i}}\right) \\
& -\left\langle u_{t}-u_{n_{i}}, A x_{n_{i}}\right\rangle-\left\langle\frac{K^{\prime}\left(u_{n_{i}}\right)-K^{\prime}\left(x_{n_{i}}\right)}{r_{n_{i}}}, u_{t}-u_{n_{i}}\right\rangle \\
& +\Theta\left(u_{t}, u_{n_{i}}\right) \\
\geq & \left\langle u_{t}-u_{n_{i}}, A u_{t}-A u_{n_{i}}\right\rangle+\left\langle u_{t}-u_{n_{i}}, A u_{n_{i}}-A x_{n_{i}}\right\rangle \\
& -\varphi\left(u_{t}\right)+\varphi\left(u_{n_{i}}\right) \\
& -\left\langle\frac{K^{\prime}\left(u_{n_{i}}\right)-K^{\prime}\left(x_{n_{i}}\right)}{r_{n_{i}}}, u_{t}-u_{n_{i}}\right\rangle+\Theta\left(u_{t}, u_{n_{i}}\right) .
\end{aligned}
$$

Since $\left\|u_{n_{i}}-x_{n_{i}}\right\| \rightarrow 0$ as $i \rightarrow \infty$, we deduce from the Lipschitz continuity of $A$ and $K^{\prime}$ that $\left\|A u_{n_{i}}-A x_{n_{i}}\right\| \rightarrow 0$ and $\left\|K^{\prime}\left(u_{n_{i}}\right)-K^{\prime}\left(x_{n_{i}}\right)\right\| \rightarrow 0$ as $i \rightarrow \infty$. Further, from the monotonicity of $A$, we have $\left\langle u_{t}-u_{n_{i}}, A u_{t}-A u_{n_{i}}\right\rangle \geq 0$. So, 
from (H4), the weakly lower semicontinuity of $\varphi,\left(K^{\prime}\left(u_{n_{i}}\right)-\right.$ $\left.K^{\prime}\left(x_{n_{i}}\right)\right) / r_{n_{i}} \rightarrow 0$, and $u_{n_{i}} \rightarrow w$, we have

$$
\left\langle u_{t}-w, A u_{t}\right\rangle \geq-\varphi\left(u_{t}\right)+\varphi(w)+\Theta\left(u_{t}, w\right), \quad \text { as } i \longrightarrow \infty .
$$

From (H1), (H4), and (139) we also have

$$
\begin{aligned}
0= & \Theta\left(u_{t}, u_{t}\right)+\varphi\left(u_{t}\right)-\varphi\left(u_{t}\right) \\
\leq & t \Theta\left(u_{t}, y\right)+(1-t) \Theta\left(u_{t}, w\right) \\
& +t \varphi(y)+(1-t) \varphi(w)-\varphi\left(u_{t}\right) \\
= & t\left[\Theta\left(u_{t}, y\right)+\varphi(y)-\varphi\left(u_{t}\right)\right] \\
& +(1-t)\left[\Theta\left(u_{t}, w\right)+\varphi(w)-\varphi(w)-\varphi\left(u_{t}\right)\right] \\
\leq & t\left[\Theta\left(u_{t}, y\right)+\varphi(y)-\varphi\left(u_{t}\right)\right]+(1-t)\left\langle u_{t}-w, A u_{t}\right\rangle \\
= & t\left[\Theta\left(u_{t}, y\right)+\varphi(y)-\varphi\left(u_{t}\right)\right]+(1-t) t\left\langle y-w, A u_{t}\right\rangle,
\end{aligned}
$$

and hence

$$
0 \leq \Theta\left(u_{t}, y\right)+\varphi(y)-\varphi\left(u_{t}\right)+(1-t)\left\langle y-w, A u_{t}\right\rangle .
$$

Letting $t \rightarrow 0$, we have, for each $y \in C$,

$$
0 \leq \Theta(w, y)+\varphi(y)-\varphi(w)+\langle A w, y-w\rangle
$$

This implies that $w \in \operatorname{GMEP}(\Theta, \varphi, A)$. Consequently, $w \in$ $\Omega=\operatorname{GMEP}(\Theta, \varphi, A) \cap \operatorname{SGEP}(G) \cap\left(\cap_{i=1}^{N} I\left(B_{i}, R_{i}\right)\right) \cap \operatorname{Fix}(S) \cap \Gamma$. This shows that $\omega_{w}\left(x_{n}\right) \subset \Omega$. From (65) and Lemma 15 we infer that $x_{n} \rightarrow x^{*}=P_{\Omega} x_{0}$ as $n \rightarrow \infty$.

Finally, assume additionally that $\left\|x_{n}-z_{n}\right\|=o\left(s_{n}\right)$. It is clear that

$$
\begin{array}{r}
\langle(V-\gamma Q) x-(V-\gamma Q) y, x-y\rangle \geq(\bar{\gamma}-\gamma l)\|x-y\|^{2}, \\
\forall x, y \in H .
\end{array}
$$

So, we know that $V-\gamma Q$ is $(\bar{\gamma}-\gamma l)$-strongly monotone with constant $\bar{\gamma}-\gamma l>0$. In the meantime, it is easy to see that $V-\gamma Q$ is $(\|V\|+\gamma l)$-Lipschitzian with constant $\|V\|+\gamma l>0$. Thus, there exists a unique solution $\widehat{x}$ in $\Omega$ to the VIP

$$
\langle(\gamma Q-V) \hat{x}, p-\widehat{x}\rangle \leq 0, \quad \forall p \in \Omega
$$

Equivalently, $\widehat{x}=P_{\Omega}(I-V+\gamma Q) \hat{x}$. Furthermore, from (59), (60), and (83) we get

$$
\begin{aligned}
&\left\|z_{n}-p\right\|^{2} \\
& \leq {\left[\left(\beta_{n}+s_{n} \bar{\gamma}\right)\left\|x_{n}-p\right\|+\left(1-\beta_{n}-s_{n} \bar{\gamma}\right)\left\|G v_{n}-p\right\|\right]^{2} } \\
&+2 s_{n}\left\langle(\gamma Q-V) p, z_{n}-p\right\rangle \\
& \leq\left(\beta_{n}+s_{n} \bar{\gamma}\right)\left\|x_{n}-p\right\|^{2}+\left(1-\beta_{n}-s_{n} \bar{\gamma}\right)\left\|G v_{n}-p\right\|^{2} \\
&+2 s_{n}\left\langle(\gamma Q-V) p, z_{n}-p\right\rangle \\
& \leq\left(\beta_{n}+s_{n} \bar{\gamma}\right)\left\|x_{n}-p\right\|^{2}+\left(1-\beta_{n}-s_{n} \bar{\gamma}\right)\left\|v_{n}-p\right\|^{2} \\
&+2 s_{n}\left\langle(\gamma Q-V) p, z_{n}-p\right\rangle \\
& \leq\left(\beta_{n}+s_{n} \bar{\gamma}\right)\left\|x_{n}-p\right\|^{2}+\left(1-\beta_{n}-s_{n} \bar{\gamma}\right)\left\|x_{n}-p\right\|^{2} \\
&+2 s_{n}\left\langle(\gamma Q-V) p, z_{n}-p\right\rangle \\
&=\left\|x_{n}-p\right\|^{2}+2 s_{n}\left\langle(\gamma Q-V) p, z_{n}-p\right\rangle,
\end{aligned}
$$

which hence yields

$$
\begin{aligned}
\left\langle(\gamma Q-V) p, p-z_{n}\right\rangle & \leq \frac{\left\|x_{n}-p\right\|^{2}-\left\|z_{n}-p\right\|^{2}}{2 s_{n}} \\
& \leq \frac{\left\|x_{n}-z_{n}\right\|}{2 s_{n}}\left(\left\|x_{n}-p\right\|+\left\|z_{n}-p\right\|\right) .
\end{aligned}
$$

Since $\left\|x_{n}-z_{n}\right\|=o\left(s_{n}\right), \lim _{n \rightarrow \infty}\left\|x_{n}-x^{*}\right\|=0$, and $\left\{x_{n}\right\},\left\{z_{n}\right\}$ are bounded, we infer from (146) that

$$
\left\langle(\gamma Q-V) p, p-x^{*}\right\rangle \leq 0, \quad \forall p \in \Omega,
$$

which together with Minty's lemma [4] implies that

$$
\left\langle(\gamma Q-V) x^{*}, p-x^{*}\right\rangle \leq 0, \quad \forall p \in \Omega .
$$

This shows that $x^{*}$ is a solution in $\Omega$ to the VIP (144). Utilizing the uniqueness of solutions in $\Omega$ to the VIP (144), we get $x^{*}=$ $\widehat{x}$. This completes the proof.

Corollary 27. Let $C$ be a nonempty closed convex subset of a real Hilbert space $H$. Let $f: C \rightarrow \mathbf{R}$ be a convex functional with L-Lipschitz continuous gradient $\nabla f$. Let $\Theta, \Theta_{1}, \Theta_{2}$ be three bifunctions from $\mathrm{C} \times \mathrm{C}$ to $\mathbf{R}$ satisfying $(\mathrm{H1})-(\mathrm{H} 4)$ and $\varphi: C \rightarrow \mathbf{R}$ a lower semicontinuous and convex functional. Let $R_{i}: C \rightarrow 2^{H}$ be a maximal monotone mapping and let $A, A_{k}: H \rightarrow H$ and $B_{i}: C \rightarrow H$ be $\zeta$ inverse strongly monotone, $\zeta_{k}$-inverse strongly monotone, and $\eta_{i}$-inverse-strongly monotone, respectively, for $k=1,2$ and $i=1$, 2. Let $S: C \rightarrow C$ be a uniformly continuous asymptotically $k$-strict pseudocontractive mapping in the intermediate sense for some $0 \leq k<1$ with sequence $\left\{\gamma_{n}\right\} \subset[0, \infty)$ such that 
$\lim _{n \rightarrow \infty} \gamma_{n}=0$ and $\left\{c_{n}\right\} \subset[0, \infty)$ such that $\lim _{n \rightarrow \infty} c_{n}=0$. Let $V$ be a $\bar{\gamma}$-strongly positive bounded linear operator and $Q: H \rightarrow H$ an l-Lipschitzian mapping with $\gamma l<\bar{\gamma}$. Assume that $\Omega:=\operatorname{GMEP}(\Theta, \varphi, A) \cap \operatorname{SGEP}(G) \cap I\left(B_{1}, R_{1}\right) \cap I\left(B_{2}, R_{2}\right) \cap$ $\operatorname{Fix}(S) \cap \Gamma$ is nonempty and bounded where $G$ is defined as in Proposition CY. Let $\left\{r_{n}\right\}$ be a sequence in $[0,2 \zeta]$ and $\left\{\alpha_{n}\right\},\left\{\beta_{n}\right\}$, and $\left\{\delta_{n}\right\}$ be sequences in $[0,1]$ such that $0<\alpha \leq \alpha_{n} \leq 1$ and $k \leq \delta_{n} \leq d<1$, and let $\nu_{k} \in\left(0,2 \zeta_{k}\right), k=1,2$ and $\left\{\lambda_{i, n}\right\} \subset\left[a_{i}, b_{i}\right] \subset\left(0,2 \eta_{i}\right), i=1,2$. Pick any $x_{0} \in H$ and set $C_{1}=C, x_{1}=P_{C_{1}} x_{0}$. Let $\left\{x_{n}\right\}$ be a sequence generated by the following algorithm:

$$
\begin{aligned}
& u_{n}=S_{r_{n}}^{(\Theta, \varphi)}\left(I-r_{n} A\right) x_{n}, \\
& v_{n}=J_{R_{2}, \lambda_{2, n}}\left(I-\lambda_{2, n} B_{2}\right) J_{R_{1}, \lambda_{1, n}}\left(I-\lambda_{1, n} B_{1}\right) u_{n}, \\
& z_{n}=s_{n} \gamma Q x_{n}+\beta_{n} x_{n}+\left(\left(1-\beta_{n}\right) I-s_{n} V\right) T_{n} G v_{n}, \\
& k_{n}=\delta_{n} z_{n}+\left(1-\delta_{n}\right) S^{n} z_{n}, \\
& y_{n}=\left(1-\alpha_{n}\right) x_{n}+\alpha_{n} k_{n}, \\
& C_{n+1}=\left\{z \in C_{n}:\left\|y_{n}-z\right\|^{2} \leq\left\|x_{n}-z\right\|^{2}+\theta_{n}\right\}, \\
& x_{n+1}=P_{C_{n+1}} x_{0}, \forall n \geq 1,
\end{aligned}
$$

where $P_{C}\left(I-\lambda_{n} \nabla f\right)=s_{n} I+\left(1-s_{n}\right) T_{n}$ (here $T_{n}$ is nonexpansive, $s_{n}=\left(2-\lambda_{n} L\right) / 4 \in(0,1 / 2)$ for each $\left.\lambda_{n} \in(0,2 / L)\right)$, and $\theta_{n}=\left(s_{n}+\gamma_{n}\right)\left(1+\gamma_{n}\right) \Delta_{n}+c_{n}, \Delta_{n}=\sup \left\{\left\|x_{n}-p\right\|^{2}+\right.$ $\left.\|(\gamma Q-V) p\|^{2} /(\bar{\gamma}-\gamma l): p \in \Omega\right\}<\infty$. Assume that the following conditions are satisfied:

(i) $K: H \rightarrow \mathbf{R}$ is strongly convex with constant $\sigma>0$ and its derivative $K^{\prime}$ is Lipschitz continuous with constant $v>0$ such that the function $x \mapsto\left\langle y-x, K^{\prime}(x)\right\rangle$ is weakly upper semicontinuous for each $y \in H$;

(ii) for each $x \in H$, there exist a bounded subset $D_{x} \subset C$ and $z_{x} \in C$ such that for any $y \notin D_{x}$,

$$
\begin{aligned}
& \Theta\left(y, z_{x}\right)+\varphi\left(z_{x}\right)-\varphi(y) \\
& +\frac{1}{r}\left\langle K^{\prime}(y)-K^{\prime}(x), z_{x}-y\right\rangle<0 ;
\end{aligned}
$$

(iii) $s_{n} \in(0,1 / 2)$ for each $\lambda_{n} \in(0,2 / L)$, and $\lim _{n \rightarrow \infty} s_{n}=$ $0\left(\Leftrightarrow \lim _{n \rightarrow \infty} \lambda_{n}=2 / L\right) ;$

(iv) $0<\liminf _{n \rightarrow \infty} \beta_{n} \leq \limsup _{n \rightarrow \infty} \beta_{n}<1$ and $0<$ $\liminf _{n \rightarrow \infty} r_{n} \leq \limsup _{n \rightarrow \infty} r_{n}<2 \zeta$.

Assume that $S_{r}^{(\Theta, \varphi)}$ is firmly nonexpansive. Then we have

(i) $\left\{x_{n}\right\}$ converges strongly as $\lambda_{n} \rightarrow(2 / L)\left(\Leftrightarrow s_{n} \rightarrow 0\right)$ to $x^{*}=P_{\Omega} x_{0}$;

(ii) $\left\{x_{n}\right\}$ converges strongly as $\lambda_{n} \rightarrow(2 / L)\left(\Leftrightarrow s_{n} \rightarrow 0\right)$ to $x^{*}=P_{\Omega} x_{0}$ provided that $\left\|x_{n}-z_{n}\right\|=o\left(s_{n}\right)$, which is the unique solution in $\Omega$ to the VIP

$$
\left\langle(\gamma Q-V) x^{*}, p-x^{*}\right\rangle \leq 0, \quad \forall p \in \Omega .
$$

Equivalently, $x^{*}=P_{\Omega}(I-V+\gamma Q) x^{*}$.
Corollary 28. Let $C$ be a nonempty closed convex subset of a real Hilbert space $H$. Let $f: C \rightarrow \mathbf{R}$ be a convex functional with L-Lipschitz continuous gradient $\nabla f$. Let $\Theta, \Theta_{1}, \Theta_{2}$ be three bifunctions from $\mathrm{C} \times \mathrm{C}$ to $\mathbf{R}$ satisfying $(\mathrm{H1})-(\mathrm{H} 4)$ and $\varphi$ : $C \rightarrow \mathbf{R}$ a lower semicontinuous and convex functional. Let $R: C \rightarrow 2^{H}$ be a maximal monotone mapping and let $A, A_{k}$ : $H \rightarrow H$ and $B: C \rightarrow H$ be $\zeta$-inverse strongly monotone, $\zeta_{k^{-}}$ inverse strongly monotone, and $\xi$-inverse-strongly monotone, respectively, for $k=1,2$. Let $S: C \rightarrow C$ be a uniformly continuous asymptotically $k$-strict pseudocontractive mapping in the intermediate sense for some $0 \leq k<1$ with sequence $\left\{\gamma_{n}\right\} \subset[0, \infty)$ such that $\lim _{n \rightarrow \infty} \gamma_{n}=0$ and $\left\{c_{n}\right\} \subset[0, \infty)$ such that $\lim _{n \rightarrow \infty} c_{n}=0$. Let $V$ be a $\bar{\gamma}$-strongly positive bounded linear operator and $Q: H \rightarrow H$ be an l-Lipschitzian mapping with $\gamma l<\bar{\gamma}$. Assume that $\Omega:=\operatorname{GMEP}(\Theta, \varphi, A) \cap \operatorname{SGEP}(G) \cap$ $I(B, R) \cap \operatorname{Fix}(S) \cap \Gamma$ is nonempty and bounded where $G$ is defined as in Proposition CY. Let $\left\{r_{n}\right\}$ be a sequence in $[0,2 \zeta]$ and $\left\{\alpha_{n}\right\},\left\{\beta_{n}\right\}$ and $\left\{\delta_{n}\right\}$ be sequences in $[0,1]$ such that $0<\alpha \leq$ $\alpha_{n} \leq 1$ and $k \leq \delta_{n} \leq d<1$, and let $\nu_{k} \in\left(0,2 \zeta_{k}\right), k=1,2$ and $\left\{\rho_{n}\right\} \subset[a, b] \subset(0,2 \xi)$. Pick any $x_{0} \in H$ and set $C_{1}=C$, $x_{1}=P_{C_{1}} x_{0}$. Let $\left\{x_{n}\right\}$ be a sequence generated by the following algorithm:

$$
\begin{aligned}
u_{n} & =S_{r_{n}}^{(\Theta, \varphi)}\left(I-r_{n} A\right) x_{n}, \\
v_{n} & =J_{R, \rho_{n}}\left(I-\rho_{n} B\right) u_{n}, \\
z_{n} & =s_{n} \gamma Q x_{n}+\beta_{n} x_{n}+\left(\left(1-\beta_{n}\right) I-s_{n} V\right) T_{n} G v_{n}, \\
k_{n} & =\delta_{n} z_{n}+\left(1-\delta_{n}\right) S^{n} z_{n}, \\
y_{n} & =\left(1-\alpha_{n}\right) x_{n}+\alpha_{n} k_{n}, \\
C_{n+1} & =\left\{z \in C_{n}:\left\|y_{n}-z\right\|^{2} \leq\left\|x_{n}-z\right\|^{2}+\theta_{n}\right\}, \\
x_{n+1} & =P_{C_{n+1}} x_{0}, \quad \forall n \geq 1,
\end{aligned}
$$

where $P_{C}\left(I-\lambda_{n} \nabla f\right)=s_{n} I+\left(1-s_{n}\right) T_{n}$ (here $T_{n}$ is nonexpansive, $s_{n}=\left(2-\lambda_{n} L\right) / 4 \in(0,1 / 2)$ for each $\left.\lambda_{n} \in(0,2 / L)\right)$ and $\theta_{n}=\left(s_{n}+\gamma_{n}\right)\left(1+\gamma_{n}\right) \Delta_{n}+c_{n}, \Delta_{n}=\sup \left\{\left\|x_{n}-p\right\|^{2}+\right.$ $\left.\|(\gamma Q-V) p\|^{2} /(\bar{\gamma}-\gamma l): p \in \Omega\right\}<\infty$. Assume that the following conditions are satisfied:

(i) $K: H \rightarrow \mathbf{R}$ is strongly convex with constant $\sigma>0$ and its derivative $K^{\prime}$ is Lipschitz continuous with constant $v>0$ such that the function $x \mapsto\left\langle y-x, K^{\prime}(x)\right\rangle$ is weakly upper semicontinuous for each $y \in H$;

(ii) for each $x \in H$, there exist a bounded subset $D_{x} \subset C$ and $z_{x} \in C$ such that for any $y \notin D_{x}$,

$$
\Theta\left(y, z_{x}\right)+\varphi\left(z_{x}\right)-\varphi(y)+\frac{1}{r}\left\langle K^{\prime}(y)-K^{\prime}(x), z_{x}-y\right\rangle
$$$$
<0
$$

(iii) $s_{n} \in(0,1 / 2)$ for each $\lambda_{n} \in(0,2 / L)$, and $\lim _{n \rightarrow \infty} s_{n}=$ $0\left(\Leftrightarrow \lim _{n \rightarrow \infty} \lambda_{n}=(2 / L)\right) ;$

(iv) $0<\liminf _{n \rightarrow \infty} \beta_{n} \leq \limsup _{n \rightarrow \infty} \beta_{n}<1$ and $0<$ $\liminf _{n \rightarrow \infty} r_{n} \leq \lim \sup _{n \rightarrow \infty} r_{n}<2 \zeta$. 
Assume that $S_{r}^{(\Theta, \varphi)}$ is firmly nonexpansive. Then we have

(i) $\left\{x_{n}\right\}$ converges strongly as $\lambda_{n} \rightarrow(2 / L)\left(\Leftrightarrow s_{n} \rightarrow 0\right)$ to $x^{*}=P_{\Omega} x_{0}$;

(ii) $\left\{x_{n}\right\}$ converges strongly as $\lambda_{n} \rightarrow(2 / L)\left(\Leftrightarrow s_{n} \rightarrow 0\right)$ to $x^{*}=P_{\Omega} x_{0}$ provided that $\left\|x_{n}-z_{n}\right\|=o\left(s_{n}\right)$, which is the unique solution in $\Omega$ to the VIP

$$
\left\langle(\gamma Q-V) x^{*}, p-x^{*}\right\rangle \leq 0, \quad \forall p \in \Omega
$$

Equivalently, $x^{*}=P_{\Omega}(I-V+\gamma Q) x^{*}$.

Corollary 29. Let $C$ be a nonempty closed convex subset of a real Hilbert space $H$. Let $f: C \rightarrow \mathbf{R}$ be a convex functional with L-Lipschitz continuous gradient $\nabla f$. Let $\Theta, \Theta_{1}, \Theta_{2}$ be three bifunctions from $\mathrm{C} \times \mathrm{C}$ to $\mathbf{R}$ satisfying $(\mathrm{H} 1)-(\mathrm{H} 4)$ and $\varphi: C \rightarrow \mathbf{R}$ a lower semicontinuous and convex functional. Let $R: C \rightarrow 2^{H}$ be a maximal monotone mapping and let $A, A_{k}$ : $H \rightarrow H$ and $B: C \rightarrow H$ be $\zeta$-inverse strongly monotone, $\zeta_{k^{-}}$ inverse strongly monotone, and $\xi$-inverse-strongly monotone, respectively, for $k=1,2$. Let $S: C \rightarrow C$ be a uniformly continuous asymptotically $k$-strict pseudocontractive mapping for some $0 \leq k<1$ with sequence $\left\{\gamma_{n}\right\} \subset[0, \infty)$ such that $\lim _{n \rightarrow \infty} \gamma_{n}=0$. Let $V$ be a $\bar{\gamma}$-strongly positive bounded linear operator and $Q: H \rightarrow H$ anl-Lipschitzian mapping with $\gamma l<$ $\bar{\gamma}$. Assume that $\Omega:=\operatorname{GMEP}(\Theta, \varphi, A) \cap \operatorname{SGEP}(G) \cap I(B, R) \cap$ $\operatorname{Fix}(S) \cap \Gamma$ is nonempty and bounded where $G$ is defined as in Proposition CY. Let $\left\{r_{n}\right\}$ be a sequence in $[0,2 \zeta]$ and $\left\{\alpha_{n}\right\},\left\{\beta_{n}\right\}$, and $\left\{\delta_{n}\right\}$ be sequences in $[0,1]$ such that $0<\alpha \leq \alpha_{n} \leq 1$ and $k \leq \delta_{n} \leq d<1$, and let $\nu_{k} \in\left(0,2 \zeta_{k}\right), k=1,2$ and $\left\{\rho_{n}\right\} \subset$ $[a, b] \subset(0,2 \xi)$. Pick any $x_{0} \in H$ and set $C_{1}=C, x_{1}=P_{C_{1}} x_{0}$. Let $\left\{x_{n}\right\}$ be a sequence generated by the following algorithm:

$$
\begin{aligned}
u_{n} & =S_{r_{n}}^{(\Theta, \varphi)}\left(I-r_{n} A\right) x_{n}, \\
v_{n} & =J_{R, \rho_{n}}\left(I-\rho_{n} B\right) u_{n}, \\
z_{n} & =s_{n} \gamma Q x_{n}+\beta_{n} x_{n}+\left(\left(1-\beta_{n}\right) I-s_{n} V\right) T_{n} G v_{n}, \\
k_{n} & =\delta_{n} z_{n}+\left(1-\delta_{n}\right) S^{n} z_{n}, \\
y_{n} & =\left(1-\alpha_{n}\right) x_{n}+\alpha_{n} k_{n}, \\
C_{n+1} & =\left\{z \in C_{n}:\left\|y_{n}-z\right\|^{2} \leq\left\|x_{n}-z\right\|^{2}+\theta_{n}\right\}, \\
x_{n+1} & =P_{C_{n+1}} x_{0}, \quad \forall n \geq 1,
\end{aligned}
$$

where $P_{C}\left(I-\lambda_{n} \nabla f\right)=s_{n} I+\left(1-s_{n}\right) T_{n}$ (here $T_{n}$ is nonexpansive, $s_{n}=\left(2-\lambda_{n} L\right) / 4 \in(0,1 / 2)$ for each $\left.\lambda_{n} \in(0,2 / L)\right)$, and $\theta_{n}=$ $\left(s_{n}+\gamma_{n}\right)\left(1+\gamma_{n}\right) \Delta_{n}, \Delta_{n}=\sup \left\{\left\|x_{n}-p\right\|^{2}+\|(\gamma Q-V) p\|^{2} /(\bar{\gamma}-\right.$ $\gamma l): p \in \Omega\}<\infty$. Assume that the following conditions are satisfied:

(i) $K: H \rightarrow \mathbf{R}$ is strongly convex with constant $\sigma>0$ and its derivative $K^{\prime}$ is Lipschitz continuous with constant $v>0$ such that the function $x \mapsto\left\langle y-x, K^{\prime}(x)\right\rangle$ is weakly upper semicontinuous for each $y \in H$; (ii) for each $x \in H$, there exist a bounded subset $D_{x} \subset C$ and $z_{x} \in C$ such that for any $y \notin D_{x}$,

$\Theta\left(y, z_{x}\right)+\varphi\left(z_{x}\right)-\varphi(y)+\frac{1}{r}\left\langle K^{\prime}(y)-K^{\prime}(x), z_{x}-y\right\rangle<0 ;$

(iii) $s_{n} \in(0,1 / 2)$ for each $\lambda_{n} \in(0,2 / L)$, and $\lim _{n \rightarrow \infty} s_{n}=$ $0\left(\Leftrightarrow \lim _{n \rightarrow \infty} \lambda_{n}=2 / L\right) ;$

(iv) $0<\liminf _{n \rightarrow \infty} \beta_{n} \leq \limsup _{n \rightarrow \infty} \beta_{n}<1$ and $0<$ $\liminf _{n \rightarrow \infty} r_{n} \leq \limsup _{n \rightarrow \infty} r_{n}<2 \zeta$.

Assume that $S_{r}^{(\Theta, \varphi)}$ is firmly nonexpansive. Then we have

(i) $\left\{x_{n}\right\}$ converges strongly as $\lambda_{n} \rightarrow(2 / L)\left(\Leftrightarrow s_{n} \rightarrow 0\right)$ to $x^{*}=P_{\Omega} x_{0}$;

(ii) $\left\{x_{n}\right\}$ converges strongly as $\lambda_{n} \rightarrow(2 / L)\left(\Leftrightarrow s_{n} \rightarrow 0\right)$ to $x^{*}=P_{\Omega} x_{0}$ provided that $\left\|x_{n}-z_{n}\right\|=o\left(s_{n}\right)$, which is the unique solution in $\Omega$ to the VIP

$$
\left\langle(\gamma Q-V) x^{*}, p-x^{*}\right\rangle \leq 0, \quad \forall p \in \Omega .
$$

Equivalently, $x^{*}=P_{\Omega}(I-V+\gamma Q) x^{*}$.

\section{Weak Convergence Theorem}

In this section, we will introduce and analyze another multistep iterative algorithm involving no shrinking projection method for finding a solution of the SGEP (8) with constraints of several problems: the GMEP (4), the CMP (12), finitely many variational inclusions, and the fixed point problem of an asymptotically strict pseudocontractive mapping in the intermediate sense in a real Hilbert space. We prove weak convergence theorem for the iterative algorithm under mild assumptions. This iterative algorithm is based on Korpelevich's extragradient method, strongly positive bounded linear operator approach, viscosity approximation method, averaged mapping approach to the GPA in [16], and Mann-type iteration method.

Theorem 30. Let $C$ be a nonempty closed convex subset of a real Hilbert space $H$. Let $N$ be an integer. Let $f: C \rightarrow \mathbf{R}$ be a convex functional with L-Lipschitz continuous gradient $\nabla f$. Let $\Theta, \Theta_{1}, \Theta_{2}$ be three bifunctions from $C \times C$ to $\mathbf{R}$ satisfying (H1)-(H4) and $\varphi: C \rightarrow \mathbf{R}$ a lower semicontinuous and convex functional. Let $R_{i}: C \rightarrow 2^{H}$ be a maximal monotone mapping and let $A, A_{k}: H \rightarrow H$ and $B_{i}: C \rightarrow H$ be $\zeta$-inverse strongly monotone, $\zeta_{k}$-inverse strongly monotone and $\eta_{i}$-inverse-strongly monotone, respectively, for $k=1,2$ and $i=1,2, \ldots, N$. Let $S: C \rightarrow C$ be a uniformly continuous asymptotically $k$-strict pseudocontractive mapping in the intermediate sense for some $0 \leq k<1$ with sequence $\left\{\gamma_{n}\right\} \subset[0, \infty)$ such that $\sum_{n=1}^{\infty} \gamma_{n}<\infty$ and $\left\{c_{n}\right\} \subset[0, \infty)$ such that $\sum_{n=1}^{\infty} c_{n}<\infty$. Let $V$ be a $\bar{\gamma}$-strongly positive bounded linear operator and $Q: H \rightarrow H$ an l-Lipschitzian mapping with $\gamma l<\bar{\gamma}$. Assume that $\Omega:=\operatorname{GMEP}(\Theta, \varphi, A) \cap \operatorname{SGEP}(G) \cap$ $\cap_{i=1}^{N} I\left(B_{i}, R_{i}\right) \cap \operatorname{Fix}(S) \cap \Gamma$ is nonempty where $G$ is defined as in Proposition CY. Let $\left\{r_{n}\right\}$ be a sequence in $[0,2 \zeta]$ and $\left\{\alpha_{n}\right\},\left\{\beta_{n}\right\}$ and $\left\{\delta_{n}\right\}$ sequences in $[0,1]$ such that $0<\alpha \leq \alpha_{n} \leq 1$ and 
$0<k+\epsilon \leq \delta_{n} \leq d<1$, and let $\nu_{k} \in\left(0,2 \zeta_{k}\right), k=1,2$ and $\left\{\lambda_{i, n}\right\} \subset\left[a_{i}, b_{i}\right] \subset\left(0,2 \eta_{i}\right), \forall i \in\{1,2, \ldots, N\}$. Pick any $x_{1} \in H$ and let $\left\{x_{n}\right\}$ be a sequence generated by the following algorithm:

$$
\begin{aligned}
u_{n}= & S_{r_{n}}^{(\Theta, \varphi)}\left(I-r_{n} A\right) x_{n}, \\
v_{n}= & J_{R_{N}, \lambda_{N, n}}\left(I-\lambda_{N, n} B_{N}\right) J_{R_{N-1}, \lambda_{N-1, n}} \\
& \times\left(I-\lambda_{N-1, n} A_{N-1}\right) \cdots J_{R_{1}, \lambda_{1, n}}\left(I-\lambda_{1, n} B_{1}\right) u_{n}, \\
z_{n}= & s_{n} \gamma Q x_{n}+\beta_{n} x_{n}+\left(\left(1-\beta_{n}\right) I-s_{n} V\right) T_{n} G v_{n}, \\
k_{n}= & \delta_{n} z_{n}+\left(1-\delta_{n}\right) S^{n} z_{n}, \\
x_{n+1}= & \left(1-\alpha_{n}\right) x_{n}+\alpha_{n} k_{n}, \quad \forall n \geq 1,
\end{aligned}
$$

where $P_{C}\left(I-\lambda_{n} \nabla f\right)=s_{n} I+\left(1-s_{n}\right) T_{n}$ (here $T_{n}$ is nonexpansive, $s_{n}=\left(2-\lambda_{n} L\right) / 4 \in(0,1 / 2)$ for each $\left.\lambda_{n} \in(0,2 / L)\right)$. Assume that the following conditions are satisfied:

(i) $K: H \rightarrow \mathbf{R}$ is strongly convex with constant $\sigma>0$ and its derivative $K^{\prime}$ is Lipschitz continuous with constant $v>0$ such that the function $x \mapsto\left\langle y-x, K^{\prime}(x)\right\rangle$ is weakly upper semicontinuous for each $y \in H$;

(ii) for each $x \in H$, there exist a bounded subset $D_{x} \subset C$ and $z_{x} \in C$ such that for any $y \notin D_{x}$,

$$
\begin{aligned}
& \Theta\left(y, z_{x}\right)+\varphi\left(z_{x}\right)-\varphi(y) \\
& +\frac{1}{r}\left\langle K^{\prime}(y)-K^{\prime}(x), z_{x}-y\right\rangle<0 ;
\end{aligned}
$$

(iii) $s_{n} \in(0,1 / 2)$ for each $\lambda_{n} \in(0,2 / L)$, and $\sum_{n=1}^{\infty} s_{n}<$ $\infty\left(\Leftrightarrow \sum_{n=1}^{\infty}\left(2 / L-\lambda_{n}\right)<\infty\right) ;$

(iv) $0<\lim \inf _{n \rightarrow \infty} \beta_{n} \leq \lim \sup _{n \rightarrow \infty} \beta_{n}<1$ and $0<$ $\lim \inf _{n \rightarrow \infty} r_{n} \leq \lim \sup _{n \rightarrow \infty} r_{n}<2 \zeta$.

Then $\left\{x_{n}\right\}$ converges weakly to $w=\lim _{n \rightarrow \infty} P_{\Omega} x_{n}$ provided that $S_{r}^{(\Theta, \varphi)}$ is firmly nonexpansive.

Proof. Since $\nabla f$ is $L$-Lipschitzian, it follows that $\nabla f$ is $(1 / L)$-ism; see [16]. Repeating the same arguments as in Theorem 26, we can write

$$
P_{C}\left(I-\lambda_{n} \nabla f\right)=\frac{2-\lambda_{n} L}{4} I+\frac{2+\lambda_{n} L}{4} T_{n}=s_{n} I+\left(1-s_{n}\right) T_{n},
$$

where $T_{n}$ is nonexpansive and $s_{n}:=s_{n}\left(\lambda_{n}\right)=\left(2-\lambda_{n} L\right) / 4 \epsilon$ $(0,1 / 2)$ for each $\lambda_{n} \in(0,2 / L)$. It is clear that

$$
\sum_{n=1}^{\infty}\left(\frac{2}{L}-\lambda_{n}\right)<\infty \Longleftrightarrow \sum_{n=1}^{\infty} s_{n}<\infty .
$$

As $\lim _{n \rightarrow \infty} s_{n}=0,0<\lim \inf _{n \rightarrow \infty} \beta_{n} \leq \lim \sup _{n \rightarrow \infty} \beta_{n}<$ 1 and $0<\lim _{\inf }{ }_{n \rightarrow \infty} r_{n} \leq \lim \sup _{n \rightarrow \infty} r_{n}<2 \zeta$, we may assume, without loss of generality, that $\left\{\beta_{n}\right\} \subset[a, \widehat{a}] \subset(0,1)$, $\left\{r_{n}\right\} \subset[c, \widehat{c}] \subset(0,2 \zeta)$ and $\beta_{n}+s_{n}\|V\| \leq 1$ for all $n \geq 1$. Put

$$
\begin{aligned}
\Lambda_{n}^{i}= & J_{R_{i}, \lambda_{i, n}}\left(I-\lambda_{i, n} B_{i}\right) J_{R_{i-1}, \lambda_{i-1, n}} \\
& \times\left(I-\lambda_{i-1, n} B_{i-1}\right) \cdots J_{R_{1}, \lambda_{1, n}}\left(I-\lambda_{1, n} B_{1}\right),
\end{aligned}
$$

for all $i \in\{1,2, \ldots, N\}$, and $\Lambda_{n}^{0}=I$, where $I$ is the identity mapping on $H$. Then we have $v_{n}=\Lambda_{n}^{N} u_{n}$.

Take a fixed $p \in \Omega$ arbitrarily. Let us show the existence of $\lim _{n \rightarrow \infty}\left\|x_{n}-p\right\|$. Indeed, repeating the same arguments as in the proof of Theorem 26, we can obtain that

$$
\begin{gathered}
\left\|\left(1-\beta_{n}\right) I-s_{n} V\right\| \leq 1-\beta_{n}-s_{n} \bar{\gamma} \\
\left\|u_{n}-p\right\|^{2} \leq\left\|x_{n}-p\right\|^{2}+r_{n}\left(r_{n}-2 \zeta\right) \\
\times\left\|A x_{n}-A p\right\|^{2} \leq\left\|x_{n}-p\right\|^{2} \\
\left\|v_{n}-p\right\| \leq\left\|u_{n}-p\right\|, \\
\left.\quad+v_{1}^{2} \leq T_{v_{2}}^{\Theta_{2}}\left(I-v_{2} A_{2}\right) v_{n}-T_{v_{2}}^{\Theta_{2}}\left(I-v_{2} A_{2}\right) p \|^{2}\right) \| A_{1} T_{v_{2}}^{\Theta_{2}}\left(I-v_{2} A_{2}\right) v_{n} \\
\leq \| T_{v_{2}}^{\Theta_{2}}\left(I-v_{2} A_{2}\right) v_{n}-T_{v_{2}}^{\Theta_{2}}\left(I-v_{2}^{\Theta_{2}}\left(I-v_{2} A_{2}\right) p \|^{2}\right. \\
\leq\left\|v_{n}-p\right\|^{2}+v_{2}\left(v_{2}-2 \zeta_{2}\right)\left\|A_{2} v_{n}-A_{2} p\right\|^{2} \\
\leq\left\|v_{n}-p\right\|^{2},
\end{gathered}
$$

$$
\begin{aligned}
\left\|\Lambda_{n}^{i} u_{n}-p\right\|^{2} \leq & \left\|x_{n}-p\right\|^{2}+\lambda_{i, n}\left(\lambda_{i, n}-2 \eta_{i}\right) \\
& \times\left\|B_{i} \Lambda_{n}^{i-1} u_{n}-B_{i} p\right\|^{2}, \quad i \in\{1,2, \ldots, N\},
\end{aligned}
$$

$$
\begin{aligned}
\| k_{n}- & p \|^{2} \\
\leq & \left(1+\gamma_{n}\right)\left\|z_{n}-p\right\|^{2}+\left(1-\delta_{n}\right) \\
& \times\left(k-\delta_{n}\right)\left\|z_{n}-S^{n} z_{n}\right\|^{2}+c_{n} \\
\leq & \left(1+\gamma_{n}\right)\left\|z_{n}-p\right\|^{2}+c_{n} \\
\leq & \left(1+\gamma_{n}\right)\left(\left\|x_{n}-p\right\|^{2}+s_{n} \frac{\|(\gamma Q-V) p\|^{2}}{\bar{\gamma}-\gamma l}\right)+c_{n}, \\
& \left\|\Lambda_{n}^{i} u_{n}-p\right\|^{2} \\
& \leq\left\|x_{n}-p\right\|^{2}-\left\|\Lambda_{n}^{i-1} u_{n}-\Lambda_{n}^{i} u_{n}\right\|^{2} \\
& +2 \lambda_{i, n}\left\|\Lambda_{n}^{i-1} u_{n}-\Lambda_{n}^{i} u_{n}\right\|\left\|B_{i} \Lambda_{n}^{i-1} u_{n}-B_{i} p\right\| \\
&
\end{aligned}
$$


Utilizing (158) and (168) we obtain

$$
\begin{aligned}
& \left\|x_{n+1}-p\right\|^{2} \\
& \leq \quad\left(1-\alpha_{n}\right)\left\|x_{n}-p\right\|^{2}+\alpha_{n}\left\|k_{n}-p\right\|^{2} \\
& \leq \quad\left(1-\alpha_{n}\right)\left\|x_{n}-p\right\|^{2} \\
& \quad+\alpha_{n}\left[\left(1+\gamma_{n}\right)\left(\left\|x_{n}-p\right\|^{2}+s_{n} \frac{\|(\gamma Q-V) p\|^{2}}{\bar{\gamma}-\gamma l}\right)+c_{n}\right] \\
& \leq \quad\left(1+\gamma_{n}\right)\left(\left\|x_{n}-p\right\|^{2}+s_{n} \frac{\|(\gamma Q-V) p\|^{2}}{\bar{\gamma}-\gamma l}\right)+c_{n} \\
& =\left\|x_{n}-p\right\|^{2}+\gamma_{n}\left\|x_{n}-p\right\|^{2} \\
& \quad+s_{n}\left(1+\gamma_{n}\right) \frac{\|(\gamma Q-V) p\|^{2}}{\bar{\gamma}-\gamma l}+c_{n} .
\end{aligned}
$$

Since $\sum_{n=1}^{\infty} s_{n}<\infty, \sum_{n=1}^{\infty} \gamma_{n}<\infty$ and $\sum_{n=1}^{\infty} c_{n}<\infty$, by Lemma 13 we have that $\lim _{n \rightarrow \infty}\left\|x_{n}-p\right\|$ exists. Thus $\left\{x_{n}\right\}$ is bounded and so are the sequences $\left\{u_{n}\right\},\left\{v_{n}\right\},\left\{z_{n}\right\}$ and $\left\{k_{n}\right\}$.

Also, utilizing Lemmas 10 and 16(b) we obtain from (158), (164), (165), and (168) that

$$
\begin{aligned}
\left\|z_{n}-p\right\|^{2} & \| \beta_{n}\left(x_{n}-p\right)+\left(1-\beta_{n}\right)\left(T_{n} G v_{n}-p\right) \\
& \quad+s_{n}\left(\gamma Q x_{n}-V T_{n} G v_{n}\right) \|^{2} \\
\leq & \left\|\beta_{n}\left(x_{n}-p\right)+\left(1-\beta_{n}\right)\left(T_{n} G v_{n}-p\right)\right\|^{2} \\
& +2 s_{n}\left\langle\left(\gamma Q x_{n}-V T_{n} G v_{n}\right), z_{n}-p\right\rangle \\
= & \beta_{n}\left\|x_{n}-p\right\|^{2}+\left(1-\beta_{n}\right)\left\|T_{n} G v_{n}-p\right\|^{2} \\
& -\beta_{n}\left(1-\beta_{n}\right)\left\|x_{n}-T_{n} G v_{n}\right\|^{2} \\
& +2 s_{n}\left\langle\left(\gamma Q x_{n}-V T_{n} G v_{n}\right), z_{n}-p\right\rangle \\
\leq & \beta_{n}\left\|x_{n}-p\right\|^{2}+\left(1-\beta_{n}\right)\left\|x_{n}-p\right\|^{2} \\
& -\beta_{n}\left(1-\beta_{n}\right)\left\|x_{n}-T_{n} G v_{n}\right\|^{2} \\
& +2 s_{n}\left\|\gamma Q x_{n}-V T_{n} G v_{n}\right\|\left\|z_{n}-p\right\| \\
= & \left\|x_{n}-p\right\|^{2}-\beta_{n}\left(1-\beta_{n}\right)\left\|x_{n}-T T_{n} G v_{n}\right\|^{2} \\
& +2 s_{n}\left\|\gamma Q x_{n}-V T_{n} G v_{n}\right\|\left\|z_{n}-p\right\| \\
\leq & \left\|x_{n}-p\right\|^{2}+2 s_{n}\left\|\gamma Q x_{n}-V T_{n} G v_{n}\right\|\left\|z_{n}-p\right\|,
\end{aligned}
$$

and hence

$$
\begin{aligned}
& \left\|x_{n+1}-p\right\|^{2} \\
& \leq\left(1-\alpha_{n}\right)\left\|x_{n}-p\right\|^{2}+\alpha_{n}\left\|k_{n}-p\right\|^{2} \\
& \leq\left(1-\alpha_{n}\right)\left\|x_{n}-p\right\|^{2}+\alpha_{n}\left[\left(1+\gamma_{n}\right)\left\|z_{n}-p\right\|^{2}+c_{n}\right] \\
& \leq\left(1-\alpha_{n}\right)\left\|x_{n}-p\right\|^{2} \\
& \quad+\alpha_{n}\left[( 1 + \gamma _ { n } ) \left(\left\|x_{n}-p\right\|^{2}-\beta_{n}\left(1-\beta_{n}\right)\left\|x_{n}-T_{n} G v_{n}\right\|^{2}\right.\right. \\
& \left.\left.\quad+2 s_{n}\left\|\gamma Q x_{n}-V T_{n} G v_{n}\right\|\left\|z_{n}-p\right\|\right)+c_{n}\right] \\
& \leq\left(1-\alpha_{n}\right)\left\|x_{n}-p\right\|^{2}+\alpha_{n}\left(1+\gamma_{n}\right) \\
& \quad \times\left(\left\|x_{n}-p\right\|^{2}-\beta_{n}\left(1-\beta_{n}\right)\left\|x_{n}-T_{n} G v_{n}\right\|^{2}\right. \\
& \left.\quad+2 s_{n}\left\|\gamma Q x_{n}-V T_{n} G v_{n}\right\|\left\|z_{n}-p\right\|\right)+c_{n} \\
& \leq\left(1+\gamma_{n}\right)\left\|x_{n}-p\right\|^{2} \\
& \quad-\alpha_{n}\left(1+\gamma_{n}\right) \beta_{n}\left(1-\beta_{n}\right)\left\|x_{n}-T_{n} G v_{n}\right\|^{2} \\
& \quad+2 s_{n}\left(1+\gamma_{n}\right)\left\|\gamma Q x_{n}-V T_{n} G v_{n}\right\|\left\|z_{n}-p\right\|+c_{n} .
\end{aligned}
$$

So, it follows that

$$
\begin{aligned}
& \alpha\left(1+\gamma_{n}\right) a(1-\widehat{a})\left\|x_{n}-T_{n} G v_{n}\right\|^{2} \\
& \leq \alpha_{n}\left(1+\gamma_{n}\right) \beta_{n}\left(1-\beta_{n}\right)\left\|x_{n}-T_{n} G v_{n}\right\|^{2} \\
& \leq\left\|x_{n}-p\right\|^{2}-\left\|x_{n+1}-p\right\|^{2}+\gamma_{n}\left\|x_{n}-p\right\|^{2} \\
&+2 s_{n}\left(1+\gamma_{n}\right)\left\|\gamma Q x_{n}-V T_{n} G v_{n}\right\|\left\|z_{n}-p\right\|+c_{n} .
\end{aligned}
$$

Since $\lim _{n \rightarrow \infty} s_{n}=0, \lim _{n \rightarrow \infty} \gamma_{n}=0$ and $\lim _{n \rightarrow \infty} c_{n}=0$, it follows from the existence of $\lim _{n \rightarrow \infty}\left\|x_{n}-p\right\|$ and the boundedness of $\left\{x_{n}\right\},\left\{v_{n}\right\}$, and $\left\{z_{n}\right\}$ that

$$
\lim _{n \rightarrow \infty}\left\|x_{n}-T_{n} G v_{n}\right\|=0 .
$$

Note that

$$
\begin{aligned}
& \left\|z_{n}-x_{n}\right\| \\
& \quad=\left\|\left(1-\beta_{n}\right)\left(T_{n} G v_{n}-x_{n}\right)+s_{n}\left(\gamma Q x_{n}-V T_{n} G v_{n}\right)\right\| \\
& \quad \leq\left\|T_{n} G v_{n}-x_{n}\right\|+s_{n}\left\|\gamma Q x_{n}-V T_{n} G v_{n}\right\| .
\end{aligned}
$$

Hence, it follows from (171) and $\lim _{n \rightarrow \infty} s_{n}=0$ that

$$
\lim _{n \rightarrow \infty}\left\|x_{n}-z_{n}\right\|=0
$$


In the meantime, from (168) and (171) it follows that

$$
\begin{aligned}
& \left\|x_{n+1}-p\right\|^{2} \\
& \leq\left(1-\alpha_{n}\right)\left\|x_{n}-p\right\|^{2}+\alpha_{n}\left\|k_{n}-p\right\|^{2} \\
& \leq\left(1-\alpha_{n}\right)\left\|x_{n}-p\right\|^{2} \\
& +\alpha_{n}\left[\left(1+\gamma_{n}\right)\left\|z_{n}-p\right\|^{2}+\left(1-\delta_{n}\right)\right. \\
& \left.\times\left(k-\delta_{n}\right)\left\|z_{n}-S^{n} z_{n}\right\|^{2}+c_{n}\right] \\
& \leq\left(1-\alpha_{n}\right)\left\|x_{n}-p\right\|^{2} \\
& +\alpha_{n}\left[( 1 + \gamma _ { n } ) \left(\left\|x_{n}-p\right\|^{2}+2 s_{n}\left\|\gamma Q x_{n}-V T_{n} G v_{n}\right\|\right.\right. \\
& \left.\times\left\|z_{n}-p\right\|\right) \\
& \left.+\left(1-\delta_{n}\right)\left(k-\delta_{n}\right)\left\|z_{n}-S^{n} z_{n}\right\|^{2}+c_{n}\right] \\
& \leq\left(1-\alpha_{n}\right)\left\|x_{n}-p\right\|^{2}+\alpha_{n}\left(1+\gamma_{n}\right)\left\|x_{n}-p\right\|^{2} \\
& +2 s_{n}\left(1+\gamma_{n}\right)\left\|\gamma Q x_{n}-V T_{n} G v_{n}\right\|\left\|z_{n}-p\right\| \\
& +\alpha_{n}\left(1-\delta_{n}\right)\left(k-\delta_{n}\right)\left\|z_{n}-S^{n} z_{n}\right\|^{2}+c_{n} \\
& \leq\left(1+\gamma_{n}\right)\left\|x_{n}-p\right\|^{2} \\
& +2 s_{n}\left(1+\gamma_{n}\right)\left\|\gamma Q x_{n}-V T_{n} G v_{n}\right\|\left\|z_{n}-p\right\| \\
& +\alpha_{n}\left(1-\delta_{n}\right)\left(k-\delta_{n}\right)\left\|z_{n}-S^{n} z_{n}\right\|^{2}+c_{n},
\end{aligned}
$$

which together with $0<k+\epsilon \leq \delta_{n} \leq d<1$ leads to

$$
\begin{aligned}
& \alpha(1-d) \epsilon\left\|z_{n}-S^{n} z_{n}\right\|^{2} \\
& \leq \alpha_{n}\left(1-\delta_{n}\right)\left(\delta_{n}-k\right)\left\|z_{n}-S^{n} z_{n}\right\|^{2} \\
& \leq\left\|x_{n}-p\right\|^{2}-\left\|x_{n+1}-p\right\|^{2}+\gamma_{n}\left\|x_{n}-p\right\|^{2} \\
& +2 s_{n}\left(1+\gamma_{n}\right)\left\|\gamma Q x_{n}-V T_{n} G v_{n}\right\|\left\|z_{n}-p\right\|+c_{n} .
\end{aligned}
$$

Consequently, from $\lim _{n \rightarrow \infty} s_{n}=0, \lim _{n \rightarrow \infty} \gamma_{n}=0$, $\lim _{n \rightarrow \infty} c_{n}=0$, and the existence of $\lim _{n \rightarrow \infty}\left\|x_{n}-p\right\|$, we get

$$
\lim _{n \rightarrow \infty}\left\|z_{n}-S^{n} z_{n}\right\|=0 .
$$

Since $k_{n}-z_{n}=\left(1-\delta_{n}\right)\left(S^{n} z_{n}-z_{n}\right)$, from (179) we have

$$
\lim _{n \rightarrow \infty}\left\|k_{n}-z_{n}\right\|=0 \text {. }
$$

Note that

$$
\left\|x_{n+1}-x_{n}\right\|=\alpha_{n}\left\|k_{n}-x_{n}\right\| \leq\left\|k_{n}-z_{n}\right\|+\left\|z_{n}-x_{n}\right\| .
$$

Hence from (176) and (180) we have

$$
\lim _{n \rightarrow \infty}\left\|x_{n+1}-x_{n}\right\|=0 \text {. }
$$

Repeating the same arguments as those of Step 3 in the proof of Theorem 26, we can obtain that $\left\|x_{n}-u_{n}\right\| \rightarrow 0, \| x_{n}-$ $v_{n}\|\rightarrow 0,\| v_{n}-G v_{n}\|\rightarrow 0,\| v_{n}-P_{C}(I-(2 / L) \nabla f) v_{n} \| \rightarrow 0$, $\left\|z_{n}-S z_{n}\right\| \rightarrow 0$, and $\left\|\Lambda_{n}^{i-1} u_{n}-\Lambda_{n}^{i} u_{n}\right\| \rightarrow 0, i \in\{1,2, \ldots, N\}$ as $n \rightarrow \infty$.

Since $\left\{x_{n}\right\}$ is bounded, there exists a subsequence $\left\{x_{n_{i}}\right\}$ of $\left\{x_{n}\right\}$ which converges weakly to $w$. It is easy to see that $z_{n_{i}} \rightarrow w, u_{n_{i}} \rightarrow w, v_{n_{i}} \rightarrow w$, and $\Lambda_{n_{i}}^{m} u_{n_{i}} \rightarrow w$, where $m \in\{1,2, \ldots, N\}$. Since $S$ is uniformly continuous and $\left\|z_{n}-S z_{n}\right\| \rightarrow 0$ as $n \rightarrow \infty$, we get $\lim _{n \rightarrow \infty} \| z_{n}-$ $S^{m} z_{n} \|=0$ for any $m \geq 1$. Hence from Lemma 24, we obtain $w \in \operatorname{Fix}(S)$. In the meantime, utilizing Lemma 12, we deduce from $v_{n_{i}} \rightarrow w, x_{n_{i}} \rightarrow w$, (118), and (123) that $w \in \operatorname{SGEP}(G)$ and $w \in \operatorname{Fix}\left(P_{C}(I-(2 / L) \nabla f)\right)=V I(C, \nabla f)=\Gamma$. Repeating the same arguments as those of Step 4 in the proof of Theorem 26, we can conclude that $w \in \cap_{m=1}^{N} I\left(B_{m}, R_{m}\right)$ and $w \in \operatorname{GMEP}(\Theta, \varphi, A)$. Therefore, $w \in \operatorname{GMEP}(\Theta, \varphi, A) \cap$ $\operatorname{SGEP}(G) \cap \cap_{i=1}^{N} I\left(B_{i}, R_{i}\right) \cap \operatorname{Fix}(S) \cap \Gamma=: \Omega$. This shows that $\omega_{w}\left(x_{n}\right) \subset \Omega$.

Next let us show that $\omega_{w}\left(x_{n}\right)$ is a single-point set. As a matter of fact, let $\left\{x_{n_{j}}\right\}$ be another subsequence of $\left\{x_{n}\right\}$ such that $x_{n_{j}} \rightarrow w^{\prime}$. Then we get $w^{\prime} \in \Omega$. If $w \neq w^{\prime}$, from the Opial condition, we have

$$
\begin{aligned}
\lim _{n \rightarrow \infty}\left\|x_{n}-w\right\| & =\lim _{i \rightarrow \infty}\left\|x_{n_{i}}-w\right\|<\lim _{i \rightarrow \infty}\left\|x_{n_{i}}-w^{\prime}\right\| \\
& =\lim _{n \rightarrow \infty}\left\|x_{n}-w^{\prime}\right\|=\lim _{j \rightarrow \infty}\left\|x_{n_{j}}-w^{\prime}\right\| \\
& <\lim _{j \rightarrow \infty}\left\|x_{n_{j}}-w\right\|=\lim _{n \rightarrow \infty}\left\|x_{n}-w\right\| .
\end{aligned}
$$

This attains a contradiction. So we have $w=w^{\prime}$. Put $w_{n}=$ $P_{\Omega} x_{n}$. Since $w \in \Omega$, we have $\left\langle x_{n}-w_{n}, w_{n}-w\right\rangle \geq 0$. By Lemma 14, we have that $\left\{w_{n}\right\}$ converges strongly to some $\widetilde{w} \in$ $\Omega$. Since $\left\{x_{n}\right\}$ converges weakly to $w$, we have

$$
\langle w-\widetilde{w}, \widetilde{w}-w\rangle \geq 0 .
$$

Therefore we obtain $w=\widetilde{w}=\lim _{n \rightarrow \infty} P_{\Omega} x_{n}$. This completes the proof.

Corollary 31. Let $C$ be a nonempty closed convex subset of a real Hilbert space $H$. Let $f: C \rightarrow \mathbf{R}$ be a convex functional with L-Lipschitz continuous gradient $\nabla f$. Let $\Theta, \Theta_{1}, \Theta_{2}$ be three bifunctions from $\mathrm{C} \times \mathrm{C}$ to $\mathbf{R}$ satisfying $(\mathrm{H} 1)-(\mathrm{H} 4)$ and $\varphi$ : $C \rightarrow \mathbf{R}$ be a lower semicontinuous and convex functional. Let $R_{i}: C \rightarrow 2^{H}$ be a maximal monotone mapping and let $A, A_{k}: H \rightarrow H$ and $B_{i}: C \rightarrow H$ be $\zeta_{-}$ inverse strongly monotone, $\zeta_{k}$-inverse strongly monotone, and $\eta_{i}$-inverse-strongly monotone, respectively, for $k=1,2$ and $i=$ 1,2 . Let $S: C \rightarrow C$ be a uniformly continuous asymptotically $k$-strict pseudocontractive mapping in the intermediate sense for some $0 \leq k<1$ with sequence $\left\{\gamma_{n}\right\} \subset[0, \infty)$ such that $\sum_{n=1}^{\infty} \gamma_{n}<\infty$ and $\left\{c_{n}\right\} \subset[0, \infty)$ such that $\sum_{n=1}^{\infty} c_{n}<\infty$. Let $V$ be a $\bar{\gamma}$-strongly positive bounded linear operator and $Q: H \rightarrow$ $H$ an l-Lipschitzian mapping with $\gamma l<\bar{\gamma}$. Assume that $\Omega:=$ $\operatorname{GMEP}(\Theta, \varphi, A) \cap \operatorname{SGEP}(G) \cap I\left(B_{1}, R_{1}\right) \cap I\left(B_{2}, R_{2}\right) \cap$ Fix $(S) \cap \Gamma$ is nonempty where $G$ is defined as in Proposition CY. Let $\left\{r_{n}\right\}$ be a sequence in $[0,2 \zeta]$ and $\left\{\alpha_{n}\right\},\left\{\beta_{n}\right\}$, and $\left\{\delta_{n}\right\}$ sequences in 
$[0,1]$ such that $0<\alpha \leq \alpha_{n} \leq 1$ and $0<k+\epsilon \leq \delta_{n} \leq d<1$, and let $\nu_{k} \in\left(0,2 \zeta_{k}\right), k=1,2$ and $\left\{\lambda_{i, n}\right\} \subset\left[a_{i}, b_{i}\right] \subset\left(0,2 \eta_{i}\right)$, $i=1,2$. Pick any $x_{1} \in H$ and let $\left\{x_{n}\right\}$ be a sequence generated by the following algorithm:

$$
\begin{aligned}
u_{n} & =S_{r_{n}}^{(\Theta, \varphi)}\left(I-r_{n} A\right) x_{n}, \\
v_{n} & =J_{R_{2}, \lambda_{2, n}}\left(I-\lambda_{2, n} B_{2}\right) J_{R_{1}, \lambda_{1, n}}\left(I-\lambda_{1, n} B_{1}\right) u_{n}, \\
z_{n} & =s_{n} \gamma Q x_{n}+\beta_{n} x_{n}+\left(\left(1-\beta_{n}\right) I-s_{n} V\right) T_{n} G v_{n}, \\
k_{n} & =\delta_{n} z_{n}+\left(1-\delta_{n}\right) S^{n} z_{n}, \\
x_{n+1} & =\left(1-\alpha_{n}\right) x_{n}+\alpha_{n} k_{n}, \quad \forall n \geq 1,
\end{aligned}
$$

where $P_{C}\left(I-\lambda_{n} \nabla f\right)=s_{n} I+\left(1-s_{n}\right) T_{n}$ (here $T_{n}$ is nonexpansive, $s_{n}=\left(2-\lambda_{n} L\right) / 4 \in(0,1 / 2)$ for each $\left.\lambda_{n} \in(0,2 / L)\right)$. Assume that the following conditions are satisfied:

(i) $K: H \rightarrow \mathbf{R}$ is strongly convex with constant $\sigma>0$ and its derivative $K^{\prime}$ is Lipschitz continuous with constant $v>0$ such that the function $x \mapsto\left\langle y-x, K^{\prime}(x)\right\rangle$ is weakly upper semicontinuous for each $y \in H$;

(ii) for each $x \in H$, there exist a bounded subset $D_{x} \subset C$ and $z_{x} \in C$ such that for any $y \notin D_{x}$,

$$
\begin{aligned}
& \Theta\left(y, z_{x}\right)+\varphi\left(z_{x}\right)-\varphi(y) \\
& +\frac{1}{r}\left\langle K^{\prime}(y)-K^{\prime}(x), z_{x}-y\right\rangle<0 ;
\end{aligned}
$$

(iii) $s_{n} \in(0,1 / 2)$ for each $\lambda_{n} \in(0,2 / L)$, and $\sum_{n=1}^{\infty} s_{n}<$ $\infty\left(\Leftrightarrow \sum_{n=1}^{\infty}\left((2 / L)-\lambda_{n}\right)<\infty\right) ;$

(iv) $0<\liminf _{n \rightarrow \infty} \beta_{n} \leq \limsup _{n \rightarrow \infty} \beta_{n}<1$ and $0<$ $\liminf _{n \rightarrow \infty} r_{n} \leq \limsup _{n \rightarrow \infty} r_{n}<2 \zeta$.

Then $\left\{x_{n}\right\}$ converges weakly to $w=\lim _{n \rightarrow \infty} P_{\Omega} x_{n}$ provided that $S_{r}^{(\Theta, \varphi)}$ is firmly nonexpansive.

Corollary 32. Let $C$ be a nonempty closed convex subset of a real Hilbert space $H$. Let $f: C \rightarrow \mathbf{R}$ be a convex functional with L-Lipschitz continuous gradient $\nabla f$. Let $\Theta, \Theta_{1}, \Theta_{2}$ be three bifunctions from $C \times C$ to $\mathbf{R}$ satisfying $(\mathrm{H} 1)-(\mathrm{H} 4)$ and $\varphi$ : $C \rightarrow \mathbf{R}$ a lower semicontinuous and convex functional. Let $R: C \rightarrow 2^{H}$ be a maximal monotone mapping and let $A, A_{k}$ : $H \rightarrow H$ and $B: C \rightarrow H$ be $\zeta$-inverse strongly monotone, $\zeta_{k^{-}}$ inverse strongly monotone, and $\xi$-inverse-strongly monotone, respectively, for $k=1,2$. Let $S: C \rightarrow C$ be a uniformly continuous asymptotically $k$-strict pseudocontractive mapping in the intermediate sense for some $0 \leq k<1$ with sequence $\left\{\gamma_{n}\right\} \subset[0, \infty)$ such that $\sum_{n=1}^{\infty} \gamma_{n}<\infty$ and $\left\{c_{n}\right\} \subset[0, \infty)$ such that $\sum_{n=1}^{\infty} c_{n}<\infty$. Let $V$ be a $\bar{\gamma}$-strongly positive bounded linear operator and $Q: H \rightarrow H$ an l-Lipschitzian mapping with $\gamma l<\bar{\gamma}$. Assume that $\Omega:=\operatorname{GMEP}(\Theta, \varphi, A) \cap \operatorname{SGEP}(G) \cap$ $I(B, R) \cap \operatorname{Fix}(S) \cap \Gamma$ is nonempty, where $G$ is defined as in Proposition CY. Let $\left\{r_{n}\right\}$ be a sequence in $[0,2 \zeta]$ and $\left\{\alpha_{n}\right\},\left\{\beta_{n}\right\}$, and $\left\{\delta_{n}\right\}$ sequences in $[0,1]$ such that $0<\alpha \leq \alpha_{n} \leq 1$ and $0<k+\epsilon \leq \delta_{n} \leq d<1$, and let $\nu_{k} \in\left(0,2 \zeta_{k}\right), k=1,2$ and $\left\{\rho_{n}\right\} \subset[a, b] \subset(0,2 \xi)$. Pick any $x_{1} \in H$ and let $\left\{x_{n}\right\}$ be a sequence generated by the following algorithm:

$$
\begin{aligned}
u_{n} & =S_{r_{n}}^{(\Theta, \varphi)}\left(I-r_{n} A\right) x_{n}, \\
v_{n} & =J_{R, \rho_{n}}\left(I-\rho_{n} B\right) u_{n}, \\
z_{n} & =s_{n} \gamma Q x_{n}+\beta_{n} x_{n}+\left(\left(1-\beta_{n}\right) I-s_{n} V\right) T_{n} G v_{n}, \\
k_{n} & =\delta_{n} z_{n}+\left(1-\delta_{n}\right) S^{n} z_{n}, \\
x_{n+1} & =\left(1-\alpha_{n}\right) x_{n}+\alpha_{n} k_{n}, \quad \forall n \geq 1,
\end{aligned}
$$

where $P_{C}\left(I-\lambda_{n} \nabla f\right)=s_{n} I+\left(1-s_{n}\right) T_{n}$ (here $T_{n}$ is nonexpansive, $s_{n}=\left(2-\lambda_{n} L\right) / 4 \in(0,1 / 2)$ for each $\left.\lambda_{n} \in(0,2 / L)\right)$. Assume that the following conditions are satisfied:

(i) $K: H \rightarrow \mathbf{R}$ is strongly convex with constant $\sigma>0$ and its derivative $K^{\prime}$ is Lipschitz continuous with constant $v>0$ such that the function $x \mapsto\left\langle y-x, K^{\prime}(x)\right\rangle$ is weakly upper semicontinuous for each $y \in H$;

(ii) for each $x \in H$, there exist a bounded subset $D_{x} \subset C$ and $z_{x} \in C$ such that for any $y \notin D_{x}$,

$$
\begin{aligned}
& \Theta\left(y, z_{x}\right)+\varphi\left(z_{x}\right)-\varphi(y) \\
& +\frac{1}{r}\left\langle K^{\prime}(y)-K^{\prime}(x), z_{x}-y\right\rangle<0
\end{aligned}
$$

(iii) $s_{n} \in(0,1 / 2)$ for each $\lambda_{n} \in(0,2 / L)$, and $\sum_{n=1}^{\infty} s_{n}<$ $\infty\left(\Leftrightarrow \sum_{n=1}^{\infty}\left((2 / L)-\lambda_{n}\right)<\infty\right)$;

(iv) $0<\liminf _{n \rightarrow \infty} \beta_{n} \leq \limsup _{n \rightarrow \infty} \beta_{n}<1$ and $0<$ $\liminf _{n \rightarrow \infty} r_{n} \leq \lim \sup _{n \rightarrow \infty} r_{n}<2 \zeta$.

Then $\left\{x_{n}\right\}$ converges weakly to $w=\lim _{n \rightarrow \infty} P_{\Omega} x_{n}$ provided that $S_{r}^{(\Theta, \varphi)}$ is firmly nonexpansive.

Corollary 33. Let $C$ be a nonempty closed convex subset of a real Hilbert space $H$. Let $f: C \rightarrow \mathbf{R}$ be a convex functional with L-Lipschitz continuous gradient $\nabla f$. Let $\Theta, \Theta_{1}, \Theta_{2}$ be three bifunctions from $\mathrm{C} \times \mathrm{C}$ to $\mathbf{R}$ satisfying $(\mathrm{H1})-(\mathrm{H} 4)$ and $\varphi$ : $C \rightarrow \mathbf{R}$ a lower semicontinuous and convex functional. Let $R: C \rightarrow 2^{H}$ be a maximal monotone mapping and let $A, A_{k}$ : $H \rightarrow H$ and $B: C \rightarrow H$ be $\zeta$-inverse strongly monotone, $\zeta_{k^{-}}$ inverse strongly monotone, and $\xi$-inverse-strongly monotone, respectively, for $k=1,2$. Let $S: C \rightarrow C$ be a uniformly continuous asymptotically $k$-strict pseudocontractive mapping for some $0 \leq k<1$ with sequence $\left\{\gamma_{n}\right\} \subset[0, \infty)$ such that $\sum_{n=1}^{\infty} \gamma_{n}<\infty$. Let $V$ be a $\bar{\gamma}$-strongly positive bounded linear operator and $Q: H \rightarrow H$ be an l-Lipschitzian mapping with $\gamma l<\bar{\gamma}$. Assume that $\Omega:=\operatorname{GMEP}(\Theta, \varphi, A) \cap \operatorname{SGEP}(G) \cap$ $I(B, R) \cap \operatorname{Fix}(S) \cap \Gamma$ is nonempty, where $G$ is defined as in Proposition CY. Let $\left\{r_{n}\right\}$ be a sequence in $[0,2 \zeta]$ and $\left\{\alpha_{n}\right\},\left\{\beta_{n}\right\}$, and $\left\{\delta_{n}\right\}$ sequences in $[0,1]$ such that $0<\alpha \leq \alpha_{n} \leq 1$ and $0<k+\epsilon \leq \delta_{n} \leq d<1$, and let $\nu_{k} \in\left(0,2 \zeta_{k}\right), k=1,2$ and 
$\left\{\rho_{n}\right\} \subset[a, b] \subset(0,2 \xi)$. Pick any $x_{1} \in H$ and let $\left\{x_{n}\right\}$ be a sequence generated by the following algorithm:

$$
\begin{aligned}
u_{n} & =S_{r_{n}}^{(\Theta, \varphi)}\left(I-r_{n} A\right) x_{n}, \\
v_{n} & =J_{R, \rho_{n}}\left(I-\rho_{n} B\right) u_{n}, \\
z_{n} & =s_{n} \gamma Q x_{n}+\beta_{n} x_{n}+\left(\left(1-\beta_{n}\right) I-s_{n} V\right) T_{n} G v_{n}, \\
k_{n} & =\delta_{n} z_{n}+\left(1-\delta_{n}\right) S^{n} z_{n}, \\
x_{n+1} & =\left(1-\alpha_{n}\right) x_{n}+\alpha_{n} k_{n}, \quad \forall n \geq 1,
\end{aligned}
$$

where $P_{C}\left(I-\lambda_{n} \nabla f\right)=s_{n} I+\left(1-s_{n}\right) T_{n}$ (here $T_{n}$ is nonexpansive, $s_{n}=\left(2-\lambda_{n} L\right) / 4 \in(0,1 / 2)$ for each $\left.\lambda_{n} \in(0,2 / L)\right)$. Assume that the following conditions are satisfied:

(i) $K: H \rightarrow \mathbf{R}$ is strongly convex with constant $\sigma>0$ and its derivative $K^{\prime}$ is Lipschitz continuous with constant $\nu>0$ such that the function $x \mapsto\left\langle y-x, K^{\prime}(x)\right\rangle$ is weakly upper semicontinuous for each $y \in H$;

(ii) for each $x \in H$, there exist a bounded subset $D_{x} \subset C$ and $z_{x} \in C$ such that for any $y \notin D_{x}$,

$$
\begin{aligned}
& \Theta\left(y, z_{x}\right)+\varphi\left(z_{x}\right)-\varphi(y) \\
& +\frac{1}{r}\left\langle K^{\prime}(y)-K^{\prime}(x), z_{x}-y\right\rangle<0 ;
\end{aligned}
$$

(iii) $s_{n} \in(0,1 / 2)$ for each $\lambda_{n} \in(0,2 / L)$, and $\sum_{n=1}^{\infty} s_{n}<$ $\infty\left(\Leftrightarrow \sum_{n=1}^{\infty}\left((2 / L)-\lambda_{n}\right)<\infty\right) ;$

(iv) $0<\liminf _{n \rightarrow \infty} \beta_{n} \leq \limsup _{n \rightarrow \infty} \beta_{n}<1$ and $0<$ $\liminf _{n \rightarrow \infty} r_{n} \leq \limsup _{n \rightarrow \infty} r_{n}<2 \zeta$.

Then $\left\{x_{n}\right\}$ converges weakly to $w=\lim _{n \rightarrow \infty} P_{\Omega} x_{n}$ provided that $S_{r}^{(\Theta, \varphi)}$ is firmly nonexpansive.

\section{Conflict of Interests}

The authors declare that there is no conflict of interests regarding the publication of this paper.

\section{Acknowledgments}

This research was partially supported by the National Science Foundation of China (11071169), Innovation Program of Shanghai Municipal Education Commission (09ZZ133), and Ph.D. Program Foundation of Ministry of Education of China (20123127110002). This work was supported partly by the National Science Council of the Republic of China.

\section{References}

[1] J.-L. Lions, Quelques Méthodes de Résolution des Problèmes aux Limites Non Linéaires, Dunod, Paris, France, 1969.

[2] J.-W. Peng and J.-C. Yao, "A new hybrid-extragradient method for generalized mixed equilibrium problems, fixed point problems and variational inequality problems," Taiwanese Journal of Mathematics, vol. 12, no. 6, pp. 1401-1432, 2008.
[3] S. Takahashi and W. Takahashi, "Strong convergence theorem for a generalized equilibrium problem and a nonexpansive mapping in a Hilbert space," Nonlinear Analysis: Theory, Methods \& Applications, vol. 69, no. 3, pp. 1025-1033, 2008.

[4] L.-C. Ceng and J.-C. Yao, "A hybrid iterative scheme for mixed equilibrium problems and fixed point problems," Journal of Computational and Applied Mathematics, vol. 214, no. 1, pp. 186201, 2008.

[5] V. Colao, G. Marino, and H.-K. Xu, "An iterative method for finding common solutions of equilibrium and fixed point problems," Journal of Mathematical Analysis and Applications, vol. 344, no. 1, pp. 340-352, 2008.

[6] L. C. Ceng, A. Petruşel, and J. C. Yao, "Iterative approaches to solving equilibrium problems and fixed point problems of infinitely many nonexpansive mappings," Journal of Optimization Theory and Applications, vol. 143, no. 1, pp. 37-58, 2009.

[7] L.-C. Ceng and J.-C. Yao, "A relaxed extragradient-like method for a generalized mixed equilibrium problem, a general system of generalized equilibria and a fixed point problem," Nonlinear Analysis: Theory, Methods \& Applications, vol. 72, no. 3-4, pp. 1922-1937, 2010.

[8] L.-C. Ceng, C.-Y. Wang, and J.-C. Yao, "Strong convergence theorems by a relaxed extragradient method for a general system of variational inequalities," Mathematical Methods of Operations Research, vol. 67, no. 3, pp. 375-390, 2008.

[9] L.-C. Ceng, Q. H. Ansari, and S. Schaible, "Hybrid extragradient-like methods for generalized mixed equilibrium problems, systems of generalized equilibrium problems and optimization problems," Journal of Global Optimization, vol. 53, no. 1, pp. 69-96, 2012.

[10] L.-C. Ceng, Q. H. Ansari, and J.-C. Yao, "Some iterative methods for finding fixed points and for solving constrained convex minimization problems," Nonlinear Analysis: Theory, Methods \& Applications, vol. 74, no. 16, pp. 5286-5302, 2011.

[11] S. Al-Homidan and L. C. Ceng, "Algorithms of common solutions for generalized mixed equilibria, variational inclusions, and constrained convex minimization," Abstract and Applied Analysis, vol. 2014, Article ID 132053, 25 pages, 2014.

[12] L.-C. Ceng and C.-F. Wen, "Hybrid steepest-descent methods for triple hierarchical variational inequalities," Taiwanese Journal of Mathematics, vol. 17, no. 4, pp. 1441-1472, 2013.

[13] L.-C. Ceng, Q. H. Ansari, and J.-C. Yao, "Relaxed extragradient methods for finding minimum-norm solutions of the split feasibility problem," Nonlinear Analysis: Theory, Methods \& Applications, vol. 75, no. 4, pp. 2116-2125, 2012.

[14] T.-H. Kim and H.-K. Xu, "Convergence of the modified Mann's iteration method for asymptotically strict pseudo-contractions," Nonlinear Analysis: Theory, Methods \& Applications, vol. 68, no. 9, pp. 2828-2836, 2008.

[15] D. R. Sahu, H.-K. Xu, and J.-C. Yao, "Asymptotically strict pseudocontractive mappings in the intermediate sense," Nonlinear Analysis: Theory, Methods \& Applications, vol. 70, no. 10, pp. 3502-3511, 2009.

[16] H.-K. Xu, "Averaged mappings and the gradient-projection algorithm," Journal of Optimization Theory and Applications, vol. 150, no. 2, pp. 360-378, 2011.

[17] K. Geobel and W. A. Kirk, Topics on Metric Fixed-Point Theory, Cambridge University Press, Cambridge, UK, 1990.

[18] M. O. Osilike, S. C. Aniagbosor, and B. G. Akuchu, "Fixed points of asymptotically demicontractive mappings in arbitrary Banach spaces," Panamerican Mathematical Journal, vol. 12, no. 2, pp. 77-88, 2002. 
[19] S. Huang, "Hybrid extragradient methods for asymptotically strict pseudo-contractions in the intermediate sense and variational inequality problems," Optimization, vol. 60, no. 6, pp. 739-754, 2011.

[20] C. Martinez-Yanes and H.-K. Xu, "Strong convergence of the CQ method for fixed point iteration processes," Nonlinear Analysis: Theory, Methods \& Applications, vol. 64, no. 11, pp. 2400-2411, 2006.

[21] N.-J. Huang, "A new completely general class of variational inclusions with noncompact valued mappings," Computers \& Mathematics with Applications, vol. 35, no. 10, pp. 9-14, 1998.

[22] L.-C. Zeng, S.-M. Guu, and J.-C. Yao, "Characterization of $H$-monotone operators with applications to variational inclusions," Computers \& Mathematics with Applications, vol. 50, no. 3-4, pp. 329-337, 2005.

[23] L.-C. Ceng, Q. H. Ansari, M. M. Wong, and J.-C. Yao, "Mann type hybrid extragradient method for variational inequalities, variational inclusions and fixed point problems," Fixed Point Theory, vol. 13, no. 2, pp. 403-422, 2012.

[24] R. T. Rockafellar, "Monotone operators and the proximal point algorithm," SIAM Journal on Control and Optimization, vol. 14, no. 5, pp. 877-898, 1976. 


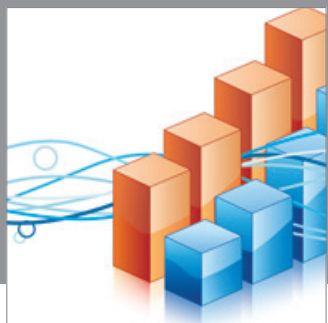

Advances in

Operations Research

mansans

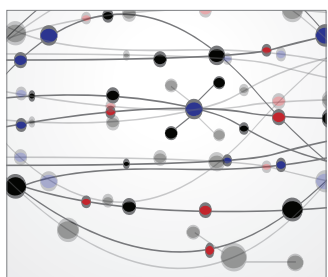

The Scientific World Journal
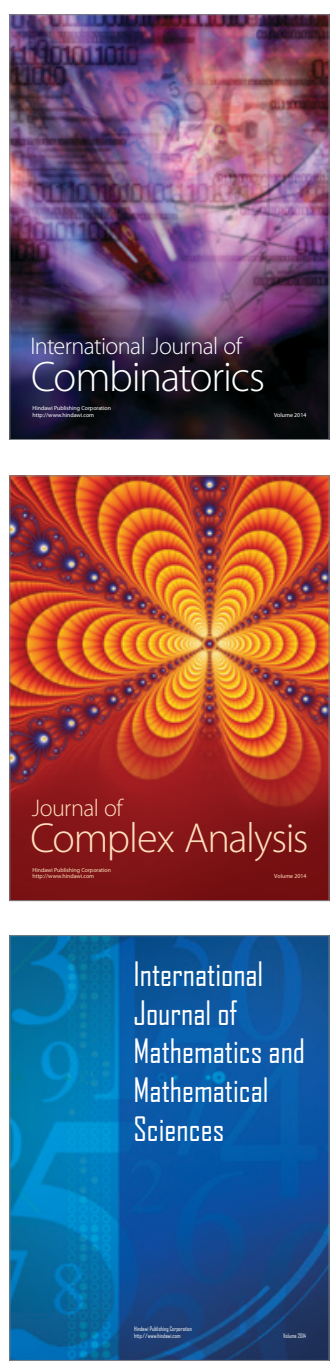
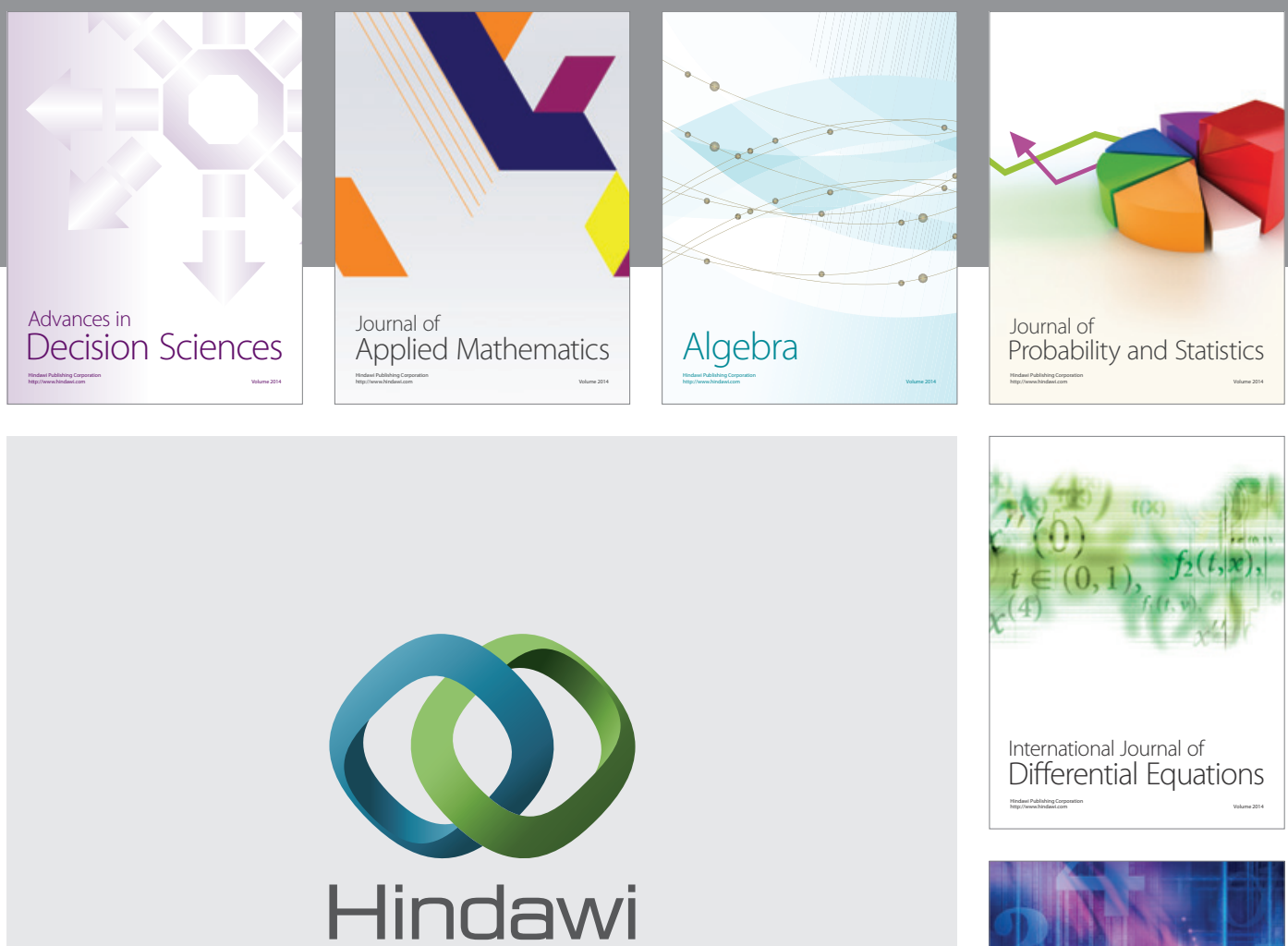

Submit your manuscripts at http://www.hindawi.com
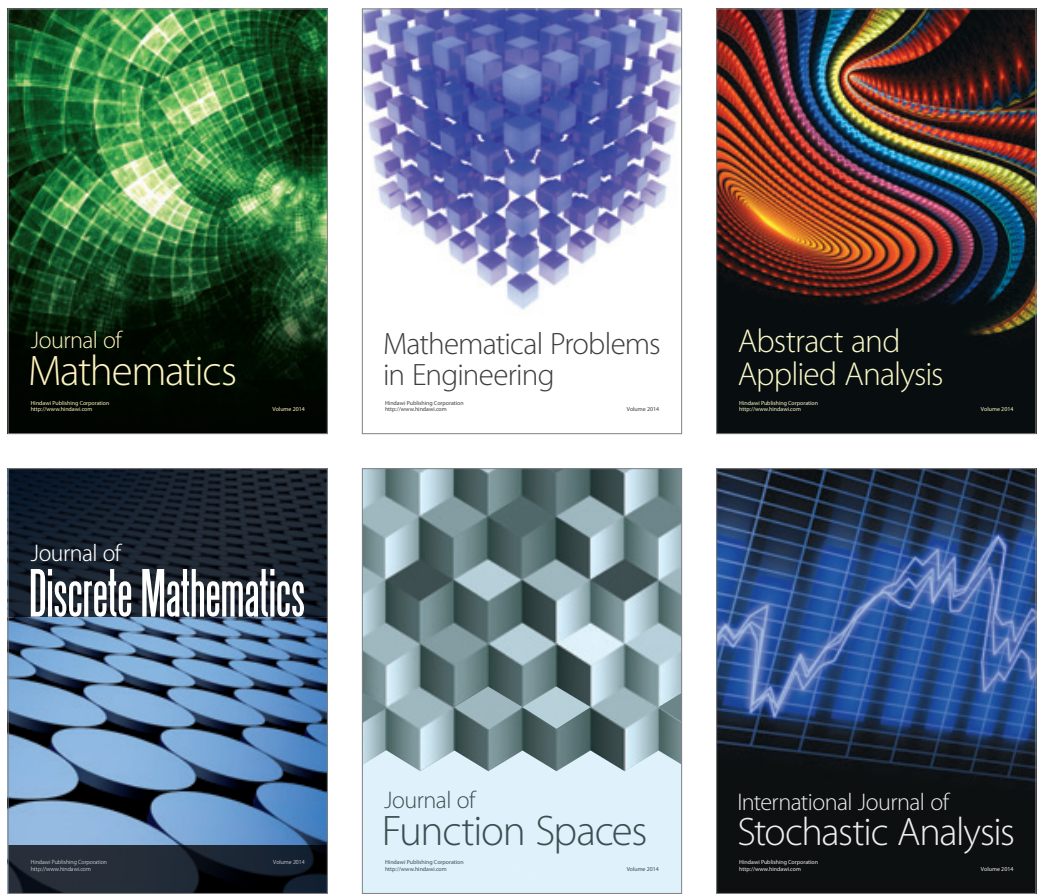

Journal of

Function Spaces

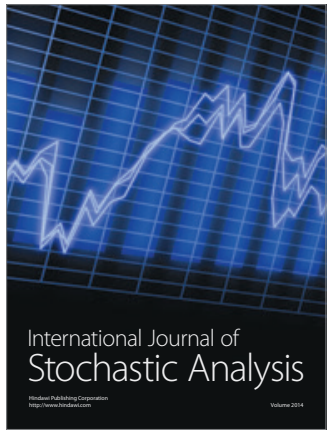

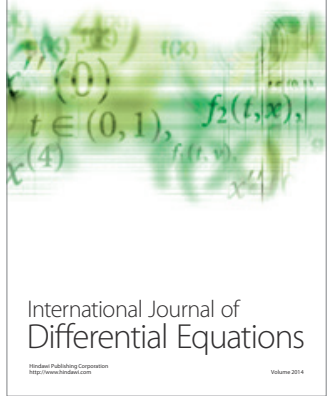
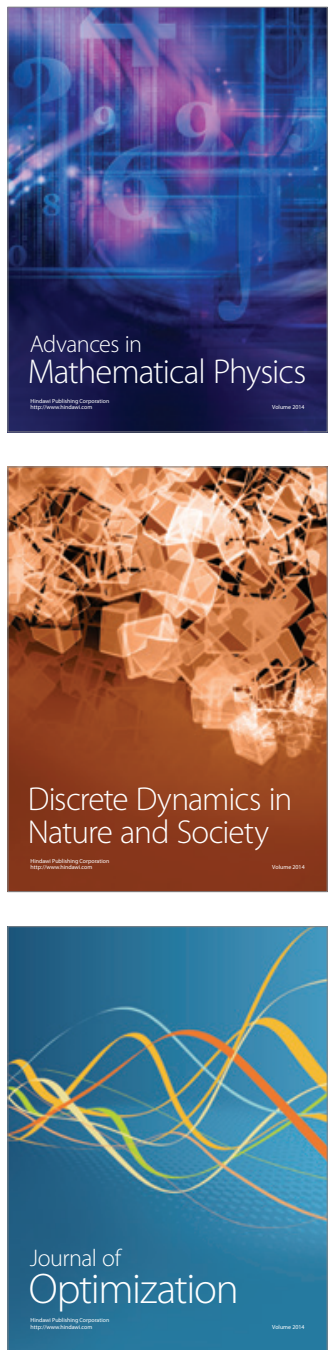